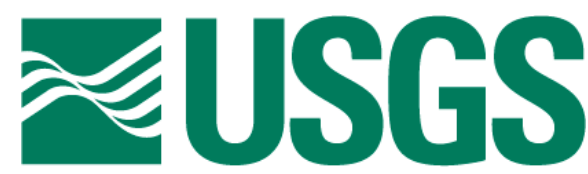

science for a changing world

\title{
USGS environmental characterization of flood sediments left in the New Orleans area after Hurricanes Katrina and Rita, 2005-Progress Report
}

By Geoffrey S. Plumlee, Gregory P. Meeker, John K. Lovelace, Robert J. Rosenbauer, Paul J. Lamothe, Edward T. Furlong, and Charles R. Demas

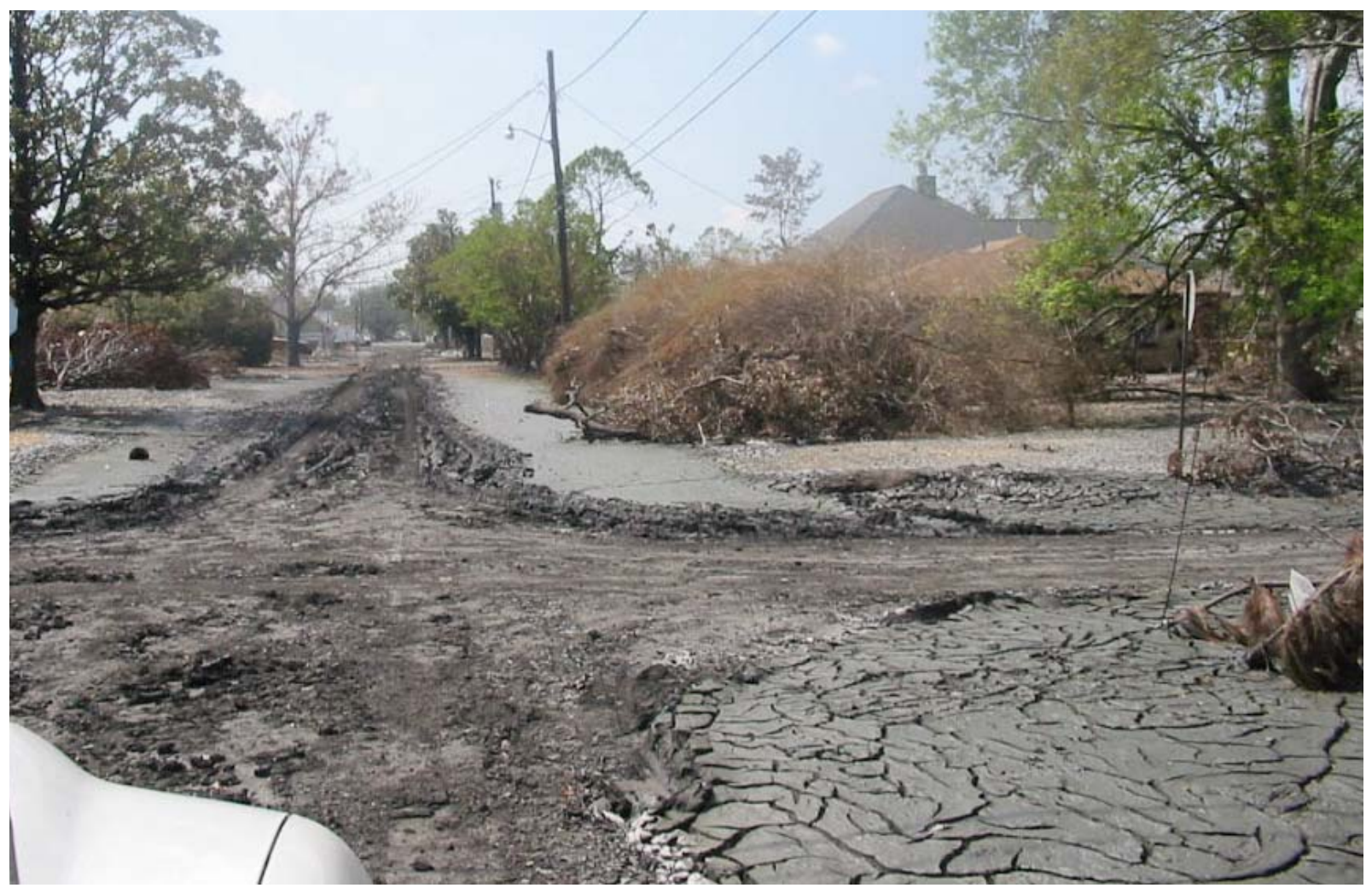

Open-File Report 2006-1023

U.S. Department of the Interior

U.S. Geological Survey 


\section{U.S. Department of the Interior \\ Gale A. Norton, Secretary \\ U.S. Geological Survey \\ P. Patrick Leahy, Acting Director}

U.S. Geological Survey, Reston, Virginia 2006

For product and ordering information:

World Wide Web: http://www.usgs.gov/pubprod

Telephone: 1-888-ASK-USGS

For more information on the USGS-the Federal source for science about the Earth, its natural and living resources, natural hazards, and the environment: World Wide Web: http://www.usgs.gov

Telephone: 1-888-ASK-USGS

Suggested citation:

Plumlee, G.S., Meeker, G.P., Lovelace, J.K., Rosenbauer, R., Lamothe, P.J., Furlong, E.T., and Demas, C.R., 2006, USGS environmental characterization of flood sediments left in the New Orleans area after Hurricanes Katrina and Rita, 2005-Progress Report: U.S. Geologic al Survey Open-File Report 2006-1023, 74 p.

Any use of trade, product, or firm names is for descriptive purposes only and does not imply endorsement by the U.S. Government.

Although this report is in the public domain, permission must be secured from the individual copyright owners to reproduce any copyrighted material contained within this report. 


\section{Overview}

The flooding in the greater New Orleans area that resulted from Hurricanes Katrina and Rita in September, 2005, left behind accumulations of sediments up to many centimeters thick on streets, lawns, parking lots, and other flat surfaces. These flood sediment deposits have been the focus of extensive study by the US Environmental Protection Agency (EPA) and Louisiana Department of Environmental Quality (LDEQ) due to concerns that the sediments may contain elevated levels of heavy metals, organic contaminants, and microbes. Results of the EPA/LDEQ) sampling are presented at http://www.epa.gov/katrina/testresults/sediments/index.html\#2.

The U.S. Geological Survey (USGS) is characterizing a limited number of flood sediment samples that were collected on September 15-16 and October 6-7, 2005, from the greater New Orleans area by personnel from the USGS Louisiana Water Science Center in Baton Rouge. Small samples ( $<3$ pints each) of wet to dry flood sediment were collected from 11 localities around downtown New Orleans on September 15, 2005, and two large samples (40 pints each) of wet flood sediment were collected from the Chalmette area on September 16. Twelve additional samples (8-10 pints each) were collected from New Orleans, Slidell, Rigolets, and Violet (near St. Bernard High School) on October 6 and 7. Sample locations are shown in figure 1 and described in table 1.

A wide variety of physical, inorganic, organic, and microbial characterization methods are being applied to the samples (table 2), with appropriate splits of the samples sent to USGS analytical facilities in Denver, Colorado, Reston, Virginia, St. Petersburg, Florida, Columbia, Missouri, and Menlo Park, California. Additional characterization studies are being performed on splits of the same samples by collaborators at the Colorado School of Mines (Golden, Colorado), EPA National Enforcement Investigations Center (Denver), University of California -Davis, and SUNY Stony Brook, New York. Figures are presented after the main text of the document. Sampling methods are described in Appendix A following the figures. Tables listing locations, types of analyses, and analytical results available to date are included at the end of this document.

The USGS characterization studies of these flood sediments are designed to produce data and interpretations regarding how the sediments and any contained contaminants may respond to environmental processes. This information will be of use to cleanup managers and DoI/USGS scientists assessing environmental impacts of the hurricanes and subsequent cleanup activities. The USGS flood sediment studies focus on:

- The extent to which and the forms in which a wide variety of potential organic and heavy metal contaminants occur in the sediments,

- Mechanisms by which any contaminants present may be released into the environment (either in place or from disposal areas),

- Whether or not the contaminants may be available for uptake by aquatic and terrestrial organisms, and

- How microbial communities may be influenced by chemical and physical properties of the sediments.

The USGS flood sediment study is also addressing several other objectives:

- The flood sediment study is being coordinated with another USGS study examining 
suspended and bottom sediments in Lake Pontchartrain produced by flood waters pumped from New Orleans (study coordinated by Pete VanMetre,

pvanmetre@usgs.gov). The flood sediment characterization results will assist in the discrimination of sediments deposited in Lake Pontchartrain by the floodwater pumping from sediments present prior to pumping.

- USGS flood sediment study results are being used to help calibrate measurements made by an AVIRIS (Airborne Visible and Infra-Red Imaging Spectrometer) remote sensing flight that was flown by NASA over New Orleans early in September, 2005.

- The study results will also be used to test and assist in development of techniques to remotely identify and map organic contaminants in sediments using reflectance spectroscopy and AVIRIS remote sensing.

This study is not intended to provide a detailed hazardous materials-focused assessment of contaminants present in the sediments from throughout the New Orleans area; such an assessment is the appropriate role of EPA and State environmental agencies and not the USGS. A summary of key findings is presented in the following section.

\section{Key Findings}

The Chalmette area samples are composed largely of marine mud, whereas the downtown New Orleans samples analyzed to date have greater proportions of plant matter, sand, small gravel, and anthropogenic debris

Scanning electron microscopy (SEM) and X-ray diffraction studies (tables 3a-3c) indicate the Chalmette samples are composed primarily of marine muds, with very finegrained ( $<20$ micron) particles of quartz, clays, feldspars, silica tests (skeletons) of marine plankton, and pyrite (iron disulfide) (figs. 2 and 3). The pyrite is often framboidal, with raspberry-shaped clusters of $<1$ micron pyrite crystals (Figure 3 ). The Chalmette samples that were collected wet had a consistency similar to cake frosting. However, the mud dries to a very hard material that takes considerable effort to break apart.

Downtown New Orleans samples analyzed to date contain some mud with clays, silica tests, and calcium carbonate (fig. 4). However, they also contain more sand- to fine gravel-sized particles than the Chalmette samples. In addition, the downtown New Orleans samples have more abundant particles of urban construction and commercial materials such as soda lime glass, glass fibers, concrete, nails, and glass jewelry beads. The downtown samples, when dried, tend to occur in large 50-100 micron clumps of smaller particles less than 10-20 microns (fig. 4).

There are generally low concentrations of heavy metals in the Chalmette sediments, but there are elevated concentrations of some metals in a number of the downtown New Orleans flood sediment samples.

Results of the USGS inorganic chemical analyses (table 4) performed to date indicate that the Chalmette 1 and 2 sediments have concentrations of heavy metals (lead, arsenic, chromium, cadmium, mercury, zinc, arsenic) that are somewhat elevated compared to concentrations in average US soils, but are low compared to concentrations of concern in commonly cited soil quality criteria (such as Netherlands soil quality criteria, 1997).

In contrast, the downtown New Orleans samples typically have substantially higher 
levels of metals such as lead, zinc, mercury, copper, arsenic, and cadmium than the Chalmette samples. For many of these elements, concentrations at some sites can exceed the Netherlands and LDEQ soil quality criteria. Lead concentrations, for example, range from 95 to 2160 parts per million (ppm) in the downtown samples, with most having levels above $500 \mathrm{ppm}$. Scanning electron microscopy (SEM) analyses of samples from downtown New Orleans have identified relatively common microscopic (generally less than 10-20 micron) zinc-sulfate-carbonate, lead-phosphate-chlorine-rich, and metallic lead or lead-oxide particles (fig. 5).

\section{Metal enrichments in the downtown New Orleans flood sediments may result in large part from incorporation of pre-Katrina urban soils}

A map of lead variations measured in this study in the Katrina sediments is compared in figure 6 to a map of lead concentrations in New Orleans urban soils determined in the 1990's by Mielke (1999) and Mielke and others (2004). The similarities in spatial patterns of lead enrichments between the Katrina sediment samples and the urban New Orleans soil samples suggest that the Katrina flood sediments in the downtown New Orleans area may be composed, at least in part, of reworked local soil material having previously elevated metal concentrations. A comparison of the chemical, mineralogical, and lead isotopic composition of the flood sediments with archived soil samples collected for the Mielke studies would help assess the validity of this hypothesis.

The USGS analytical results typically are higher in many metals than EPA results for nearby samples, which is most likely due primarily to differences in the methods by which the samples were digested prior to chemical analysis

For most flood sediment samples, the USGS bulk chemical analyses yield higher concentrations of many elements and metals than EPA results for samples collected nearby; this is particularly true for major silicate-forming elements such as aluminum and silicon, but is also commonly the case with metals such as lead. While these differences may in part be due to local spatial variability in the composition of the flood sediments, they likely are due in large part to differences between the USGS and EPA methods used to digest the samples prior to chemical analysis. The USGS method uses a mix of four acids, including HF to break apart silicate minerals. Although the digestion method is not specified on the EPA web site, it most likely is EPA Method 3020, which only uses an aqua regia (two acid) leach that typically does not fully dissolve some aluminosilicate or resistate minerals. Therefore, the elements tied up in these minerals would not contribute to the resulting chemical analyses.

Chemical leach tests using simulated gastric fluids as the leaching fluid indicate that some of the heavy metals contained within the downtown New Orleans flood sediment samples can be quite bioacccessible

Simulated gastric-fluid leach tests (table 5, fig. 7) of the downtown New Orleans samples generally leached relatively high proportions of lead, zinc, cobalt, cadmium, manganese, and arsenic from the solids, indicating that these metals could potentially be bioaccessible if the flood sediments were ingested. These results can be used to help identify metals that might appropriately be evaluated as part of biomonitoring studies of emergency responders, cleanup workers, and residents (especially small children) who 
have reoccupied areas near downtown New Orleans where flood sediments remain or where pre-existing metal rich soils are exposed.

\section{Simulated rain water and landfill water leach tests did not extract high levels of metals from the wet Chalmette sediments}

These leach tests (table 6) have so far been performed on wet sediment samples as received from the field. These tests are designed to evaluate release of metals from the sediments into simulated rain water (USGS test modified from EPA method 1312), simulated landfill leachate (EPA method 1311, performed by the EPA National Enforcement Investigations Center; and USGS method modified from EPA 1311), and simulated brackish water (USGS modification of EPA 1312 with simulated dilute sea water as the leaching fluid). These tests on the two Chalmette samples indicate that very low levels of metals would be leached from the samples when wet. Tests are under way to evaluate if metal release is greater from samples that have been dried completely, and from samples of the downtown New Orleans flood sediments.

\section{Preliminary organic analysis of the Chalmette samples indicates signatures that are largely typical of sediment from the Mississippi River delta}

Organic analyses to date (tables 7-9) indicate that the organic constituents of the Chalmette samples are dominated by biogenic (produced by living, non-human organisms) rather than anthropogenic (human-produced) or petrogenic (petroleum-derived) components. Some specific biogenic constituents are: suites of n-alkanes sourced from algae and land-based plant waxes, immature hopenes, the polycyclic aromatic hydrocarbon $(\mathrm{PAH})$ perylene, and plant derived sterols.

There are some markers for anthropogenic input, at much lower levels than the above, such as combustion PAHs, pervasive in the environment from the burning of fossil fuel. Also, some indications of sewage-related contamination are present (coprostanol, epicoprostanol), as are indications of slight contamination by oil-related compounds (low levels of oil-related hopanes and steranes). Some heavy (4-, 5-, 6-ring) PAHs having a elevated carcinogenic potential are present in the Chalmette samples but are relatively low and within the recommended sediment quality criteria proposed by the Washington Department of Ecology, except for benzo[a]pyrene, which although low, exceeds the standard. The PAH levels of the Chalmette samples are well below levels found by Mielke and others (2004) in pre-Katrina urban New Orleans soil samples.

Analyses for pesticides and pharmaceuticals are pending from other USGS laboratories for the Slidell, Violet, Chalmette, and downtown New Orleans samples.

\section{High iron sulfide contents indicate the Chalmette flood sediments may generate acid drainage when left in contact with rainfall and atmospheric oxygen}

USGS studies to date indicate that the Chalmette-area flood sediments contain quite elevated levels of pyrite (iron disulfide) and, prior to drying, elevated levels of acidvolatile sulfides (iron monosulfides) (Table 10). Sample Chalmette 1 contains as much as $4 \%$ by weight pyrite, and Chalmette 2 contains up to $1 \%$ by weight pyrite (table $3 a$ ). These abundances are common features of natural mud from marine marshes, and result from bacteria-driven reduction of seawater sulfate in the marsh mud, coupled with the 
reaction of the resulting aqueous sulfide with iron and other metals in the muds. This is consistent with the flood sediments having been transported into the Chalmette area from marshes to the east-northeast. Chalmette 1 contains little if any carbonate minerals, and Chalmette 2 only contains very small amounts of carbonate minerals. Both Chalmette samples also have abundant salt (sodium chloride) due to the evaporation of brackish water.

The abundance of pyrite and acid-volatile sulfide in the Chalmette samples indicates that, as the flood sediments continue to weather by reacting with rainwater and atmospheric oxygen, there is a strong potential for acidic pore waters and runoff waters to form. Such waters would likely have elevated concentrations of acid and sulfate (in the form of moderately concentrated sulfuric acid), along with iron, aluminum, and other metals leached from the sediment by the acid. These are common chemical processes that occur as pyrite-rich rocks weather to produce "acid-rock drainage" or ARD. The lack of carbonate minerals in the Chalmette 1 sediments also means that the sediments have very little capacity to self-neutralize the acid generated by sulfide oxidation.

Net acid production tests (table 11), similar to those originally developed to test whether sulfide-bearing mine waste piles would develop ARD, indicate that sample Chalmette 1 is very acid generating, and sample Chalmette 2 is slightly acid generating In contrast, the limited number of samples analyzed to date from the downtown New Orleans area do not appear to contain pyrite or acid-volatile sulfides, but do contain some calcium carbonate minerals.

These results indicate that the progressive chemical weathering of pyrite-rich Chalmette-area flood sediments, if either left in place or removed to an on-land storage facility exposed to rainfall and the atmosphere, may start to generate acidic solutions. The acid drainage could be somewhat corrosive to concrete and metals with which the sediments are in contact, and may be detrimental to vegetation and aquatic life affected by the drainage. In contrast to the Chalmette sediments, the downtown New Orleans sediments analyzed to date do not appear to pose a similar potential for generation of acid drainage. Further mineralogical characterization and acid-base accounting tests are underway on a broader number of samples from the greater New Orleans area to define the distribution of elevated iron sulfide levels in the sediment.

The potential for the Chalmette area samples to be acid-generating is most likely only a concern if the sediments are removed to an on-land disposal facility where they will be exposed to rainfall and the atmosphere. Sediments containing high levels of iron sulfides and low levels of carbonate minerals might best be considered as candidates for disposal into water-covered disposal areas or disposal facilities isolated from the atmosphere and rainfall.

\section{Other results are pending}

Results of additional chemical leach tests, microbial characterization studies, and organic contaminant analyses are currently pending.

\section{USGS Contacts}

Progress report: Geoff Plumlee, gplumlee@usgs.gov

SEM studies: Greg Meeker, gmeeker@usgs.gov 
XRD studies: George Breit, gbreit@usgs.gov; Steve Sutley, ssutley@usgs.gov

Inorganic chemical analyses: Paul Lamothe, plamothe@usgs.gov

Chemical leach tests: Phil Hageman, phageman@usgs.gov; Geoff Plumlee, gplumlee@usgs.gov

Organic chemical analyses: Ed Furlong, efurlong@usgs.gov, Bob Rosenbauer, brosenbauer@usgs.gov, Carl Orazio, corazio@usgs.gov

Additional contacts will be listed as other analytical results are completed.

\section{Other contributors}

Monique Adams, Murray Beasley, Mark Burkhardt, Zoe Ann Brown, Roger Clark, Jim Crock, Bob Eganhouse, Bill Foreman, Dale Griffin, Tammy Hannah, Todd Hoefen, JoAnn Holloway, Chris Ingersoll, Eric Livo, Elena Nielsen, Jim Ranville, Rick Sanzolone, Gregg Swayze, Joe Taggart, Dave Walters, Steve Werner, Tom Wildeman

\section{References Cited}

Chou, C-C., and Liu, Y-P., 2004, International Journal of Environmental and Analytical Chemistry, v. 84, p. 379-388.

Gessner, M.O., and Newell, S.Y., 2002, Biomass, growth rate, and production of filamentous fungi in plant litter, in Hurst, C.J., Crawford, R.L., Knudson, G., McInerney, M., Stetzenbach, L.D., eds., Manual of Environmental Microbiology, 2nd ed.: ASM Press, Washington, DC. P. 390-408.

Jeng, W.L., Huh, C.A., 2004, Lipids in suspended matter and sediments from the East China Sea Shelf: Organic Geochemistry, v. 35, p.647-660.

Jeng, W.L., Huh, C.A., 2001, Comparative study of sterols in shelf and slope sediments off northeastern Taiwan: Applied Geochemistry, v. 16, 95-108.

LDEQ, 2003, Risk Evaluation/Corrective Action Program (RECAP) Table 1, Screening option - Screening standards for soil and groundwater: http://www.deq.louisiana.gov/portal/Portals/0/technology/recap/2003/RECAP\%2020 03\%20Text\%20Table\%201.pdf

Lapakko, K.A., and Lawrence, R.W., 1993, Modification of the net-acid production (NAP) test, in Proceedings, British Columbia Mine Reclamation Symposium, Port Hardy, B.C., May 4-7, 1993, p. 145-159.

Lindsay, W.L., 1979, Chemical equilibria in soils: John Wiley and Sons, New York, 449 p.

Matsumoto, K., Yamada, K., and Ishiwatari. R., 2001, Sources of 24-methylcholest-5-en3b-ol in Japan Sea sediments over the past 30,000 years inferred from its carbon isotopic composition: Org. Geochem., v. 32, p. 259-269. 
Mielke, H.W., 1999, Lead in the inner cities: American Scientist, v. 87, p. 62-73.

Mielke, H.W., Wang, G., Gonzales, C.R., Powell, E.T., Le, B., and Quach, V.N., 2004, PAHs and metals in the soils of inner-city and suburban New Orleans, Louisiana, USA: Environmental Toxicology and Pharmacology, v. 18, p. 243-247.

Neff, J.M., 1979, Polycyclic aromatic hydrocarbons in the aquatic environment: sources, fates, and biological effects: Appl. Sci., London, UK, 262 p.

Netherlands, 1997, Circular on target values and intervention values for soil remediation, Annexes A-D. Netherlands Ministry of Housing, Spatial Planning and the Environment. http://international.vrom.nl/Docs/internationaal/annexS_I2000.pdf

Rugen, P.J., Stern, C.D., and Lamm, S.H., 1989, Comparative carcinogenicity of the PAHs as a basis for acceptable exposure levels (AELs) in drinking water: Regul Toxicol Pharmacol., v. 9, p. 273-283.

Shacklette, H.T., and Boerngen, J.G., 1984, Element concentrations in soils and other surficial materials of the conterminous United States: U.S. Geological Survey Professional Paper 1270, 105 pp.

USPHS, 1990, Toxicological profile for polycyclic aromatic hydrocarbons: U.S. Department of Health and Human Services, Public Health Service, Agency for Toxic Substances and Disease Registry, $231 \mathrm{p}$.

Washington State Dept. of Ecology, 2005, Sediment Quality Chemical Criteria: Toxics Cleanup Program, Sediment Management Unit, P.O. Box 47703, Olympia, WA 98504 


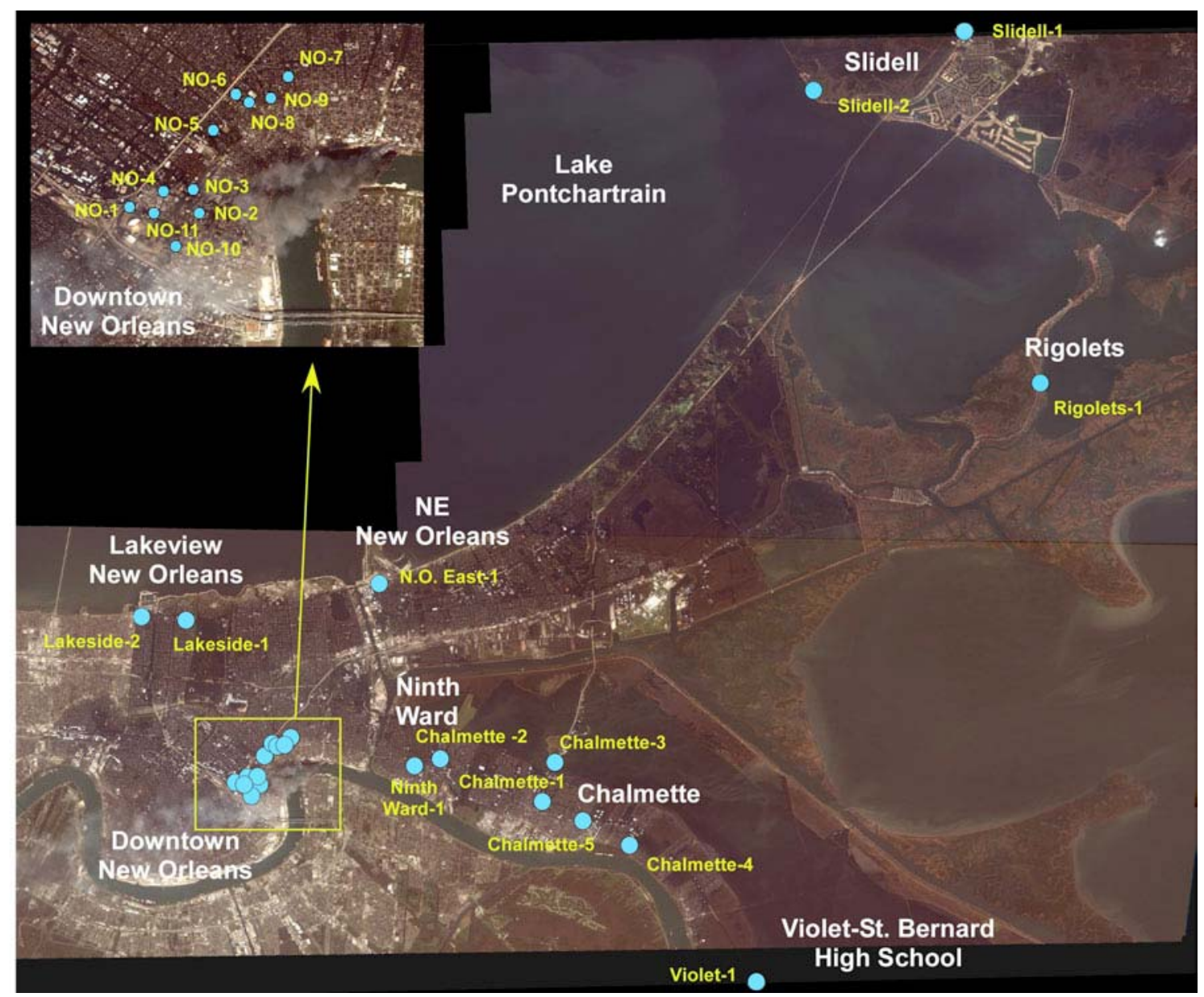

Figure 1. Locations of samples (blue circles) being analyzed to date in the USGS study, superimposed on a DigitalGlobe satellite image of the area from August 31, 2005. 


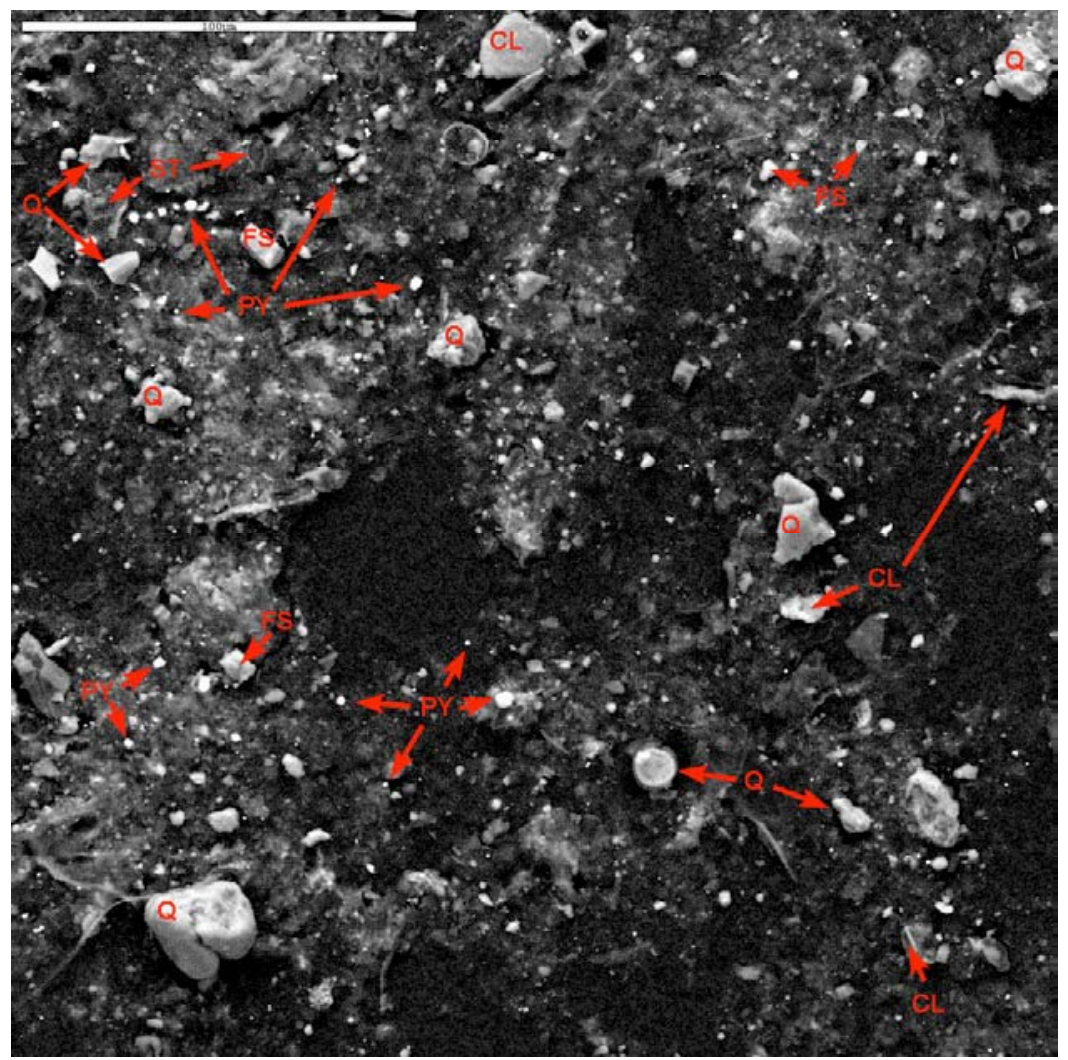

Figure 2. Backscattered electron micrograph of Chalmette composite sample 1. Image shows examples of mineral material (light gray) and unidentified organic material (darker gray). Typical mineral phases are labeled in the image. $\mathrm{Q}=$ quartz, $\mathrm{FS}=$ feldspar, $\mathrm{CL}=$ clay, $\mathrm{PY}=$ pyrite, $\mathrm{ST}=$ siliceous tests (skeletons) from microorganisms such as diatoms. Scale bar is 100 microns long.

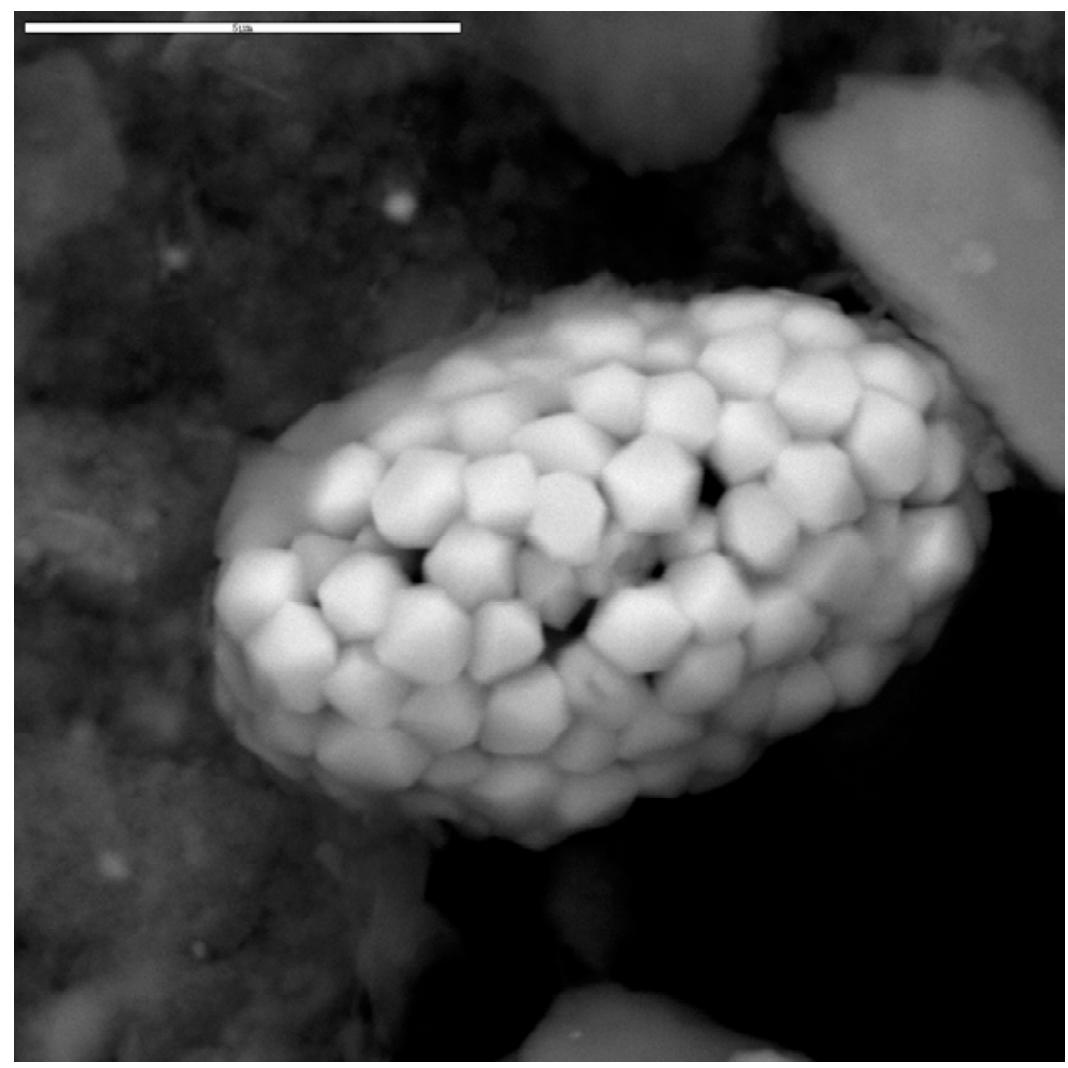

Figure 3. Backscattered electron micrograph showing an example of a pyrite framboid from sample Chalmette 1 with individual crystals $<1 \mathrm{~mm}$ in diameter (scale bar is 5 microns long). 


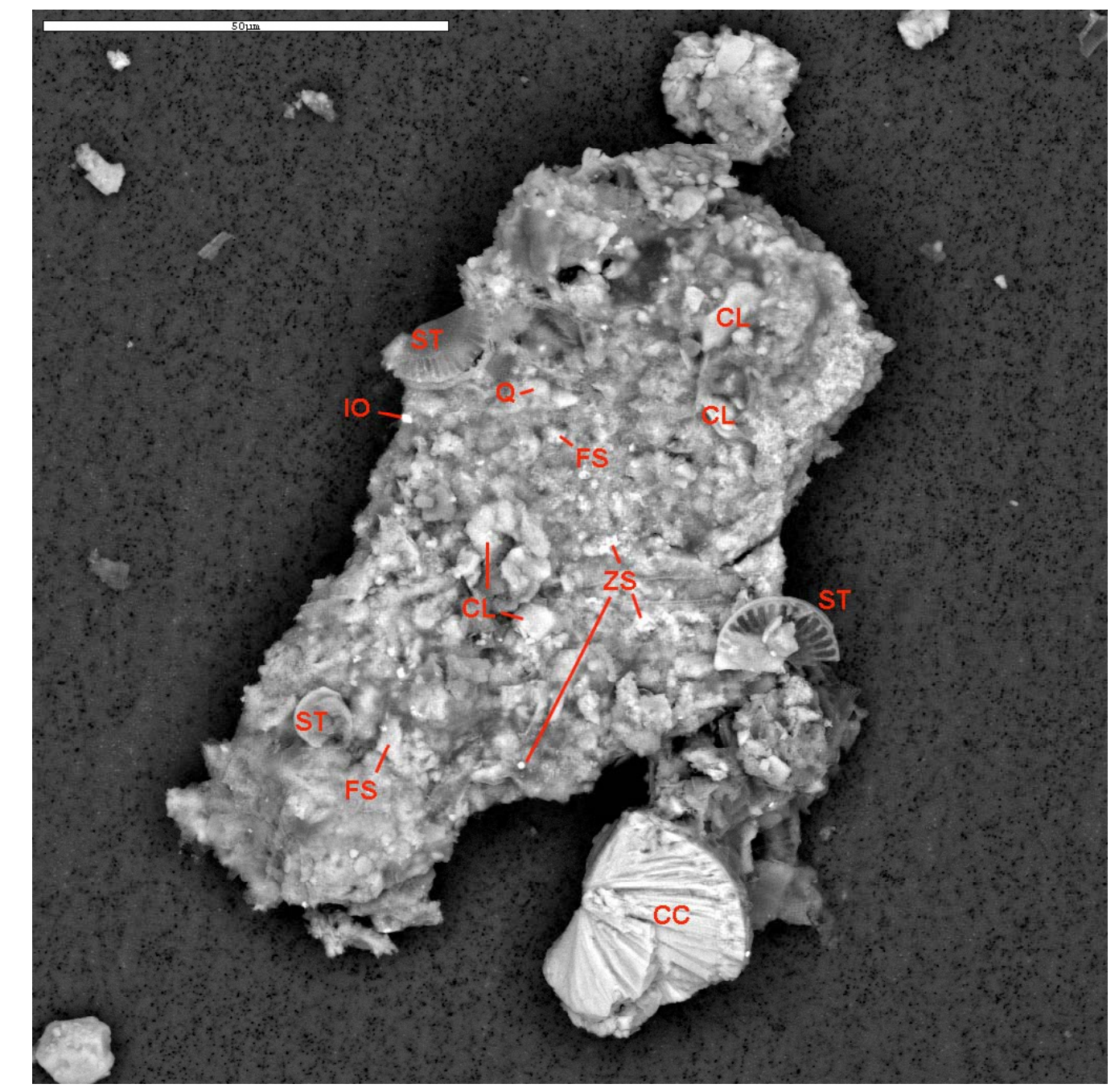

Figure 4. Typical aggregate grain of downtown New Orleans sub-sample NO7-B showing examples of primary components. $\mathrm{CL}=$ clay; $\mathrm{Q}=$ quartz; $\mathrm{FS}=$ feldspar; $\mathrm{CC}=$ calcium carbonate; $\mathrm{ST}=$ silica test; $\mathrm{ZS}=\mathrm{Zn}-\mathrm{S}$-rich; $\mathrm{IO}=$ iron oxide. Scale bar is 50 microns long. 


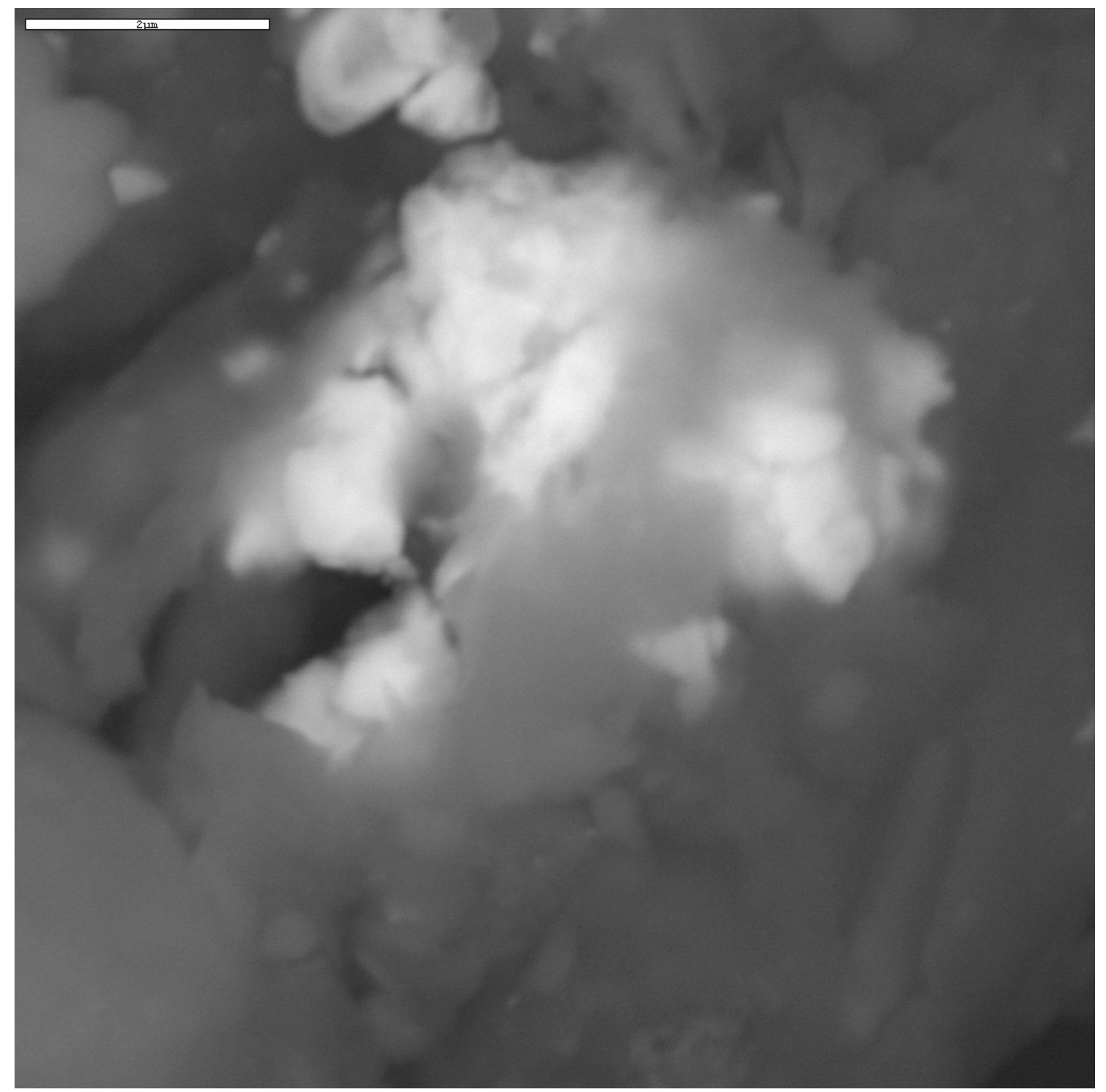

Figure 5. Backscattered electron image of lead-phosphorus-chlorine-rich particles (white) surrounded by clays and organic matter, from sample NO-7C. Scale bar is 2 microns long. 

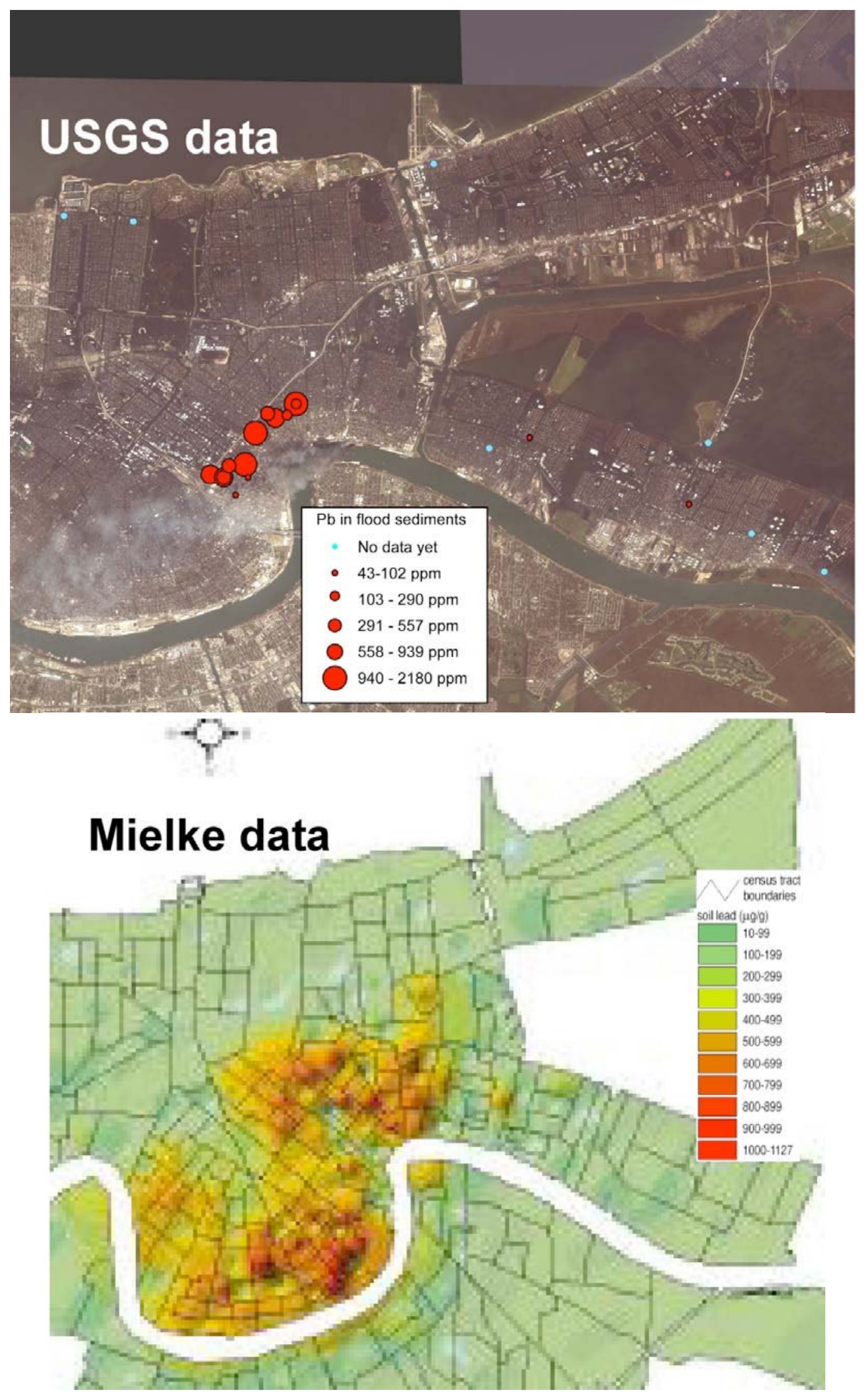

Figure 6. Map of lead concentrations measured by the USGS to date in New Orleans flood sediment samples (upper), compared to a gridded and digitally smoothed map of lead concentrations determined by Mielke and others (1999) for urban New Orleans soil samples collected in the 1990's. 

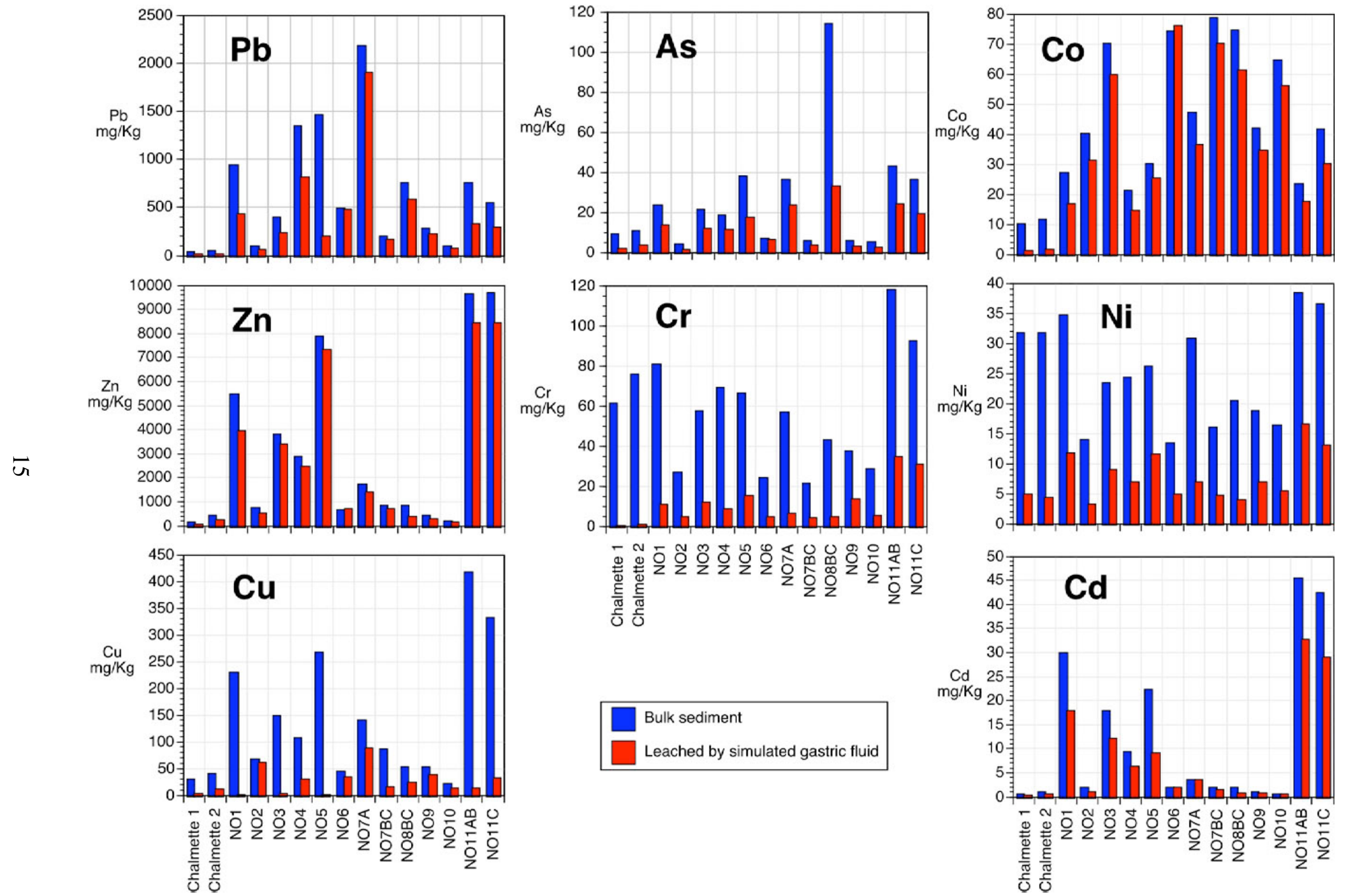

Figure 7. Plots of bulk metal concentrations (blue bars) compared to concentrations leached by simulated gastric fluids (red bars) in Katrina flood sediments. Note that scales of the concentration (y) axes vary between the plots for the different elements. 

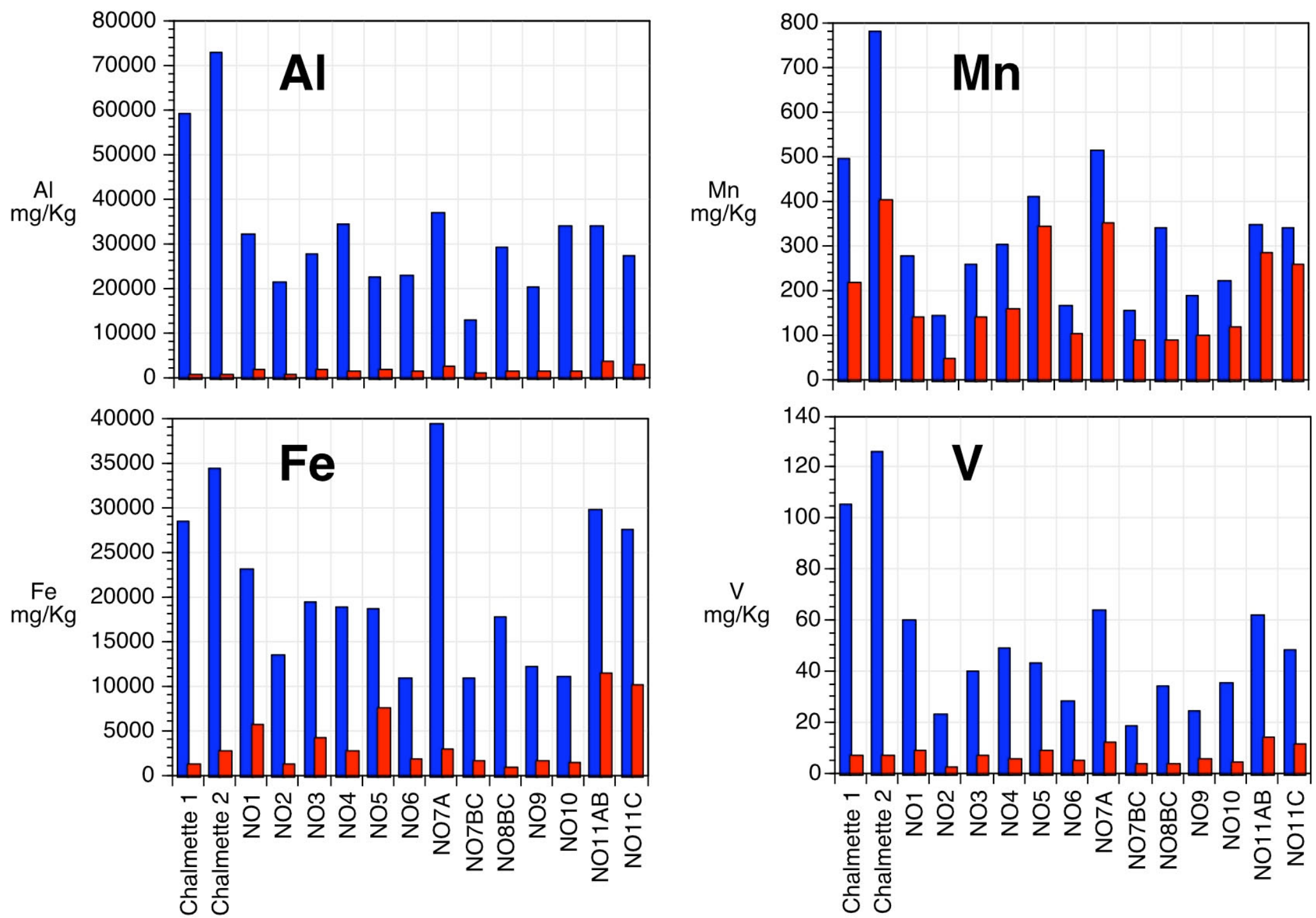

Bulk sediment

Leached by simulated gastric fluid

Figure 7, cont'd. Plots of bulk metal concentrations (blue bars) compared to concentrations leached by simulated gastric fluids (red bars) in Katrina flood sediments. Note that scales of the concentration (y) axes vary between the plots for the different elements. 


\section{Appendix A - Sediment sampling protocols.}

Contacts: John Lovelace, jlovelace@usgs.gov; Charlie Demas, cdemas@usgs.gov; Kathleen S. Smith (composite field sampling protocols), ksmith@usgs.gov

The flood sediments at eleven sites around downtown New Orleans (samples NO111, fig. 1, table 1) were sampled on September 15, 2005, by John Lovelace and Mike Ross (USGS Louisiana Water Science Center). At each locality, subsamples were collected from 3 separate locations up to 30 meters apart, with the location of the central point for each locality determined using a GPS unit. Each subsample was collected using a Teflon ${ }^{\circledR}$ scoop and placed into a new pint-sized polyethylene food-grade container. At most localities sampled, there was a mix of dry and wet subsamples. The samples from each locality were stored on ice in a cooler and then later transferred to a refrigerator for storage at $4^{\circ} \mathrm{C}$ until shipment on ice to USGS labs in Denver.

Flood sediment samples were collected from two localities in the Chalmette area (Chalmette 1 and 2, fig. 1) on September 16, 2005. Forty subsamples were collected at each locality using Teflon ${ }^{\circledR}$ scoops, and each subsample was placed into a new pint-size polyethylene food-grade container. The subsamples were typically collected at 1-meter intervals along each of 4 transects extending radially from a street intersection. A GPS was used to determine the latitude and longitude of the center point and the end points of each transect. This sampling approach was originally developed for the sampling of highly variable mine-waste piles, so as to provide a statistically meaningful representation of a much larger area with great internal variability. The flood sediments at Chalmette 1 were still predominantly wet with only the top surface being dry. The flood sediments at Chalmette 2 were also moist to wet, but with a somewhat greater proportion of dry material than at Chalmette 1 . The samples from each locality were stored on ice in a cooler and then later transferred to a refrigerator for storage at $4^{\circ} \mathrm{C}$ until shipment on ice to USGS labs in Denver.

Flood sediment samples were collected from the Slidell, Rigolets, northern and northeastern New Orleans, Ninth Ward, Chalmette, and St. Bernard High School areas on October 6 and 7, 2005. Forty subsamples were collected at each locality, at spatially regular intervals along each of 4 transects. In contrast to the previous two sampling efforts, these samples were composited as they were collected. One composite was collected from each locality using a Teflon scoop into a 4-liter polyethylene wide mouth jar. Another composite was collected at each locality using a clean stainless steel spoon into a 1-liter brown glass jar. The samples from each locality were stored on ice in a cooler and then later transferred to a refrigerator for storage at $4^{\circ} \mathrm{C}$ until shipment on ice to USGS labs in Denver. 
Table 1. Locations of samples analyzed in the USGS Katrina flood sediment characterization study. Contact for sampling: John Lovelace (jlovelace@usgs.gov).

\begin{tabular}{|c|c|c|c|c|}
\hline sample & Date collected & Lat & Long & Site description \\
\hline Chalmette 1 & $9 / 16 / 05$ & 29.94563889 & 89.95975 & Intersection of St. Jean Baptiste and Tourneforte St., Chalmette \\
\hline Chalmette 1 & $10 / 6 / 05$ & 29.94563889 & 89.95975 & Intersection of St. Jean Baptiste and Tourneforte St., Chalmette \\
\hline Chalmette 2 & $9 / 16 / 05$ & 29.96266667 & 90.00047222 & 1600 Block of Ayock St., Arabi, LA \\
\hline Chalmette 3 & $10 / 6 / 05$ & 29.96138889 & 89.95472222 & Paris Road \\
\hline Chalmette 4 & $10 / 6 / 05$ & 29.92833333 & 89.925 & NP Trist Middle School \\
\hline Chalmette 5 & $10 / 7 / 05$ & 29.93805556 & 89.94361111 & Ventura Drive and Judge Perez \\
\hline NO1 & $9 / 15 / 05$ & 29.9532778 & 90.082278 & New Orleans, Poydras Ave. at Superdome \\
\hline $\mathrm{NO} 2$ & $9 / 15 / 05$ & 29.95247222 & 90.07263889 & New Orleans, Barrone \& Gravier Sts. \\
\hline NO3 & $9 / 15 / 05$ & 29.9555 & 90.0775 & New Orleans, Tulane \& Lasalle @ Tulane Med Center \\
\hline NO4 & $9 / 15 / 05$ & 29.95577778 & 90.07338889 & New Orleans, Canal \& Basin Sts. \\
\hline NO5 & $9 / 15 / 05$ & 29.96388889 & 90.07069444 & New Orleans, N. Villere \& St. Anne Sts. \\
\hline NO6 & $9 / 15 / 05$ & 29.96886111 & 90.06758333 & New Orleans, Esplanade \& N. Robertson \\
\hline NO7 & $9 / 15 / 05$ & 29.97130556 & 90.06030556 & New Orleans, Pauger \& N. Villere \\
\hline NO8 & $9 / 15 / 05$ & 29.96772222 & 90.06569444 & New Orleans, Esplanade \& Marais \\
\hline NO9 & $9 / 15 / 05$ & 29.96838889 & 90.06266667 & New Orleans, Annette, St. Claude, \& St. Bernard \\
\hline NO10 & $9 / 15 / 05$ & 29.94794444 & 90.07586111 & New Orleans, Rampart \& Girod \\
\hline NO11 & $9 / 15 / 05$ & 29.95252778 & 90.07886111 & New Orleans, Freret \& Perdido \\
\hline Lakeside 1 & $10 / 7 / 05$ & 30.01944444 & 90.11972222 & Kenison \& North Hammond Highway \\
\hline Lakeside 2 & $10 / 7 / 05$ & 30.01805556 & 90.10194444 & Robert E Lee Blvd and Marshall Foch \\
\hline N.O. East 1 & $10 / 7 / 05$ & 30.03277778 & 90.025 & 8006 W. Laverne \\
\hline Violet 1 & $10 / 7 / 05$ & 29.87388889 & 89.87444444 & St. Bernard High School \\
\hline Ninth Ward 1 & $10 / 7 / 05$ & 29.96 & 90.01083333 & Tupelo and St Claude Ave. \\
\hline Slidell1 & $10 / 6 / 05$ & 30.25305556 & 89.79138889 & 3805 Highway 11 \\
\hline Slidell2 & $10 / 6 / 05$ & 30.22944444 & 89.85166667 & Carr Drive \\
\hline Rigolets1 & $10 / 6 / 05$ & 30.11277778 & 89.76111111 & Highway 90 \\
\hline
\end{tabular}


Table 2. Types of analyses used to characterize Katrina flood sediments. GD - USGS Geologic Discipline, BRD - USGS Biological Reesources Discipline; WRD - USGS Water Resources Discipline

\begin{tabular}{|c|c|c|}
\hline Analysis type & Lab & Contact \\
\hline $\begin{array}{l}\text { Inorganic chemistry, } 42 \text { element, 4-acid } \\
\text { digestion, ICP-MS }\end{array}$ & Denver GD & $\begin{array}{l}\text { Paul Lamothe, } \\
\text { plamothe@usgs.gov }\end{array}$ \\
\hline XRF major, trace element chemistry & Denver GD & $\begin{array}{l}\text { Joe Taggart, } \\
\text { jtaggart@usgs.gov }\end{array}$ \\
\hline Total Hg, Methyl Hg & Denver GD & Jim Crock, jcrock@usgs.gov \\
\hline Grain size analysis & Menlo GD & $\begin{array}{l}\text { Mike Torresan, } \\
\text { mtorresan@usgs.gov }\end{array}$ \\
\hline X-ray diffraction mineralogy & Denver GD & $\begin{array}{l}\text { Steve Sutley, } \\
\text { ssutley@usgs.gov }\end{array}$ \\
\hline $\begin{array}{l}\text { Scanning electron microscope } \\
\text { mineralogy, quantitative electron probe } \\
\text { microanalysis }\end{array}$ & Denver GD & $\begin{array}{l}\text { Greg Meeker, } \\
\text { gmeeker@usgs.gov }\end{array}$ \\
\hline $\begin{array}{l}\text { Gamma spectrometry screening for } \\
\text { radionuclides ( } U, \mathrm{Ra}, \mathrm{Pb} 210 \text {, etc.) }\end{array}$ & Denver GD & $\begin{array}{l}\text { Jim Budahn, } \\
\text { jbudahn@usgs.gov }\end{array}$ \\
\hline $\begin{array}{l}\text { Field-flow fractionation -Element } \\
\text { distributions over fine-sil/colloid size } \\
\text { continuum for wet and dry sediments }\end{array}$ & Denver GD & Lopaka Lee, rclee@usgs.gov \\
\hline Reflectance spectroscopy & Denver GD & $\begin{array}{l}\text { Roger Clark, } \\
\text { rclark@usgs.gov. Gregg } \\
\text { Swayze, gswayze@usgs.gov. }\end{array}$ \\
\hline 4-step sequential extraction $* *$ & Denver GD & $\begin{array}{l}\text { Rick Sanzolone, } \\
\text { rsanzolo@usgs.gov }\end{array}$ \\
\hline Forms of sulfur & Denver GD & $\begin{array}{l}\text { Michelle Tuttle, } \\
\text { mtuttle@usgs.gov }\end{array}$ \\
\hline Carbonate and organic carbon & Denver GD & $\begin{array}{l}\text { Zoe Ann Brown, } \\
\text { zbrown@usgs.gov }\end{array}$ \\
\hline EPA SPLP (1312) metals leach** & Denver GD & $\begin{array}{l}\text { Phil Hageman, } \\
\text { phageman@usgs.gov }\end{array}$ \\
\hline USGS Field leach test for metals** & Denver GD & $\begin{array}{l}\text { Phil Hageman, } \\
\text { phageman@usgs.gov }\end{array}$ \\
\hline Synthetic 6 ppt sea water leach** & Denver GD & $\begin{array}{l}\text { Phil Hageman, } \\
\text { phageman@usgs.gov }\end{array}$ \\
\hline Field TCLP/EPA TCLP verification** & Colo. School of Mines; EPA NEIC & $\begin{array}{l}\text { Jim Ranville, } \\
\text { jranvill@mines.edu, Tom } \\
\text { Wildeman, } \\
\text { twildema@mines.edu }\end{array}$ \\
\hline $\begin{array}{l}\text { ELISA screening for carcinogenic PAH's, } \\
\text { DDT/DDE, and PCB }\end{array}$ & Denver GD & $\begin{array}{l}\text { LaDonna Choate, } \\
\text { Ichoate@usgs.gov }\end{array}$ \\
\hline Simulated gastric fluid leach** & Denver GD & $\begin{array}{l}\text { Geoff Plumlee, } \\
\text { gplumlee@usgs.gov }\end{array}$ \\
\hline Simulated lung fluid leach** & Denver GD & $\begin{array}{l}\text { Geoff Plumlee, } \\
\text { gplumlee@usgs.gov }\end{array}$ \\
\hline Forms of cyanide using alkaline leach & Denver GD & $\begin{array}{l}\text { Craig J ohnson, } \\
\text { cjohnso@usgs.gov }\end{array}$ \\
\hline $\begin{array}{l}\text { PCR DNA molecular microbial screening } \\
\text { for specific pathogens, common } \\
\text { microbes }\end{array}$ & St. Petersburg GD & $\begin{array}{l}\text { Dale Griffin, } \\
\text { dgriffin@usgs.gov }\end{array}$ \\
\hline
\end{tabular}


Table 2. Types of analyses used to characterize Katrina flood sediments. GD - USGS Geologic Discipline, BRD - USGS Biological Reesources Discipline; WRD - USGS Water Resources Discipline

\begin{tabular}{|c|c|c|}
\hline Analysis type & Lab & Contact \\
\hline $\begin{array}{l}\text { PLFA microbial analysis for microbial } \\
\text { communities }\end{array}$ & GD Denver, UC Davis & $\begin{array}{l}\text { JoAnn Holloway, } \\
\text { jholloway@usgs.gov }\end{array}$ \\
\hline $\begin{array}{l}\text { L28-d whole-sediment toxicity tests, } \\
\text { amphipood Hyalella azteca }\end{array}$ & Columbia BRD & $\begin{array}{l}\text { Chris Ingersoll, } \\
\text { cingersoll@usgs.gov }\end{array}$ \\
\hline $\begin{array}{l}\text { PAH's, polychlorinated biphenyls } \\
\text { (congener-specific, 80+congeners), } \\
\text { volatile aromatic hydrocarbons, natural } \\
\text { biomarkers (hydrocarbons, fatty } \\
\text { alcohols, hydroxy acids, sterls), } \\
\text { molecular markers of municipal waste } \\
\text { (fecal sterols, long-chain alkylbenzenes, } \\
\text { vitamin E acetate), CHN analysis, } \\
\text { qualitative analysis of complex organic } \\
\text { chemical mixtures (GCxGC/ToFMS) }\end{array}$ & WRD Reston & $\begin{array}{l}\text { Bob Eganhouse, } \\
\text { eganhous@usgs.gov }\end{array}$ \\
\hline $\begin{array}{l}\text { Crude oil, petroleum products, PAH's, } \\
\text { sterols (eg coprostanol), current use and } \\
\text { legacy ag / urban contaminants } \\
\text { (organophosphates, carbamates, } \\
\text { triazines, chloracetanilides, DDT) }\end{array}$ & GD Menlo Park & $\begin{array}{l}\text { Bob Rosenbauer, } \\
\text { brosenbauer@usgs.gov }\end{array}$ \\
\hline $\begin{array}{l}\text { Dioxins/furans, PBDE's, PCB's and } \\
\text { chlorinated pesticides, semivolatile } \\
\text { organic "unknowns" }\end{array}$ & BRD Columbia & $\begin{array}{l}\text { Cari Orazio, } \\
\text { corazio@usgs.gov }\end{array}$ \\
\hline $\begin{array}{l}\text { WRD Schedule } 2501-O C \text { (insecticides), } \\
\text { WRD Schedule } 2502 \text { - Semivolatiles, } \\
\text { WRD LC8045 PCB Congeners, } \\
\text { wastewater compounds (schedule } \\
\text { 1433), PAH and Alkyl PAH, musks and } \\
\text { fragrances, human health } \\
\text { pharmaceuticals, antibiotics. As } \\
\text { appropriate, some organic analyses will } \\
\text { be run on leachates. }\end{array}$ & $\begin{array}{l}\text { WRD National Water Quality } \\
\text { Laboratory (NWQL) }\end{array}$ & $\begin{array}{l}\text { Ed Furlonq, } \\
\text { efurlong@usgs.gov }\end{array}$ \\
\hline Organics on TCLP leach & WRD NWQL & $\begin{array}{l}\text { Ed Furlong, } \\
\text { efurlong@usgs.gov }\end{array}$ \\
\hline $\begin{array}{l}\text { Reactive oxygen species generation, cell } \\
\text { line toxicity testing }\end{array}$ & $\begin{array}{c}\text { State University of New York Stony } \\
\text { Brook }\end{array}$ & $\begin{array}{l}\text { Martin Schoonen, } \\
\text { schoonen@stonybrook.edu }\end{array}$ \\
\hline
\end{tabular}


Table 3a. Analytical results for quantitative X-Ray Diffraction (XRD) . Analyst - George Breit

(gbreit@usgs.gov).nd -Not detected. Analyses performed on Siemens D500 Kristalloflex, using Ni

filtered, Cu Ka radiation scanning from 4 to 64 degrees $2 q, 0.02$ degrees per step and 3 second count time pre step.

\begin{tabular}{|l|c|c|c|c|}
\hline Comment & $\begin{array}{c}\text { Chalmette 1 } \\
\text { sample freeze-dried } \\
\text { from wet }\end{array}$ & $\begin{array}{c}\text { Chalmette 2 } \\
\text { sample freeze- } \\
\text { dried from wet }\end{array}$ & $\begin{array}{c}\text { Nomple freeze-dried } \\
\text { from wet }\end{array}$ & $\begin{array}{c}\text { sample dry when } \\
\text { collected }\end{array}$ \\
\hline Quartz & wt. \% & wt. \% & wt. \% & wt. \% \\
\hline Pyrite & 16 & 24 & 80 & 16 \\
\hline Halite & 4 & 1 & ND & ND \\
\hline Hematite & 2 & 1 & $<0.5$ & 2 \\
\hline Gypsum & ND & 0.4 & 2 & ND \\
\hline Ferroan(?) dolomite & ND & ND & ND & ND \\
\hline Potassium feldspar & ND & ND & detected & ND? \\
\hline $\begin{array}{l}\text { Plagioclase feldspar } \\
\text { Illite + chlorite }\end{array}$ & detected & detected & detected & detected \\
\hline $\begin{array}{l}\text { Non-crystalline } \\
\text { material (silica } \\
\text { remnants and organic } \\
\text { matter }\end{array}$ & detected & detected & detected & \\
\hline
\end{tabular}




\begin{tabular}{|c|c|c|c|c|c|c|c|c|c|c|c|c|c|c|c|}
\hline & $\begin{array}{c}\text { Chalmette } \\
1 \\
\end{array}$ & $\begin{array}{c}\text { Chalmette } \\
\mathbf{2} \\
\end{array}$ & NO-1 & NO-2 & NO-3 & NO-4 & NO-5 & NO-6 & NO-7A & NO-7BC & NO-8 BC & NO-9 & NO-10 & NO-11 AB & N011c \\
\hline Quartz & Major & Major & Major & Major & Major & Major & Major & Major & Major & Major & Major & Major & Major & Major & Major \\
\hline Calcite & ND & ND & Minor & Minor & Trace & Trace & Minor & Trace & Trace & Minor & Trace & Minor & Trace & Minor & Minor \\
\hline Albite & Trace & Trace & Trace & Trace & Trace & Trace & Trace & Trace & Trace & Trace & Trace & Trace & Trace & Trace & Trace \\
\hline Ankerite & ND & ND & ND & ND & ND & ND & Trace & ND & ND & ND & $?$ & $?$ & ND & ND & ND \\
\hline Dolomite & ND & ND & ND & ND & ND & ND & $?$ & ND & ND & ND & Trace & Trace & ND & ND & ND \\
\hline Orthoclase & Trace & Trace & Trace & Trace & Trace & Trace & ND & Trace & Trace & Trace & Trace & Trace & Trace & Trace & Trace \\
\hline Muscovite & Trace & Trace & Trace & Trace & Trace & Trace & Trace & Trace & Trace & Trace & Trace & Trace & Trace & Trace & Trace \\
\hline Kaolinite & Trace & Trace & $?$ & $?$ & ND & $?$ & $?$ & $?$ & ND & $?$ & $?$ & ND & $?$ & $?$ & $?$ \\
\hline Halite & Trace & Trace & $?$ & ND & ND & ND & Trace & ND & Trace & Trace & ND & ND & ND & $?$ & Minor \\
\hline Pyrite & Trace & Trace & ND & ND & ND & ND & ND & ND & ND & $\mathrm{ND}$ & ND & ND & ND & ND & ND \\
\hline Montmorillonite & Minor & Minor & ND & ND & ND & ND & ND & ND & ND & ND & ND & ND & ND & ND & ND \\
\hline Hematite & ND & ND & ND & ND & ND & ND & ND & ND & $?$ & $?$ & ND & ND & $?$ & ND & ND \\
\hline Gypsum & ND & ND & ND & ND & ND & ND & $?$ & ND & ND & ND & ND & ND & ND & ND & ND \\
\hline
\end{tabular}




\begin{tabular}{|c|c|c|c|c|}
\hline & Comment & Size distribution & Mineralogy & $\begin{array}{l}\text { Other microscopic materials, including plant } \\
\text { matter and commercial or man-made } \\
\text { materials }\end{array}$ \\
\hline Chalmette 1 & $\begin{array}{l}\text { sample freeze } \\
\text { dried from wet }\end{array}$ & $\begin{array}{l}\text { All particles less } \\
\text { than } 20 \text { microns; } \\
\text { most are less than } 5 \\
10 \text { microns }\end{array}$ & $\begin{array}{l}\text { Quartz; clays (including illites, smectites, } \\
\text { possible chlorites); ailica with varying amounts } \\
\text { of aluminum (may be biogenic or with clay); } \\
\text { ailica diatom tests; feldspars, including } \\
\text { potassium- and sodium-rich; relatively abundant } \\
\text { pyrite (mostly framboidal); }\end{array}$ & Pollen grains (rare); glass fibers (rare) \\
\hline Chalmette 2 & $\begin{array}{l}\text { sample freeze } \\
\text { dried from wet }\end{array}$ & $\begin{array}{l}\text { All particles less } \\
\text { than } \sim 20 \text { microns; } \\
\text { most are less than } 5 \\
10 \text { microns }\end{array}$ & $\begin{array}{l}\text { Quartz; clays (including illites, smectites, } \\
\text { possible chlorites); silica with varying amounts } \\
\text { of aluminum (may be biogenic or with clay); } \\
\text { silica diatom tests; feldspars, including } \\
\text { potassium- and sodium-rich; minor pyrite (few } \\
\text { framboids, more micron-sized single crystals } \\
\text { which could be disaggregated from framboids) }\end{array}$ & \\
\hline NO-5 & $\begin{array}{l}\text { Sample air } \\
\text { dried }\end{array}$ & $\begin{array}{l}\text { Most particles }<5 \\
\text { microns unless an } \\
\text { aggregate grain }\end{array}$ & $\begin{array}{l}\text { Quartz; clays (kaolinites, illites, smectites); } \\
\text { apatite (calcium phosphate); silica with varying } \\
\text { amounts of aluminum (may be biogenic or with } \\
\text { kaolinite clay); silica diatom tests; feldspars, } \\
\text { including potassium- and sodium-rich; calcium } \\
\text { carbonates; silica, aluminum, magnesium, iron } \\
\text { compounds. Heavy metal compounds include: } \\
\text { metallic lead; Zn-S-C particles (zinc sulfate- } \\
\text { carbonate?); barite; iron oxides; and } \\
\text { phosphates. Many of the heavy metals occur in } \\
\text { particles less than }<0.5 \text { microns in size. }\end{array}$ & some slag wool, silica diatoms, \\
\hline
\end{tabular}




\begin{tabular}{|c|c|c|c|c|}
\hline & Comment & Size distribution & Mineralogy & $\begin{array}{l}\text { Other microscopic materials, including plant } \\
\text { matter and commercial or man-made } \\
\text { materials }\end{array}$ \\
\hline NO-7A & $\begin{array}{l}\text { sample freeze- } \\
\text { dried from wet }\end{array}$ & $\begin{array}{l}\text { Most particles }<5 \\
\text { microns unless an } \\
\text { agregrate grain }\end{array}$ & $\begin{array}{l}\text { Quartz; clays (kaolinites, illites, smectites); } \\
\text { apatite (calcium phosphate); silica with varying } \\
\text { amounts of aluminum (may be biogenic or with } \\
\text { kaolinite clay); silica diatom tests; feldspars, } \\
\text { including potassium- and sodium-rich; calcium } \\
\text { and magnesium carbonates; silica, alumium, } \\
\text { magnesium, iron compound. Heavy metal } \\
\text { compounds include: Zn-S-C particles (zinc } \\
\text { sulfate-carbonate?); barite; iron oxides; lead } \\
\text { generally with calcium and phosphorus; copper } \\
\text { sulfates; apatite; titanium oxide; and zircon. }\end{array}$ & Carbon fragments (plant material?) \\
\hline NO-7C & & & $\begin{array}{l}\text { Quartz; clays; titanium oxides; lead-phosphate- } \\
\text { chloride particles; lead or lead oxide particles. }\end{array}$ & \\
\hline NO-11 comp & & & $\begin{array}{l}\text { Quartz; clays (kaolinites, illites, smectites); } \\
\text { apatite (calcium phosphate); silica with varying } \\
\text { amounts of aluminum (may be biogenic or with } \\
\text { kaolinite clay); feldspars, including potassium- } \\
\text { and sodium-rich; calcium and magnesium } \\
\text { carbonates. Heavy metal compounds include: } \\
\text { barite; iron oxides (some of which are relatively } \\
\text { large, } 40 \mu \mathrm{m} \text { ); iron and titanium oxides; } \\
\text { metallic lead; one particle of tin. }\end{array}$ & Carbon fragments (plant material?) \\
\hline
\end{tabular}




\begin{tabular}{|c|c|c|c|c|}
\hline Table 4. & $\begin{array}{l}\text { Inorganic analytical results for USGS samples, compared to nearby EPA samples, mea } \\
\text { and non-industrial LDEQ (2003) Soil Screening Standards. WDXRF-wavelength disper } \\
\text { coupled plasma-mass spectrometry; CVAA - cold vapor atomic absorption spectrosco }\end{array}$ & $\begin{array}{l}\text { JS soils, Nethe } \\
\text { ex-ray fluoresce } \\
\text { NA - Not analyz }\end{array}$ & $\begin{array}{l}\text { Ids (1997) soil c } \\
\text { e; ICP-MS - ind } \\
\text { NR - Not repor }\end{array}$ & $\begin{array}{l}\text { lity criteria, } \\
\text { ively }\end{array}$ \\
\hline Field No. & Sample Description & $\begin{array}{c}\text { SiO2 } \\
\text { wt. \% } \\
\text { WDXRF }\end{array}$ & $\begin{array}{l}\text { Al2O3 } \\
\text { wt. \% } \\
\text { WDXRF }\end{array}$ & $\begin{array}{c}\text { Fe2O3 } \\
\text { wt. \% } \\
\text { WDXRF }\end{array}$ \\
\hline Chalmette 1 & Katrina flood sediment, St. Jean Baptiste and Buffon Sts., Chalmette, LA & 43.2 & 11.1 & 4.1 \\
\hline Chalmette 1d & Sample duplicate & 43.4 & 11.1 & 4.1 \\
\hline EPA 8939* & EPA Katrina flood sediment, W Josephine St. & $\mathrm{NR}$ & NR & $\mathrm{NR}$ \\
\hline Chalmette 2 & Katrina flood sediment, Ayock St., Arabi, LA & 54.1 & 13.3 & 4.82 \\
\hline Chalmette 2d & Sample duplicate & 54.0 & 13.2 & 4.79 \\
\hline EPA $10174^{* *}$ & EPA Katrina flood sediment, 1921 Angela St. & NR & NR & NR \\
\hline $\mathrm{NO1}$ & New Orleans, Poydras Ave. at Superdome & 42.6 & 5.39 & 2.87 \\
\hline EPA 8490 & EPA 8490 Superdome & $\mathrm{NR}$ & NR & NR \\
\hline $\mathrm{NO2}$ & New Orleans, Barrone \& Gravier Sts. & 79.7 & 3.74 & 1.77 \\
\hline NO3 & New Orleans, Tulane \& LaSalle @ Tulane Med Center & 59.9 & 4.69 & 2.47 \\
\hline $\mathrm{NO} 4$ & New Orleans, Canal \& Basin Sts. & 63.6 & 5.80 & 2.38 \\
\hline NO5 & New Orleans, N. Villere \& St. Anne Sts. & 43.3 & 3.96 & 2.39 \\
\hline EPA 8493 & EPA Treme \& St. Philip, 9-11-05 & $\mathrm{NR}$ & $N R$ & $N R$ \\
\hline NO6 & New Orleans, Espalanade \& N. Robertson & 79.6 & 4.29 & 1.50 \\
\hline EPA 8910 & EPA Gov. Nichols \& Clairborne, 9-12-05 & $\mathrm{NR}$ & NR & NR \\
\hline NO7A & New Orleans, Pauger \& N. Villere & 57.4 & 6.61 & 5.11 \\
\hline NO7BC & New Orleans, Pauger \& N. Villere & 75.3 & 2.25 & 1.47 \\
\hline EPA 8912 & Touro \& Clairborne, 9-12-05 & $N R$ & $N R$ & $N R$ \\
\hline $\mathrm{NO8BC}$ & New Orleans, Esplanade \& Marais & 70.0 & 5.10 & 2.35 \\
\hline NO9 & New Orleans, Annette, St. Claude, \& St. Bernard & 71.8 & 3.38 & 1.55 \\
\hline EPA 8911 & EPA 8911, St. Anthony \& St. Claude & NR & NR & NR \\
\hline NO10 & New Orleans, Rampart \& Girod & 75.8 & 5.91 & 1.48 \\
\hline $\mathrm{NO11AB}$ & New Orleans, Freret \& Perdido & 35.4 & 6.22 & 4.00 \\
\hline $\mathrm{NO11C}$ & New Orleans, Freret \& Perdido & 40.8 & 4.70 & 3.49 \\
\hline US Mean soil & Shacklette \& Boerngen (1984); BOLD values from Lindsay (1979) & NR & NR & NR \\
\hline Netherlands & Tentative soil quality criteria, limiting value for soil quality & NR & NR & NR \\
\hline LDEQ & Louisiana Dept. Env. Qual. Soil Screening Standard & $N R$ & $\mathrm{NR}$ & $\mathrm{NR}$ \\
\hline
\end{tabular}




\begin{tabular}{|c|c|c|c|c|c|c|c|}
\hline Field No. & $\begin{array}{c}\text { MgO } \\
\text { wt. \% } \\
\text { WDXRF }\end{array}$ & $\begin{array}{c}\text { CaO } \\
\text { wt. \% } \\
\text { WDXRF }\end{array}$ & $\begin{array}{c}\text { Na20 } \\
\text { wt. \% } \\
\text { WDXRF }\end{array}$ & $\begin{array}{c}\text { K20 } \\
\text { wt. \% } \\
\text { WDXRF }\end{array}$ & $\begin{array}{c}\text { TiO2 } \\
\text { wt. \% } \\
\text { WDXRF }\end{array}$ & $\begin{array}{c}\text { P205 } \\
\text { wt. \% } \\
\text { WDXRF }\end{array}$ & $\begin{array}{c}\text { MnO } \\
\text { wt. \% } \\
\text { WDXRF }\end{array}$ \\
\hline Chalmette 1 & 1.97 & 0.91 & 2.69 & 1.80 & 0.48 & 0.23 & 0.06 \\
\hline Chalmette 1d & 1.99 & 0.92 & 2.66 & 1.80 & 0.48 & 0.22 & 0.06 \\
\hline EPA 8939* & NR & $\mathrm{NR}$ & $\mathrm{NR}$ & $\mathrm{NR}$ & NR & NR & NR \\
\hline Chalmette 2 & 2.01 & 1.29 & 1.87 & 2.27 & 0.58 & 0.20 & 0.10 \\
\hline Chalmette 2d & 2.02 & 1.28 & 1.90 & 2.26 & 0.58 & 0.21 & 0.10 \\
\hline EPA $10174^{* *}$ & $N R$ & $\mathrm{NR}$ & $\mathrm{NR}$ & $N R$ & $N R$ & $N R$ & NR \\
\hline $\mathrm{NO} 1$ & 1.23 & 4.45 & 1.59 & 0.92 & 0.34 & 0.39 & 0.03 \\
\hline EPA 8490 & $\mathrm{NR}$ & $\mathrm{NR}$ & $\mathrm{NR}$ & $N R$ & $N R$ & $N R$ & NR \\
\hline $\mathrm{NO} 2$ & 0.53 & 5.31 & 0.73 & 0.89 & 0.21 & 0.12 & 0.02 \\
\hline $\mathrm{NO} 3$ & 0.86 & 3.77 & 1.08 & 0.98 & 0.31 & 0.35 & 0.03 \\
\hline EPA 8493 & $N R$ & $\mathrm{NR}$ & $\mathrm{NR}$ & $N R$ & $N R$ & $\mathrm{NR}$ & $N R$ \\
\hline NO6 & 0.52 & 2.29 & 0.93 & 1.08 & 0.24 & 0.20 & 0.02 \\
\hline EPA 8910 & $\mathrm{NR}$ & $\mathrm{NR}$ & $\mathrm{NR}$ & $N R$ & $N R$ & $\mathrm{NR}$ & NR \\
\hline NO7A & 0.97 & 3.28 & 0.97 & 1.06 & 0.44 & 0.72 & 0.06 \\
\hline NO7BC & 0.63 & 6.72 & 0.67 & 0.54 & 0.15 & 0.16 & 0.02 \\
\hline EPA 8912 & $\mathrm{NR}$ & NR & $N R$ & $\mathrm{NR}$ & $N R$ & $\mathrm{NR}$ & NR \\
\hline NO8BC & 1.73 & 4.10 & 0.96 & 1.47 & 0.30 & 0.40 & 0.04 \\
\hline $\mathrm{NO9}$ & 0.84 & 8.07 & 0.67 & 0.79 & 0.20 & 0.19 & 0.02 \\
\hline EPA 8911 & $N R$ & NR & $\mathrm{NR}$ & $\mathrm{NR}$ & $N R$ & $\mathrm{NR}$ & NR \\
\hline NO10 & 0.85 & 4.26 & 1.16 & 1.48 & 0.27 & 0.15 & 0.03 \\
\hline $\mathrm{NO} 11 \mathrm{AB}$ & 1.34 & 3.90 & 1.67 & 1.02 & 0.42 & 0.73 & 0.04 \\
\hline $\mathrm{NO} 11 \mathrm{C}$ & 1.62 & 4.49 & 2.90 & 0.68 & 0.32 & 0.64 & 0.04 \\
\hline US Mean soil & $\mathrm{NR}$ & $\mathrm{NR}$ & $\mathrm{NR}$ & $\mathrm{NR}$ & $N R$ & $\mathrm{NR}$ & NR \\
\hline Netherlands & $N R$ & $\mathrm{NR}$ & $\mathrm{NR}$ & $N R$ & $N R$ & $\mathrm{NR}$ & NR \\
\hline LDEQ & $\mathrm{NR}$ & $\mathrm{NR}$ & $\mathrm{NR}$ & $\mathrm{NR}$ & $N R$ & $\mathrm{NR}$ & NR \\
\hline
\end{tabular}




\begin{tabular}{|c|c|c|c|c|c|c|c|}
\hline Field No. & $\begin{array}{c}\text { Loss on ignition } \\
\text { wt. } \% \\
\text { WDXRF } \\
\end{array}$ & $\begin{array}{c}\mathrm{CO} 2 \\
\%\end{array}$ & $\begin{array}{c}\text { Carbonate C } \\
\%\end{array}$ & $\begin{array}{l}\mathbf{S} \\
\%\end{array}$ & $\begin{array}{c}\mathrm{Ag} \\
\mathrm{ppm} \\
\mathrm{ICP}-\mathrm{MS}\end{array}$ & $\begin{array}{c}\text { Al } \\
\text { ppm } \\
\text { ICP-MS }\end{array}$ & $\begin{array}{c}\text { As } \\
\text { ppm } \\
\text { Hydride }\end{array}$ \\
\hline Chalmette 1 & 32.9 & 0.22 & 0.06 & 1.81 & $<2$ & 58500 & 8.2 \\
\hline Chalmette 1d & 32.9 & 0.22 & 0.06 & 1.80 & $<2$ & 59800 & 8.2 \\
\hline EPA 8939* & NR & $\mathrm{NR}$ & NR & $\mathrm{NR}$ & 0.663 & 9860 & 9.7 \\
\hline Chalmette 2 & 18.5 & 0.68 & 0.19 & 0.76 & $<2$ & 72600 & 9.9 \\
\hline Chalmette 2d & 18.5 & 0.71 & 0.19 & 0.77 & $<2$ & 72900 & 10 \\
\hline EPA $10174^{* *}$ & $\mathrm{NR}$ & $\mathrm{NR}$ & $\mathrm{NR}$ & $N R$ & 0.4 & 8930 & 18 \\
\hline $\mathrm{NO} 1$ & 38.2 & NA & $\mathrm{NA}$ & $\mathrm{NA}$ & $<3$ & 32100 & NA \\
\hline EPA 8490 & $\mathrm{NR}$ & $\mathrm{NR}$ & $\mathrm{NR}$ & $N R$ & 0.036 & 3820 & $\mathrm{NR}$ \\
\hline $\mathrm{NO} 2$ & 6.19 & NA & $\mathrm{NA}$ & NA & $<3$ & 21500 & NA \\
\hline $\mathrm{NO} 3$ & 24.3 & $\mathrm{NA}$ & $\mathrm{NA}$ & $\mathrm{NA}$ & $<3$ & 27500 & $\mathrm{NA}$ \\
\hline EPA 8493 & $N R$ & $\mathrm{NR}$ & $N R$ & $N R$ & 0.6 & 3250 & $\mathrm{NR}$ \\
\hline $\mathrm{NO6}$ & 8.81 & $\mathrm{NA}$ & $\mathrm{NA}$ & $\mathrm{NA}$ & $<3$ & 23000 & $\mathrm{NA}$ \\
\hline EPA 8910 & $\mathrm{NR}$ & $\mathrm{NR}$ & $N R$ & $N R$ & $N R$ & 5090 & $N R$ \\
\hline NO7A & 22.3 & NA & $\mathrm{NA}$ & $\mathrm{NA}$ & $<3$ & 37000 & $N A$ \\
\hline NO7BC & 11.1 & NA & $\mathrm{NA}$ & $\mathrm{NA}$ & $<3$ & 12800 & $\mathrm{NA}$ \\
\hline EPA 8912 & $\mathrm{NR}$ & $\mathrm{NR}$ & $N R$ & $N R$ & 0.12 & 4650 & NR \\
\hline NO8BC & 12.2 & NA & $\mathrm{NA}$ & $\mathrm{NA}$ & $<3$ & 29000 & NA \\
\hline NO9 & 11.5 & NA & $\mathrm{NA}$ & $\mathrm{NA}$ & $<3$ & 20200 & NA \\
\hline EPA 8911 & $\mathrm{NR}$ & $\mathrm{NR}$ & $\mathrm{NR}$ & $N R$ & $\mathrm{NR}$ & 1850 & $\mathrm{NR}$ \\
\hline NO10 & 7.89 & $\mathrm{NR}$ & $\mathrm{NR}$ & $N R$ & $<3$ & 33800 & NA \\
\hline N011AB & 43.5 & $\mathrm{NR}$ & $\mathrm{NR}$ & $N R$ & 4.4 & 34100 & $\mathrm{NA}$ \\
\hline $\mathrm{NO} 11 \mathrm{C}$ & 38.4 & $\mathrm{NR}$ & $\mathrm{NR}$ & NR & 3.3 & 27400 & $N A$ \\
\hline US Mean soil & $\mathrm{NR}$ & $\mathrm{NR}$ & $\mathrm{NR}$ & 0.12 & $\mathrm{NR}$ & 47000 & 5.2 \\
\hline Netherlands & $\mathrm{NR}$ & $\mathrm{NR}$ & $\mathrm{NR}$ & $\mathrm{NR}$ & $\mathrm{NR}$ & $\mathrm{NR}$ & 30 \\
\hline LDEQ & $\mathrm{NR}$ & $\mathrm{NR}$ & $\mathrm{NR}$ & $\mathrm{NR}$ & 39 & $\mathrm{NR}$ & 12 \\
\hline
\end{tabular}




\begin{tabular}{|c|c|c|c|c|c|c|c|}
\hline Chalmette 1 & 9.2 & 367 & 1.7 & 0.3 & 6190 & 0.6 & 50 \\
\hline Chalmette 1d & 8.8 & 363 & 1.6 & 0.2 & 6170 & 0.6 & 45 \\
\hline EPA 8939* & 9.7 & 668.7 & 0.8 & $\mathrm{NR}$ & 6700 & 0.3 & NR \\
\hline Chalmette 2 & 11 & 676 & 2 & 0.3 & 8630 & 1.0 & 64 \\
\hline Chalmette 2d & 11 & 689 & 1.9 & 0.3 & 9080 & 1 & 65 \\
\hline EPA $10174^{* *}$ & 18.3 & 156 & 0.8 & $\mathrm{NR}$ & 5730 & 1.8 & NR \\
\hline NO1 & 24 & 593 & 1.0 & 0.8 & 36900 & 30 & 45 \\
\hline EPA 8490 & 2.69 & 68.1 & 0.22 & $\mathrm{NR}$ & 9000 & 0.3 & NR \\
\hline $\mathrm{NO} 2$ & 4.5 & 415 & 0.8 & 0.2 & 42500 & 1.9 & 32 \\
\hline NO3 & 22 & 496 & 1.0 & 1.3 & 29800 & 18 & 40 \\
\hline $\mathrm{NO5}$ & 38 & 630 & 1.1 & 0.9 & 40900 & 22 & 35 \\
\hline EPA 8493 & 14 & 344 & 0.3 & $\mathrm{NR}$ & 33600 & 5.5 & NR \\
\hline NO6 & 7.3 & 457 & 0.7 & 0.3 & 17300 & 2.0 & 34 \\
\hline EPA 8910 & 19.2 & 222 & 0.41 & $\mathrm{NR}$ & 17800 & 6.4 & NR \\
\hline NO7A & 37 & 1150 & 1.8 & 1.9 & 25300 & 3.7 & 49 \\
\hline NO7BC & 5.8 & 398 & 0.5 & 0.2 & 51200 & 2.1 & 25 \\
\hline EPA 8912 & 28.9 & 216 & 0.41 & $\mathrm{NR}$ & 19300 & 6.8 & NR \\
\hline NO8BC & 114 & 653 & 0.8 & 0.4 & 31800 & 2.0 & 35 \\
\hline NO9 & 6.1 & 544 & 0.7 & 0.2 & 65900 & 1.1 & 30 \\
\hline EPA 8911 & 4.38 & 42.8 & 0.15 & $\mathrm{NR}$ & 20900 & 0.3 & NR \\
\hline NO10 & 5.2 & 564 & 1.0 & 0.1 & 33400 & 0.6 & 37 \\
\hline $\mathrm{NO} 11 \mathrm{AB}$ & 43 & 625 & 1.2 & 1.1 & 29400 & 46 & 45 \\
\hline N011C & 36 & 552 & 0.9 & 0.8 & 36600 & 43 & 41 \\
\hline US Mean soil & 5.2 & 440 & 0.63 & 0.56 & 9200 & 0.06 & 75 \\
\hline Netherlands & 30 & 400 & $\mathrm{NR}$ & $\mathrm{NR}$ & $N R$ & 5 & NR \\
\hline LDEQ & 12 & 550 & 16 & $\mathrm{NR}$ & $\mathrm{NR}$ & 3.9 & NR \\
\hline
\end{tabular}




\begin{tabular}{|c|c|c|c|c|c|c|c|}
\hline Chalmette 1 & 10 & 63 & 5 & 31 & 28400 & 14 & 0.1 \\
\hline Chalmette 1d & 10 & 60 & 5 & 29 & 28600 & 14 & 0.1 \\
\hline EPA 8939* & 6.98 & 17.2 & $\mathrm{NR}$ & 42.2 & 15900 & NR & 0.08 \\
\hline Chalmette 2 & 11 & 75 & 6 & 41 & 33800 & 16 & 0.1 \\
\hline Chalmette 2d & 12 & 77 & 6 & 41 & 34800 & 17 & 0.1 \\
\hline EPA $10174^{* *}$ & 6.37 & 25 & $\mathrm{NR}$ & 41.9 & 15600 & NR & 0.12 \\
\hline $\mathrm{NO} 1$ & 27 & 81 & 2.7 & 230 & 23000 & 6.8 & 0.6 \\
\hline EPA 8490 & 3.71 & 12.9 & $\mathrm{NR}$ & 55.5 & 6520 & NR & 0.17 \\
\hline $\mathrm{NO} 2$ & 40 & 27 & 0.9 & 69 & 13500 & 3.6 & 0.08 \\
\hline $\mathrm{NO} 3$ & 70 & 57 & 2.2 & 150 & 19300 & 5.5 & 1.7 \\
\hline $\mathrm{NO5}$ & 30 & 67 & 1.7 & 267 & 18600 & 4.8 & 1.4 \\
\hline EPA 8493 & 3.34 & 28.7 & $\mathrm{NR}$ & 101 & 9830 & $\mathrm{NR}$ & 0.56 \\
\hline NO6 & 74 & 24 & 1.1 & 45 & 10800 & 4.6 & 0.3 \\
\hline EPA 8910 & 4.15 & 28.7 & $\mathrm{NR}$ & 126 & 12800 & $\mathrm{NR}$ & 1.07 \\
\hline NO7A & 47 & 57 & 2.6 & 141 & 39400 & 7.8 & 2.8 \\
\hline NO7BC & 79 & 22 & 0.6 & 87 & 10900 & 2.5 & 4.1 \\
\hline EPA 8912 & 5.5 & 29.5 & $\mathrm{NR}$ & 143 & 13100 & $\mathrm{NR}$ & 0.53 \\
\hline NO8BC & 75 & 43 & 1.2 & 52 & 17700 & 5.5 & 2.2 \\
\hline NO9 & 42 & 38 & 1.1 & 54 & 12100 & 3.8 & 0.1 \\
\hline EPA 8911 & 3.26 & 8.15 & $\mathrm{NR}$ & 23.8 & 5350 & $\mathrm{NR}$ & 0.062 \\
\hline NO10 & 65 & 29 & 1.4 & 22 & 11100 & 6.2 & 0.1 \\
\hline $\mathrm{NO} 11 \mathrm{AB}$ & 24 & 118 & 3.3 & 418 & 29800 & 7.1 & 2.0 \\
\hline $\mathrm{NO11C}$ & 42 & 93 & 2.6 & 333 & 27600 & 5.8 & 1.5 \\
\hline US Mean soil & 9.1 & 54 & 6 & 25 & 26000 & 17 & 0.058 \\
\hline Netherlands & 50 & 250 & $\mathrm{NR}$ & 100 & $N R$ & NR & 2 \\
\hline LDEQ & 470 & Total Cr NR & $\mathrm{NR}$ & 310 & $\mathrm{NR}$ & $\mathrm{NR}$ & 2.3 \\
\hline
\end{tabular}




\begin{tabular}{|c|c|c|c|c|c|c|c|}
\hline Chalmette 1 & 14800 & 28 & 35 & 12200 & 499 & 3.6 & 20700 \\
\hline Chalmette 1d & 14700 & 25 & 34 & 12400 & 493 & 3.4 & 20900 \\
\hline EPA 8939* & 3530 & $N R$ & $\mathrm{NR}$ & 7920 & 516 & NR & 20400 \\
\hline Chalmette 2 & 18200 & 34 & 40 & 12700 & 770 & 2.0 & 14700 \\
\hline Chalmette 2d & 19200 & 35 & 40 & 13100 & 792 & 1.9 & 15000 \\
\hline EPA $10174^{* *}$ & 2840 & $N R$ & $\mathrm{NR}$ & 6310 & 244 & $\mathrm{NR}$ & 9480 \\
\hline $\mathrm{NO1}$ & 10200 & 20 & 19 & 8720 & 275 & 4.38 & 14200 \\
\hline EPA 8490 & 453 & NR & $N R$ & 1530 & 216 & $\mathrm{NR}$ & 1240 \\
\hline $\mathrm{NO} 2$ & 8100 & 15 & 10 & 3480 & 143 & 1.8 & 6160 \\
\hline $\mathrm{NO} 3$ & 9460 & 19 & 17 & 5830 & 259 & 2.5 & 8640 \\
\hline EPA 8493 & $\mathrm{NR}$ & $N R$ & $\mathrm{NR}$ & 4120 & 385 & $\mathrm{NR}$ & 3130 \\
\hline NO6 & 9380 & 16 & 8.7 & 3260 & 166 & 1.5 & 7060 \\
\hline EPA 8910 & 1570 & $N R$ & $\mathrm{NR}$ & 4670 & 239 & $\mathrm{NR}$ & 5820 \\
\hline NO7A & 9690 & 23 & 32 & 5800 & 513 & 3.0 & 7670 \\
\hline NO7BC & 4850 & 11 & 8.4 & 4010 & 153 & 1.7 & 5340 \\
\hline EPA 8912 & 1750 & $\mathrm{NR}$ & $\mathrm{NR}$ & 6200 & 200 & NR & 12200 \\
\hline NO8BC & 13600 & 17 & 12 & 11600 & 339 & 2.4 & 8010 \\
\hline NO9 & 7580 & 14 & 11 & 5650 & 188 & 1.5 & 5870 \\
\hline EPA 8911 & 456 & $N R$ & $\mathrm{NR}$ & 2710 & 83.7 & $\mathrm{NR}$ & 2020 \\
\hline $\mathrm{NO10}$ & 13400 & 18 & 13 & 5560 & 219 & 1.5 & 9380 \\
\hline N011AB & 9890 & 22 & 20 & 8850 & 347 & 4.0 & 11500 \\
\hline $\mathrm{NO11C}$ & 9430 & 18 & 15 & 10700 & 338 & 3.6 & 28200 \\
\hline US Mean soil & 15000 & 37 & 24 & 9000 & 550 & 0.97 & 12000 \\
\hline Netherlands & $\mathrm{NR}$ & $\mathrm{NR}$ & $\mathrm{NR}$ & $\mathrm{NR}$ & $N R$ & 40 & NR \\
\hline LDEQ & $\mathrm{NR}$ & $\mathrm{NR}$ & $\mathrm{NR}$ & $\mathrm{NR}$ & $N R$ & $\mathrm{NR}$ & NR \\
\hline
\end{tabular}




\begin{tabular}{|c|c|c|c|c|c|c|c|}
\hline Chalmette 1 & 12 & 32 & 959 & 44 & 80 & 0.7 & 1.4 \\
\hline Chalmette 1d & 9.8 & 32 & 947 & 43 & 81 & 0.7 & 1.4 \\
\hline EPA 8939* & $\mathrm{NR}$ & 27.2 & $\mathrm{NR}$ & 34.8 & $N R$ & NR & NR \\
\hline Chalmette 2 & 15 & 31 & 820 & 51 & 96 & 1.0 & 0.8 \\
\hline Chalmette 2d & 16 & 32 & 867 & 52 & 98 & 1.0 & 0.8 \\
\hline EPA $10174^{* *}$ & $\mathrm{NR}$ & 18 & $\mathrm{NR}$ & 36.8 & $N R$ & $\mathrm{NR}$ & NR \\
\hline $\mathrm{NO1}$ & 6.4 & 35 & 2100 & 939 & 45 & 6.7 & NA \\
\hline EPA 8490 & $\mathrm{NR}$ & 7.3 & NR & 28.2 & NR & 0.54 & NR \\
\hline $\mathrm{NO} 2$ & $<2$ & 14 & 356 & 102 & 28 & 1.9 & NA \\
\hline $\mathrm{NO} 3$ & 6.9 & 23 & 1660 & 402 & 37 & 8.3 & NA \\
\hline EPA 8493 & $\mathrm{NR}$ & 14.2 & $\mathrm{NR}$ & 655 & $N R$ & $\mathrm{NR}$ & NR \\
\hline NO6 & $<2$ & 14 & 732 & 497 & 33 & 2.5 & NA \\
\hline EPA 8910 & $\mathrm{NR}$ & 21 & $\mathrm{NR}$ & 597 & $N R$ & $N R$ & $\mathrm{NR}$ \\
\hline NO7A & 10.2 & 31 & 3490 & 2180 & 43 & 12 & NA \\
\hline NO7BC & $<2$ & 16 & 621 & 207 & 17 & 1.3 & $\mathrm{NA}$ \\
\hline EPA 8912 & $\mathrm{NR}$ & 28.8 & $\mathrm{NR}$ & 627 & $N R$ & $\mathrm{NR}$ & $\mathrm{NR}$ \\
\hline NO8BC & 5.3 & 21 & 1840 & 760 & 46 & 4.3 & NA \\
\hline NO9 & $<2$ & 19 & 851 & 290 & 26 & 4.5 & NA \\
\hline EPA 8911 & $N R$ & 10.2 & $\mathrm{NR}$ & 71.7 & $N R$ & $\mathrm{NR}$ & NR \\
\hline NO10 & 3.6 & 16 & 561 & 96 & 45 & 2.4 & $\mathrm{NA}$ \\
\hline N011AB & 7.1 & 38 & 3580 & 754 & 46 & 11 & $\mathrm{NA}$ \\
\hline $\mathrm{NO11C}$ & 5.9 & 37 & 3380 & 557 & 39 & 9.6 & NA \\
\hline US Mean soil & 11 & 19 & 430 & 19 & 67 & 0.66 & 0.26 \\
\hline Netherlands & $\mathrm{NR}$ & 100 & $\mathrm{NR}$ & 150 & $N R$ & NR & NR \\
\hline LDEQ & $\mathrm{NR}$ & 160 & $\mathrm{NR}$ & 400 & $N R$ & 3.1 & 39 \\
\hline
\end{tabular}




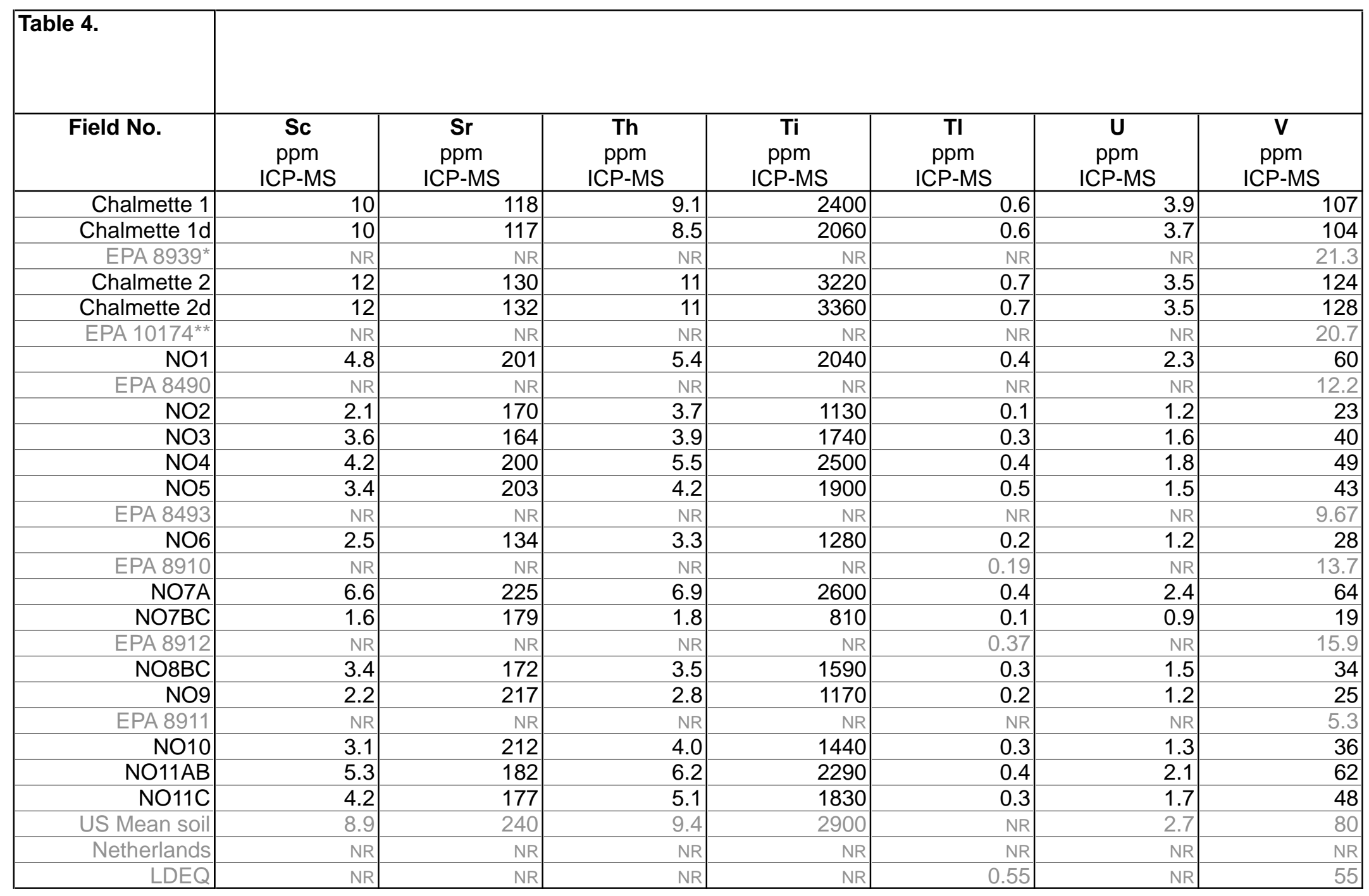




\begin{tabular}{|c|c|c|}
\hline Table 4. & & \\
\hline Field No. & $\begin{array}{c}\mathbf{Y} \\
\text { ppm } \\
\text { ICP-MS }\end{array}$ & $\begin{array}{c}\mathbf{Z n} \\
\text { ppm } \\
\text { ICP-MS }\end{array}$ \\
\hline Chalmette 1 & 19 & 174 \\
\hline Chalmette 1d & 18 & 174 \\
\hline EPA 8939* & NR & 153 \\
\hline Chalmette 2 & 24 & 435 \\
\hline Chalmette 2d & 25 & 454 \\
\hline EPA 10174** & $\mathrm{NR}$ & 650 \\
\hline $\mathrm{NO} 1$ & 15 & 5500 \\
\hline EPA 8490 & NR & 274 \\
\hline NO2 & 8.4 & 755 \\
\hline $\mathrm{NO3}$ & 13 & 3830 \\
\hline $\mathrm{NO} 4$ & 15 & 2880 \\
\hline NO5 & 10 & 7880 \\
\hline EPA 8493 & NR & 1830 \\
\hline $\mathrm{NO6}$ & 10 & 694 \\
\hline EPA 8910 & NR & 2160 \\
\hline NO7A & 20 & 1740 \\
\hline NO7BC & 6.4 & 863 \\
\hline EPA 8912 & NR & 2820 \\
\hline NO8BC & 12 & 849 \\
\hline NO9 & 8.9 & 432 \\
\hline EPA 8911 & NR & 291 \\
\hline NO10 & 13 & 209 \\
\hline $\mathrm{NO} 11 \mathrm{AB}$ & 17 & 9680 \\
\hline $\mathrm{NO} 11 \mathrm{C}$ & 14 & 9700 \\
\hline US Mean soil & 25 & 60 \\
\hline Netherlands & NR & 500 \\
\hline LDEQ & $\mathrm{NR}$ & 2300 \\
\hline
\end{tabular}




\begin{tabular}{|c|c|c|c|c|c|c|c|c|}
\hline $\begin{array}{l}\text { Table 5a. Blank } \\
\text { and leachate } \\
\text { concentrations. }\end{array}$ & \multicolumn{8}{|c|}{$\begin{array}{l}\text { Analytical results for simulated gastric fluid leach tests performed on flood sediment samples. Table } 5 \text { a reports } \\
\text { concentrations in the leachate, table } 5 \mathrm{~b} \text { reports values recalculated to mg leached per kg solid. Analyst: Suzette Morman. } \\
\text { Contact: Geoff Plumlee, gplumlee@usgs.gov. The Chalmette samples originally contained approximately } 60-75 \% \text { water, } \\
\text { so measured concentrations on the Chalmette leachates would increase proportionally if recalculated to dry weight. The } \\
\text { NO samples were dried prior to leaching. This test is modified slightly from a simulated gastric fluid leach test used by } \\
\text { EPA to assess lead bioaccessibility from soils (Chris Weis, oral communications, } 2004 \text { ). One part solid is added to } 100 \\
\text { parts simulated gastric fluid ( } \mathrm{pH} 1.5 \text {, composed of } \mathrm{HCl} \text { and glycine), mixture rotated for } 2 \text { hours at } 37^{\circ} \mathrm{C} \text {, Concentrations } \\
\text { below the detection limit are shown by }(<) \text {. Separate batches, each using a new blank leachate solution, are separated by } \\
\text { horizontal borders in the database. All analyses by ICP-MS except } \mathrm{Hg} \text { by cold vapor atomic fluorescence. }\end{array}$} \\
\hline Field No. & $\begin{array}{c}\mathbf{A g} \\
\mathrm{ug} / \mathrm{L}\end{array}$ & $\begin{array}{c}\text { Al } \\
\mathrm{ug} / \mathrm{L}\end{array}$ & $\begin{array}{c}\text { As } \\
\mathrm{ug} / \mathrm{L}\end{array}$ & $\begin{array}{c}\mathbf{B a} \\
\mathrm{ug} / \mathrm{L}\end{array}$ & $\begin{array}{c}\mathrm{Be} \\
\mathrm{ug} / \mathrm{L}\end{array}$ & $\begin{array}{c}\mathbf{B i} \\
u g / L \\
\end{array}$ & $\begin{array}{c}\mathbf{C a} \\
\mathrm{mg} / \mathrm{L}\end{array}$ & $\begin{array}{c}\text { Cd } \\
\mathrm{ug} / \mathrm{L}\end{array}$ \\
\hline blank & $<3$ & 30 & 1 & 9.2 & $<0.05$ & 0.9 & 0.3 & 0.1 \\
\hline Blank dup & $<3$ & 31 & $<1$ & 11 & $<0.05$ & 0.9 & 0.7 & 0.08 \\
\hline Chalmette 1 & $<3$ & 5850 & 19 & 305 & 3.0 & 0.6 & 38 & 3.6 \\
\hline Chalmette 1 dup & $<3$ & 6620 & 22 & 348 & 3.3 & 0.5 & 44 & 4.2 \\
\hline Chalmette 2 & $<3$ & 5140 & 35 & 540 & 3.1 & 0.6 & 53 & 7.7 \\
\hline Chalmette 2 dup & $<3$ & 4730 & 33 & 511 & 2.7 & 0.6 & 49 & 6.6 \\
\hline NO 7C & $<3$ & 2240 & 9.0 & 475 & 0.3 & 0.5 & 287 & 7.0 \\
\hline NO 7C dup & $<3$ & 3550 & 15 & 553 & 0.8 & 0.5 & 426 & 11 \\
\hline Blank & $<3$ & 7.9 & 2 & 49 & $<0.05$ & 0.2 & 0.5 & $<0.02$ \\
\hline NO1 & $<3$ & 16500 & 138 & 1240 & 2.4 & $<0.2$ & 305 & 180 \\
\hline NO2 & $<3$ & 7500 & 17 & 615 & 0.7 & $<0.2$ & 353 & 10 \\
\hline NO2 dup & $<3$ & 7910 & 16 & 581 & 0.8 & $<0.2$ & 357 & 11 \\
\hline NO3 & $<3$ & 16600 & 121 & 1380 & 2.2 & $<0.2$ & 266 & 122 \\
\hline NO4 & $<3$ & 15200 & 114 & 1560 & 2.4 & $<0.2$ & 285 & 64 \\
\hline NO5 & $<3$ & 16900 & 177 & 1420 & 2.1 & $<0.2$ & 390 & 93 \\
\hline NO6 & $<3$ & 13900 & 68 & 1500 & 1.5 & 0.2 & 154 & 20 \\
\hline NO7 A & $<3$ & 22900 & 241 & 3180 & 4.0 & 2.8 & 221 & 36 \\
\hline NO7 BC & $<3$ & 10100 & 40 & 1690 & 1.0 & $<0.2$ & 476 & 16 \\
\hline NO8 BC & $<3$ & 11800 & 336 & 1500 & 1.1 & 0.8 & 204 & 9.0 \\
\hline NO9 & $<3$ & 14800 & 32 & 1680 & 1.3 & 0.4 & 545 & 8.4 \\
\hline NO10 & $<3$ & 11600 & 26 & 1250 & 1.3 & 0.2 & 245 & 4.9 \\
\hline NO11 & $<3$ & 36200 & 245 & 1880 & 3.5 & $<0.2$ & 364 & 328 \\
\hline NO11 C & $<3$ & 30100 & 192 & 1400 & 2.8 & $<0.2$ & 362 & 290 \\
\hline
\end{tabular}




\begin{tabular}{|c|c|c|c|c|c|c|c|c|}
\hline $\begin{array}{l}\text { Table 5a. Blank } \\
\text { and leachate } \\
\text { concentrations. }\end{array}$ & & & & & & & & \\
\hline Field No. & $\begin{array}{c}\mathbf{C e} \\
\mathrm{uq} / \mathrm{L}\end{array}$ & $\begin{array}{c}\text { Co } \\
\text { Uq/L }\end{array}$ & $\begin{array}{c}\mathbf{C r} \\
\mathrm{uq} / \mathrm{L}\end{array}$ & $\begin{array}{c}\text { Cs } \\
\mathrm{ug} / \mathrm{L}\end{array}$ & $\begin{array}{c}\mathrm{Cu} \\
\mathrm{uq} / \mathrm{L}\end{array}$ & $\begin{array}{c}\text { Dy } \\
\text { uq/L }\end{array}$ & $\begin{array}{c}\text { Er } \\
u g / L\end{array}$ & $\begin{array}{c}\mathrm{Eu} \\
\mathrm{ug} / \mathrm{L}\end{array}$ \\
\hline blank & 0.07 & 0.08 & 68 & 0.04 & 4.2 & $<0.005$ & 0.01 & $<0.005$ \\
\hline Blank dup & 0.1 & 0.1 & 68 & 0.03 & 4.1 & $<0.005$ & 0.03 & $<0.005$ \\
\hline Chalmette 1 & 50 & 13 & 74 & 0.4 & 31 & 2.8 & 1.3 & 0.7 \\
\hline Chalmette 1 dup & 52 & 14 & 73 & 0.4 & 31 & 3.1 & 1.4 & 0.8 \\
\hline Chalmette 2 & 110 & 19 & 74 & 0.3 & 133 & 9.3 & 4.6 & 2.4 \\
\hline Chalmette 2 dup & 102 & 17 & 76 & 0.3 & 121 & 8.4 & 4.1 & 2.2 \\
\hline NO 7C & 15 & 4.1 & 77 & 0.1 & 101 & 1.4 & 0.7 & 0.3 \\
\hline NO 7C dup & 34 & 6.2 & 84 & 0.1 & 216 & 2.5 & 1.2 & 0.6 \\
\hline Blank & 0.07 & 0.07 & 71 & $<0.02$ & 1.1 & 0.02 & 0.04 & 0.008 \\
\hline $\mathrm{NO1}$ & 51 & 170 & 180 & 1.3 & 21 & 3.9 & 2.3 & 0.9 \\
\hline $\mathrm{NO} 2$ & 35 & 322 & 114 & 0.5 & 536 & 3.2 & 1.7 & 0.8 \\
\hline NO2 dup & 35 & 306 & 120 & 0.5 & 688 & 3.2 & 1.7 & 0.8 \\
\hline NO3 & 47 & 600 & 194 & 2.1 & 36 & 3.9 & 2.2 & 0.9 \\
\hline NO4 & 66 & 145 & 160 & 0.8 & 307 & 5.8 & 3.1 & 1.5 \\
\hline NO5 & 38 & 253 & 223 & 0.8 & 4.2 & 2.9 & 1.8 & 0.7 \\
\hline NO6 & 58 & 762 & 119 & 0.8 & 341 & 5.3 & 2.8 & 1.3 \\
\hline N07 A & 75 & 366 & 138 & 1.1 & 891 & 7.6 & 4.0 & 1.9 \\
\hline NO7 BC & 40 & 704 & 115 & 0.6 & 159 & 3.3 & 1.8 & 0.9 \\
\hline NO8 BC & 37 & 614 & 120 & 0.7 & 253 & 3.7 & 2.1 & 0.9 \\
\hline NO9 & 44 & 348 & 207 & 1.5 & 390 & 4.4 & 2.4 & 1.1 \\
\hline NO10 & 65 & 560 & 127 & 0.7 & 133 & 6.8 & 3.5 & 1.6 \\
\hline NO11 & 67 & 178 & 421 & 2.1 & 143 & 4.9 & 2.8 & 1.3 \\
\hline NO11 C & 54 & 304 & 378 & 1.9 & 324 & 4.2 & 2.5 & 1.0 \\
\hline
\end{tabular}




\begin{tabular}{|c|c|c|c|c|c|c|c|c|}
\hline $\begin{array}{l}\text { Table 5a. Blank } \\
\text { and leachate } \\
\text { concentrations. }\end{array}$ & & & & & & & & \\
\hline Field No. & $\begin{array}{c}\text { Fe } \\
\mathrm{uq} / \mathrm{L}\end{array}$ & $\begin{array}{c}\text { Ga } \\
\mathrm{ug} / \mathrm{L}\end{array}$ & $\begin{array}{c}\text { Gd } \\
u q / L\end{array}$ & $\begin{array}{c}\text { Ge } \\
u q / L\end{array}$ & $\begin{array}{c}\text { Ho } \\
\text { uq/L }\end{array}$ & $\begin{array}{c}\mathbf{K} \\
\mathrm{mq} / \mathrm{L}\end{array}$ & $\begin{array}{c}\text { La } \\
\mathrm{ug} / \mathrm{L}\end{array}$ & $\begin{array}{c}\mathbf{L i} \\
\mathrm{uq} / \mathrm{L}\end{array}$ \\
\hline blank & $<50$ & $<0.05$ & $<0.005$ & $<0.05$ & $<0.005$ & 0.07 & 0.02 & $<0.9$ \\
\hline Blank dup & $<50$ & $<0.05$ & 0.02 & $<0.05$ & $<0.005$ & 0.05 & 0.05 & $<0.9$ \\
\hline Chalmette 1 & 11200 & 1.4 & 3.8 & $<0.05$ & 0.5 & 10 & 29 & $<0.9$ \\
\hline Chalmette 1 dup & 12600 & 1.4 & 4.2 & $<0.05$ & 0.6 & 12 & 32 & $<0.9$ \\
\hline Chalmette 2 & 28500 & 2 & 12 & $<0.05$ & 1.8 & 10.0 & 50 & $<0.9$ \\
\hline Chalmette 2 dup & 26500 & 1.9 & 11 & $<0.05$ & 1.7 & 9.0 & 47 & $<0.9$ \\
\hline NO 7C & 5820 & 0.6 & 1.8 & $<0.05$ & 0.3 & 2.0 & 9.1 & $<0.9$ \\
\hline NO 7C dup & 9080 & 0.9 & 2.9 & $<0.05$ & 0.5 & 3.0 & 18 & $<0.9$ \\
\hline Blank & $<50$ & 0.1 & 0.005 & $<0.05$ & $<0.005$ & $<0.03$ & 0.06 & 0.6 \\
\hline $\mathrm{NO1}$ & 56400 & 2.6 & 4.7 & $<0.05$ & 0.8 & 13 & 28 & 16 \\
\hline $\mathrm{NO} 2$ & 13000 & 1.6 & 4.4 & $<0.05$ & 0.6 & 1.8 & 21 & 5.6 \\
\hline NO2 dup & 13000 & 1.7 & 4.2 & $<0.05$ & 0.6 & 1.8 & 21 & 5.8 \\
\hline NO3 & 42200 & 2.9 & 4.6 & 0.4 & 0.8 & 9.3 & 27 & 12 \\
\hline NO4 & 27400 & 2.6 & 7.2 & 0.6 & 1.1 & 5.2 & 37 & 9.5 \\
\hline NO5 & 74400 & 3.2 & 3.2 & 0.2 & 0.6 & 15.3 & 23 & 14 \\
\hline NO6 & 18000 & 3.2 & 6.6 & 0.5 & 1.0 & 7.7 & 30 & 10 \\
\hline N07 A & 29200 & 4.4 & 9.2 & 1.4 & 1.4 & 11 & 40 & 21 \\
\hline NO7 BC & 15300 & 2.0 & 4.0 & 0.08 & 0.6 & 5.5 & 22 & 8.2 \\
\hline NO8 BC & 8160 & 2.3 & 4.4 & 0.4 & 0.7 & 7.0 & 21 & 5.9 \\
\hline NO9 & 16700 & 3.5 & 5.5 & 0.4 & 0.9 & 4.8 & 25 & 14 \\
\hline NO10 & 14000 & 3.1 & 8.6 & 0.4 & 1.3 & 5.9 & 33 & 8.4 \\
\hline NO11 & 114000 & 4.2 & 5.9 & 0.5 & 1.0 & 19 & 38 & 22 \\
\hline NO11 C & 102000 & 4.4 & 5.0 & 0.9 & 0.9 & 25 & 29 & 25 \\
\hline
\end{tabular}




\begin{tabular}{|c|c|c|c|c|c|c|c|c|}
\hline $\begin{array}{l}\text { Table 5a. Blank } \\
\text { and leachate } \\
\text { concentrations. }\end{array}$ & & & & & & & & \\
\hline Field No. & $\begin{array}{c}\mathrm{Lu} \\
\mathrm{ug} / \mathrm{L}\end{array}$ & $\begin{array}{c}\mathrm{Mg} \\
\mathrm{mg} / \mathrm{L}\end{array}$ & $\begin{array}{c}\mathrm{Mn} \\
\mathrm{ug} / \mathrm{L}\end{array}$ & $\begin{array}{c}\text { Mo } \\
\mathrm{ug} / \mathrm{L}\end{array}$ & $\begin{array}{c}\mathrm{Na} \\
\mathrm{mg} / \mathrm{L}\end{array}$ & $\begin{array}{c}\mathrm{Nb} \\
\mathrm{ug} / \mathrm{L}\end{array}$ & $\begin{array}{c}\mathrm{Nd} \\
\mathrm{ug} / \mathrm{L}\end{array}$ & $\begin{array}{c}\mathrm{Ni} \\
\mathrm{ug} / \mathrm{L}\end{array}$ \\
\hline blank & $<0.1$ & 0.02 & 1.3 & 5.1 & $<0.5$ & 2.6 & 0.2 & 1.7 \\
\hline Blank dup & $<0.1$ & 0.04 & 2.0 & 2.6 & $<0.5$ & 2.1 & 0.2 & 1.8 \\
\hline Chalmette 1 & 0.1 & 46 & 2010 & $<2$ & 139 & 2.2 & 22 & 48 \\
\hline Chalmette 1 dup & 0.1 & 52 & 2340 & $<2$ & 157 & 1.8 & 24 & 53 \\
\hline Chalmette 2 & 0.4 & 33 & 4270 & $<2$ & 92 & 1.6 & 58 & 49 \\
\hline Chalmette 2 dup & 0.4 & 30 & 3790 & $<2$ & 84 & 1.4 & 53 & 44 \\
\hline NO 7C & $<0.1$ & 14 & 452 & $<2$ & 14 & 1.3 & 8.3 & 32 \\
\hline NO 7C dup & 0.1 & 24 & 686 & $<2$ & 22 & 1.1 & 14 & 36 \\
\hline Blank & $<0.1$ & 0.3 & 1.1 & $<2$ & 0.1 & $<0.2$ & 0.1 & 1.4 \\
\hline NO1 & 0.2 & 39 & 1400 & $<2$ & 75 & 0.24 & 20 & 120 \\
\hline $\mathrm{NO} 2$ & 0.2 & 15 & 469 & $<2$ & 3.5 & $<0.2$ & 18 & 35 \\
\hline NO2 dup & 0.2 & 16 & 493 & $<2$ & 3.6 & $<0.2$ & 18 & 34 \\
\hline NO3 & 0.2 & 30 & 1410 & $<2$ & 39 & $<0.2$ & 21 & 91 \\
\hline NO4 & 0.4 & 26 & 1570 & $<2$ & 15 & $<0.2$ & 31 & 72 \\
\hline NO5 & 0.2 & 63 & 3420 & $<2$ & 142 & $<0.2$ & 16 & 118 \\
\hline NO6 & 0.3 & 19 & 1020 & $<2$ & 23 & $<0.2$ & 29 & 50 \\
\hline N07 A & 0.4 & 24 & 3490 & $<2$ & 45 & $<0.2$ & 38 & 72 \\
\hline NO7 BC & 0.2 & 29 & 886 & $<2$ & 22 & $<0.2$ & 18 & 50 \\
\hline NO8 BC & 0.2 & 60 & 884 & $<2$ & 17 & $<0.2$ & 19 & 41 \\
\hline NO9 & 0.3 & 35 & 995 & $<2$ & 11 & $<0.2$ & 24 & 71 \\
\hline NO10 & 0.4 & 29 & 1190 & $<2$ & 9.8 & $<0.2$ & 36 & 57 \\
\hline NO11 & 0.3 & 72 & 2850 & $<2$ & 124 & 0.4 & 28 & 167 \\
\hline NO11 C & 0.3 & 87 & 2590 & $<2$ & 273 & 0.3 & 25 & 133 \\
\hline
\end{tabular}




\begin{tabular}{|c|c|c|c|c|c|c|c|c|}
\hline $\begin{array}{l}\text { Table 5a. Blank } \\
\text { and leachate } \\
\text { concentrations. }\end{array}$ & & & & & & & & \\
\hline Field No. & $\begin{array}{c}\mathbf{P} \\
\mathrm{mg} / \mathrm{L}\end{array}$ & $\begin{array}{c}\mathbf{P b} \\
\mathrm{ug} / \mathrm{L}\end{array}$ & $\begin{array}{c}\mathrm{Pr} \\
\mathrm{ug} / \mathrm{L}\end{array}$ & $\begin{array}{c}\mathbf{R b} \\
\mathrm{ug} / \mathrm{L}\end{array}$ & $\begin{array}{c}\mathrm{Sb} \\
\mathrm{ug} / \mathrm{L}\end{array}$ & $\begin{array}{c}\mathrm{Sc} \\
\mathrm{ug} / \mathrm{L}\end{array}$ & $\begin{array}{c}\mathrm{Se} \\
\mathrm{ug} / \mathrm{L}\end{array}$ & $\begin{array}{l}\mathrm{SiO2} \\
\mathrm{mg} / \mathrm{L}\end{array}$ \\
\hline blank & 0.05 & 4.8 & 0.01 & 0.06 & 6.6 & 0.8 & 1.8 & 13 \\
\hline Blank dup & 0.04 & 6.7 & $<0.01$ & 0.04 & 4.4 & 1 & 1.4 & 13 \\
\hline Chalmette 1 & 1.9 & 179 & 5.8 & 13 & 4.3 & 1.5 & 3.5 & 19 \\
\hline Chalmette 1 dup & 2.2 & 204 & 6.1 & 14 & 3.4 & 1.7 & 5.6 & 19 \\
\hline Chalmette 2 & 2.6 & 242 & 13 & 11 & 3.2 & 2 & 4.1 & 21 \\
\hline Chalmette 2 dup & 2.4 & 220 & 13 & 10.0 & 2.8 & 2 & 3.4 & 21 \\
\hline NO 7C & 2.4 & 1120 & 2.0 & 2.7 & 3.1 & 1.7 & 2.4 & 17 \\
\hline NO 7C dup & 3.4 & 1820 & 3.8 & 4.1 & 3.7 & 1.9 & 2.1 & 19 \\
\hline Blank & 0.02 & 1.2 & $<0.01$ & 0.03 & 1.3 & 0.9 & 1.2 & 17 \\
\hline $\mathrm{NO1}$ & 8.6 & 4370 & 5.1 & 26 & 2.9 & 3.5 & 6.1 & 38 \\
\hline $\mathrm{NO} 2$ & 2 & 637 & 4.3 & 6.6 & 5.1 & 2.9 & 2 & 33 \\
\hline NO2 dup & 2.1 & 615 & 4.3 & 6.7 & 4.3 & 2.9 & 2.4 & 34 \\
\hline NO3 & 11 & 2390 & 5.2 & 24 & 1.0 & 3.1 & 4.5 & 38 \\
\hline NO4 & 7.4 & 8150 & 7.6 & 15 & 5.4 & 4.4 & 3.6 & 54 \\
\hline NO5 & 27 & 2090 & 4.1 & 22 & 1.4 & 3.5 & 10 & 44 \\
\hline NO6 & 6.1 & 4770 & 6.8 & 23 & 4.3 & 2.5 & 3.6 & 32 \\
\hline N07 A & 27 & 19000 & 8.9 & 29 & 8.6 & 3.5 & 4.3 & 41 \\
\hline NO7 BC & 4.6 & 1720 & 4.6 & 14 & 2.4 & 2.9 & 2.8 & 35 \\
\hline NO8 BC & 13 & 5840 & 4.5 & 19 & 4.9 & 2.3 & 4.2 & 28 \\
\hline NO9 & 6.5 & 2320 & 5.6 & 19 & 3.8 & 4.7 & 3.2 & 54 \\
\hline NO10 & 4.3 & 763 & 8.2 & 19 & 4.6 & 3 & 3 & 32 \\
\hline NO11 & 23 & 3330 & 7.3 & 33 & 6.1 & 7.8 & 6 & 71 \\
\hline NO11 C & 19 & 2910 & 6.1 & 33 & 5.8 & 6.9 & 6.5 & 63 \\
\hline
\end{tabular}




\begin{tabular}{|c|c|c|c|c|c|c|c|c|}
\hline $\begin{array}{l}\text { Table 5a. Blank } \\
\text { and leachate } \\
\text { concentrations. }\end{array}$ & & & & & & & & \\
\hline Field No. & $\begin{array}{c}\mathrm{Sm} \\
\mathrm{ug} / \mathrm{L}\end{array}$ & $\begin{array}{c}\mathrm{SO} 4 \\
\mathrm{mg} / \mathrm{L}\end{array}$ & $\begin{array}{c}\mathrm{Sr} \\
\mathrm{ug} / \mathrm{L}\end{array}$ & $\begin{array}{c}\mathrm{Ta} \\
\mathrm{ug} / \mathrm{L}\end{array}$ & $\begin{array}{c}\mathrm{Tb} \\
\mathrm{ug} / \mathrm{L}\end{array}$ & $\begin{array}{c}\text { Th } \\
\mathrm{ug} / \mathrm{L}\end{array}$ & $\begin{array}{c}\mathrm{Ti} \\
\mathrm{ug} / \mathrm{L}\end{array}$ & $\begin{array}{c}\mathrm{TI} \\
\mathrm{ug} / \mathrm{L}\end{array}$ \\
\hline blank & $<0.01$ & 10 & 1.0 & 1.1 & $<0.005$ & $<0.2$ & 9.1 & 0.1 \\
\hline Blank dup & $<0.01$ & 10 & 1.8 & 0.9 & $<0.005$ & $<0.2$ & 11 & $<0.1$ \\
\hline Chalmette 1 & 3.5 & 22 & 457 & 1.0 & 0.5 & $<0.2$ & 24 & 0.1 \\
\hline Chalmette 1 dup & 3.7 & 22 & 518 & 0.7 & 0.5 & $<0.2$ & 23 & 0.2 \\
\hline Chalmette 2 & 11.6 & 22 & 381 & 0.7 & 1.6 & 0.4 & 45 & 0.1 \\
\hline Chalmette 2 dup & 9.7 & 20 & 345 & 0.6 & 1.5 & 0.4 & 48 & 0.1 \\
\hline NO 7C & 1.6 & 15 & 625 & 0.6 & 0.3 & $<0.2$ & 48 & $<0.1$ \\
\hline NO 7C dup & 2.4 & 14 & 942 & 0.5 & 0.4 & $<0.2$ & 58 & 0.1 \\
\hline Blank & $<0.01$ & $<2$ & 2.0 & $<0.02$ & $<0.005$ & $<0.2$ & 33 & $<0.1$ \\
\hline $\mathrm{NO1}$ & 3.4 & 63 & 1080 & 0.07 & 0.7 & 0.34 & 68 & 0.8 \\
\hline $\mathrm{NO} 2$ & 3.7 & 7 & 596 & 0.05 & 0.6 & $<0.2$ & 188 & 0.1 \\
\hline NO2 dup & 3.7 & 8 & 597 & 0.03 & 0.6 & $<0.2$ & 188 & 0.1 \\
\hline NO3 & 3.6 & 141 & 787 & 0.09 & 0.6 & $<0.2$ & 65 & 0.63 \\
\hline NO4 & 6.0 & 29 & 742 & $<0.02$ & 1.0 & $<0.2$ & 94 & 0.3 \\
\hline NO5 & 2.5 & 545 & 1400 & 0.05 & 0.5 & 0.74 & 44 & 1.9 \\
\hline NO6 & 5.8 & 24 & 490 & $<0.02$ & 1.0 & $<0.2$ & 74 & 0.3 \\
\hline N07 A & 7.7 & 30 & 908 & 0.1 & 1.3 & $<0.2$ & 70 & 0.7 \\
\hline NO7 BC & 3.4 & 27 & 1190 & $<0.02$ & 0.6 & $<0.2$ & 100 & 0.3 \\
\hline NO8 BC & 3.8 & 14 & 501 & $<0.02$ & 0.6 & $<0.2$ & 91 & 0.2 \\
\hline NO9 & 4.9 & 24 & 1180 & $<0.02$ & 0.8 & $<0.2$ & 251 & 0.2 \\
\hline NO10 & 7.5 & 11 & 649 & 0.1 & 1.2 & $<0.2$ & 88 & 0.2 \\
\hline NO11 & 4.8 & 35 & 1190 & 0.2 & 0.8 & 0.3 & 71 & 0.8 \\
\hline NO11 C & 4.1 & 81 & 1130 & 0.1 & 0.7 & 0.2 & 79 & 0.6 \\
\hline
\end{tabular}




\begin{tabular}{|c|c|c|c|c|c|c|c|c|}
\hline $\begin{array}{l}\text { Table 5a. Blank } \\
\text { and leachate } \\
\text { concentrations. }\end{array}$ & & & & & & & & \\
\hline Field No. & $\begin{array}{c}\mathrm{Tm} \\
\mathrm{ug} / \mathrm{L}\end{array}$ & $\begin{array}{c}\mathbf{U} \\
\mathrm{ug} / \mathrm{L}\end{array}$ & $\begin{array}{c}\text { V } \\
\mathrm{ug} / \mathrm{L}\end{array}$ & $\begin{array}{c}W \\
\mathrm{ug} / \mathrm{L}\end{array}$ & $\begin{array}{c}Y \\
\mathrm{ug} / \mathrm{L}\end{array}$ & $\begin{array}{c}\mathbf{Y b} \\
\mathrm{ug} / \mathrm{L}\end{array}$ & $\begin{array}{c}\mathrm{Zn} \\
\mathrm{ug} / \mathrm{L}\end{array}$ & $\begin{array}{c}\mathrm{Zr} \\
\mathrm{ug} / \mathrm{L}\end{array}$ \\
\hline blank & $<0.005$ & $<0.1$ & 17 & 2.1 & $<0.01$ & $<0.005$ & 24 & $<0.2$ \\
\hline Blank dup & $<0.005$ & $<0.1$ & 16 & 1.9 & 0.02 & 0.005 & 32 & $<0.2$ \\
\hline Chalmette 1 & 0.2 & 1.3 & 79 & 1.9 & 19 & 0.8 & 727 & 1.2 \\
\hline Chalmette 1 dup & 0.2 & 1.3 & 88 & 1.5 & 20 & 0.9 & 804 & 1.2 \\
\hline Chalmette 2 & 0.6 & 4.8 & 87 & 1.3 & 51 & 3.0 & 2560 & 1.7 \\
\hline Chalmette 2 dup & 0.5 & 4.6 & 82 & 1.3 & 47 & 2.9 & 2300 & 1.6 \\
\hline NO 7C & 0.1 & 1.0 & 28 & 1.2 & 8.0 & 0.6 & 2980 & 0.6 \\
\hline NO 7C dup & 0.2 & 1.4 & 35 & 1.0 & 14 & 0.8 & 4690 & 0.8 \\
\hline Blank & $<0.005$ & $<0.1$ & 16 & $<0.5$ & $<0.01$ & $<0.005$ & 18 & $<0.2$ \\
\hline $\mathrm{NO1}$ & 0.3 & 3.5 & 105 & 2.7 & 28 & 1.7 & 39800 & 8.3 \\
\hline $\mathrm{NO} 2$ & 0.2 & 2.6 & 40 & 29 & 18 & 1.3 & 5220 & 2.8 \\
\hline NO2 dup & 0.2 & 2.5 & 43 & 28 & 18 & 1.3 & 5200 & 2.9 \\
\hline NO3 & 0.3 & 2.8 & 86 & 12 & 26 & 1.7 & 34100 & 6 \\
\hline NO4 & 0.4 & 3.3 & 73 & 4.6 & 37 & 2.4 & 25000 & 5.6 \\
\hline NO5 & 0.2 & 2.1 & 105 & 5.0 & 20 & 1.3 & 73400 & 11 \\
\hline NO6 & 0.4 & 2.8 & 63 & 32.7 & 30 & 2.1 & 7290 & 3.5 \\
\hline N07 A & 0.5 & 3.5 & 135 & 9.3 & 41 & 3.0 & 14100 & 4.2 \\
\hline NO7 BC & 0.2 & 2.6 & 54 & 37 & 18 & 1.4 & 7210 & 3.7 \\
\hline NO8 BC & 0.3 & 3.3 & 50 & 48 & 22 & 1.6 & 4010 & 2.3 \\
\hline NO9 & 0.3 & 4.5 & 73 & 54 & 26 & 1.9 & 3240 & 4.2 \\
\hline NO10 & 0.4 & 3.3 & 56 & 26 & 35 & 2.6 & 1550 & 2.4 \\
\hline NO11 & 0.4 & 2.8 & 156 & 3.7 & 33 & 2.1 & 84500 & 6.8 \\
\hline $\mathrm{NO} 11 \mathrm{C}$ & 0.3 & 2.2 & 132 & 5.0 & 28 & 1.8 & 84700 & 5.3 \\
\hline
\end{tabular}




\begin{tabular}{|l|r|}
\hline $\begin{array}{l}\text { Table 5a. Blank } \\
\text { and leachate } \\
\text { concentrations. }\end{array}$ \\
\\
\\
\\
\\
\\
\hline \multicolumn{1}{|c|}{ Field No. } & Hg \\
\hline blank & ng/L \\
\hline Blank dup & $\mathrm{NA}$ \\
\hline Chalmette 1 & $\mathrm{NA}$ \\
\hline Chalmette 1 dup & $\mathrm{NA}$ \\
\hline Chalmette 2 & $\mathrm{NA}$ \\
\hline Chalmette 2 dup & $\mathrm{NA}$ \\
\hline NO 7C & $\mathrm{NA}$ \\
\hline NO 7C dup & $\mathrm{NA}$ \\
\hline Blank & $\mathrm{NA}$ \\
\hline NO1 & $\mathrm{NA}$ \\
\hline NO2 & $\mathrm{NA}$ \\
\hline NO2 dup & $\mathrm{NA}$ \\
\hline NO3 & $\mathrm{NA}$ \\
\hline NO4 & $\mathrm{NA}$ \\
\hline NO5 & $\mathrm{NA}$ \\
\hline NO6 & $\mathrm{NA}$ \\
\hline NO7 A & $\mathrm{NA}$ \\
\hline NO7 BC \\
\hline NO8 BC \\
\hline NO9 \\
\hline NO10 \\
\hline NO11 \\
\hline NO11 C \\
\hline
\end{tabular}




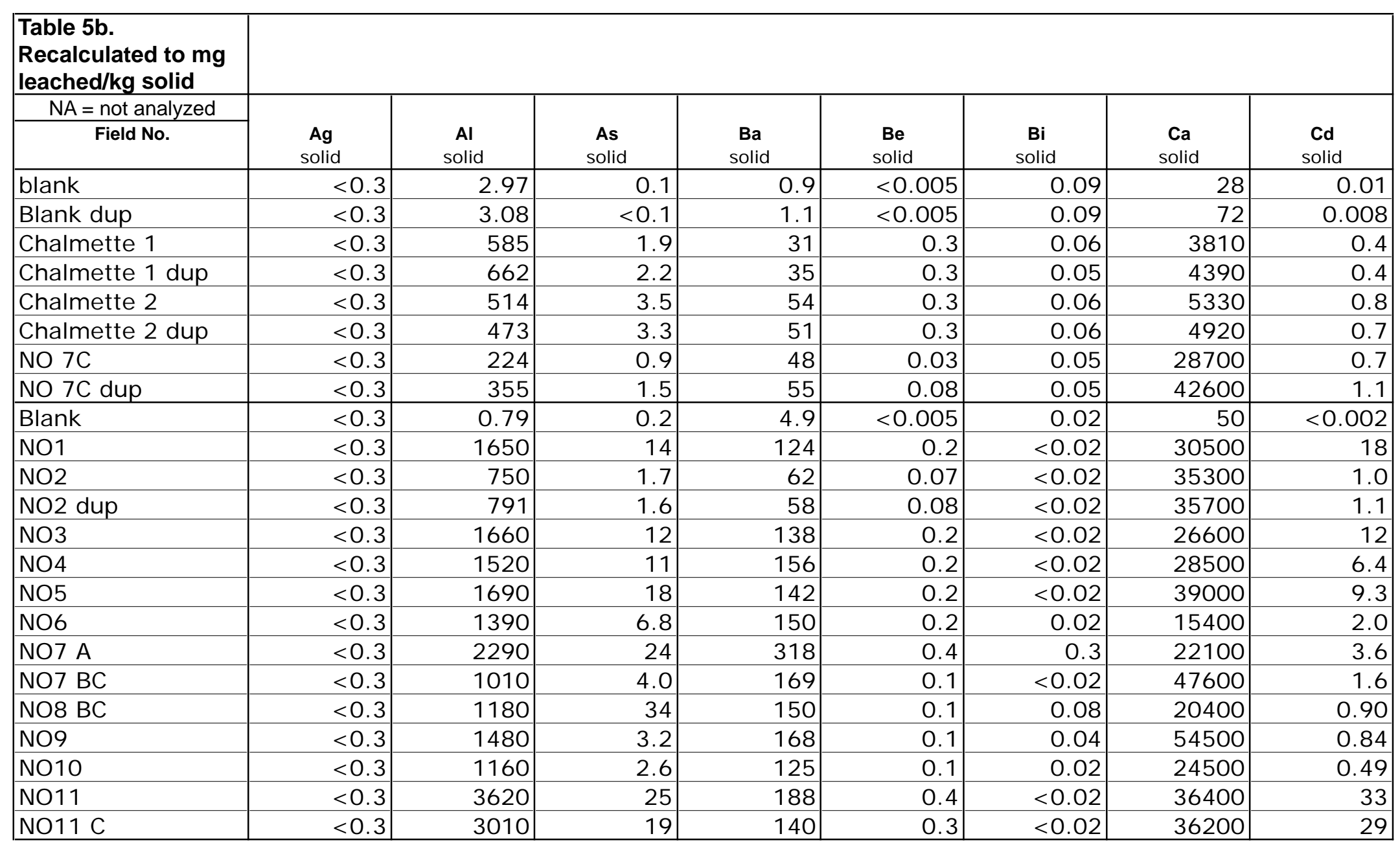




\begin{tabular}{|c|c|c|c|c|c|c|c|c|}
\hline $\begin{array}{l}\text { Table } 5 \mathrm{~b} . \\
\text { Recalculated to } \mathrm{mg} \\
\text { leached/kg solid }\end{array}$ & & \multirow{2}{*}{$\begin{array}{c}\text { Co } \\
\text { solid }\end{array}$} & \multirow{2}{*}{$\begin{array}{c}\mathrm{Cr} \\
\text { solid }\end{array}$} & \multirow{2}{*}{$\begin{array}{c}\text { Cs } \\
\text { solid }\end{array}$} & \multirow{2}{*}{$\begin{array}{c}\mathrm{Cu} \\
\text { solid }\end{array}$} & \multirow{2}{*}{$\begin{array}{c}\text { Dy } \\
\text { solid }\end{array}$} & \multirow{2}{*}{$\begin{array}{c}\text { Er } \\
\text { solid }\end{array}$} & \multirow{2}{*}{$\begin{array}{l}\text { Eu } \\
\text { solid }\end{array}$} \\
\hline $\begin{array}{c}\mathrm{NA}=\text { not analyzed } \\
\text { Field No. }\end{array}$ & $\begin{array}{c}\mathrm{Ce} \\
\text { solid } \\
\end{array}$ & & & & & & & \\
\hline blank & 0.007 & 0.008 & 6.8 & 0.004 & 0.4 & $<0.0005$ & 0.001 & $<0.0005$ \\
\hline Blank dup & 0.01 & 0.01 & 6.8 & 0.003 & 0.4 & $<0.0005$ & 0.003 & $<0.0005$ \\
\hline Chalmette 1 & 5.0 & 1.3 & 7.4 & 0.04 & 3.1 & 0.3 & 0.1 & 0.07 \\
\hline Chalmette 1 dup & 5.2 & 1.4 & 7.3 & 0.04 & 3.1 & 0.3 & 0.1 & 0.08 \\
\hline Chalmette 2 & 11 & 1.9 & 7.4 & 0.03 & 13 & 0.9 & 0.5 & 0.2 \\
\hline Chalmette 2 dup & 10 & 1.7 & 7.6 & 0.03 & 12 & 0.8 & 0.41 & 0.2 \\
\hline NO 7C & 1.5 & 0.4 & 7.7 & 0.01 & 10 & 0.1 & 0.07 & 0.03 \\
\hline NO 7C dup & 3.4 & 0.6 & 8.4 & 0.01 & 22 & 0.2 & 0.1 & 0.06 \\
\hline Blank & 0.007 & 0.007 & 7.1 & $<0.002$ & 0.1 & 0.002 & 0.004 & 0.0008 \\
\hline $\mathrm{NO1}$ & 5.1 & 17 & 18 & 0.1 & 2.1 & 0.4 & 0.2 & 0.09 \\
\hline NO2 dup & 3.5 & 31 & 12 & 0.05 & 69 & 0.3 & 0.2 & 0.08 \\
\hline NO3 & 4.7 & 60 & 19 & 0.2 & 3.6 & 0.4 & 0.2 & 0.09 \\
\hline NO4 & 6.6 & 15 & 16 & 0.08 & 31 & 0.6 & 0.3 & 0.1 \\
\hline NO5 & 3.8 & 25 & 22 & 0.08 & 0.4 & 0.3 & 0.2 & 0.07 \\
\hline NO6 & 5.8 & 76 & 12 & 0.08 & 34 & 0.5 & 0.3 & 0.1 \\
\hline N07 A & 7.5 & 37 & 14 & 0.1 & 89 & 0.8 & 0.4 & 0.2 \\
\hline NO7 BC & 4 & 70 & 12 & 0.06 & 16 & 0.3 & 0.2 & 0.09 \\
\hline NO8 BC & 3.7 & 61 & 12 & 0.07 & 25 & 0.4 & 0.2 & 0.09 \\
\hline NO9 & 4.4 & 35 & 21 & 0.1 & 39 & 0.4 & 0.2 & 0.1 \\
\hline NO10 & 6.5 & 56 & 13 & 0.07 & 13 & 0.7 & 0.3 & 0.2 \\
\hline NO11 & 6.7 & 18 & 42 & 0.2 & 14 & 0.5 & 0.3 & 0.1 \\
\hline $\mathrm{NO11C}$ & 5.4 & 30 & 38 & 0.2 & 32 & 0.4 & 0.2 & 0.1 \\
\hline
\end{tabular}




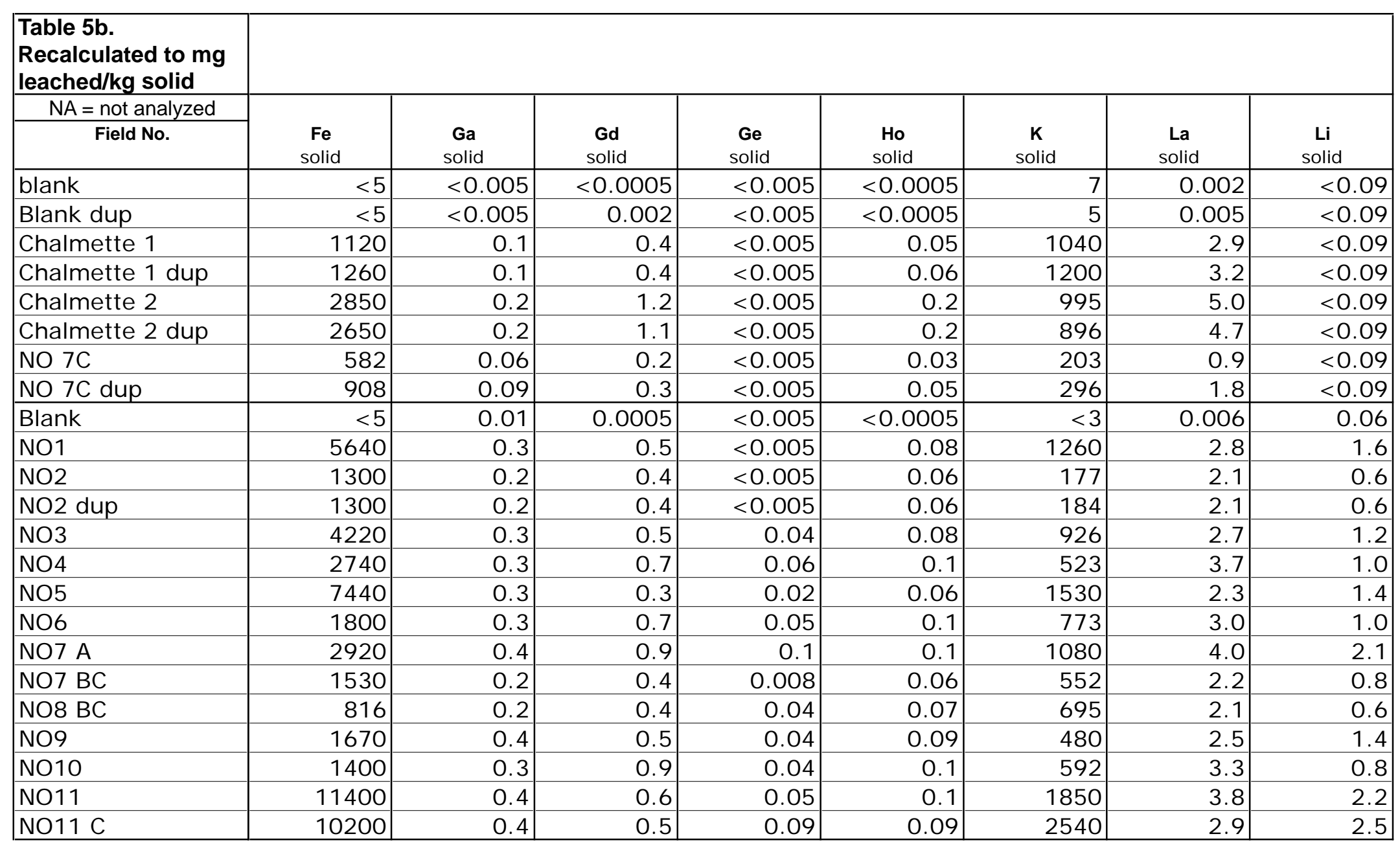




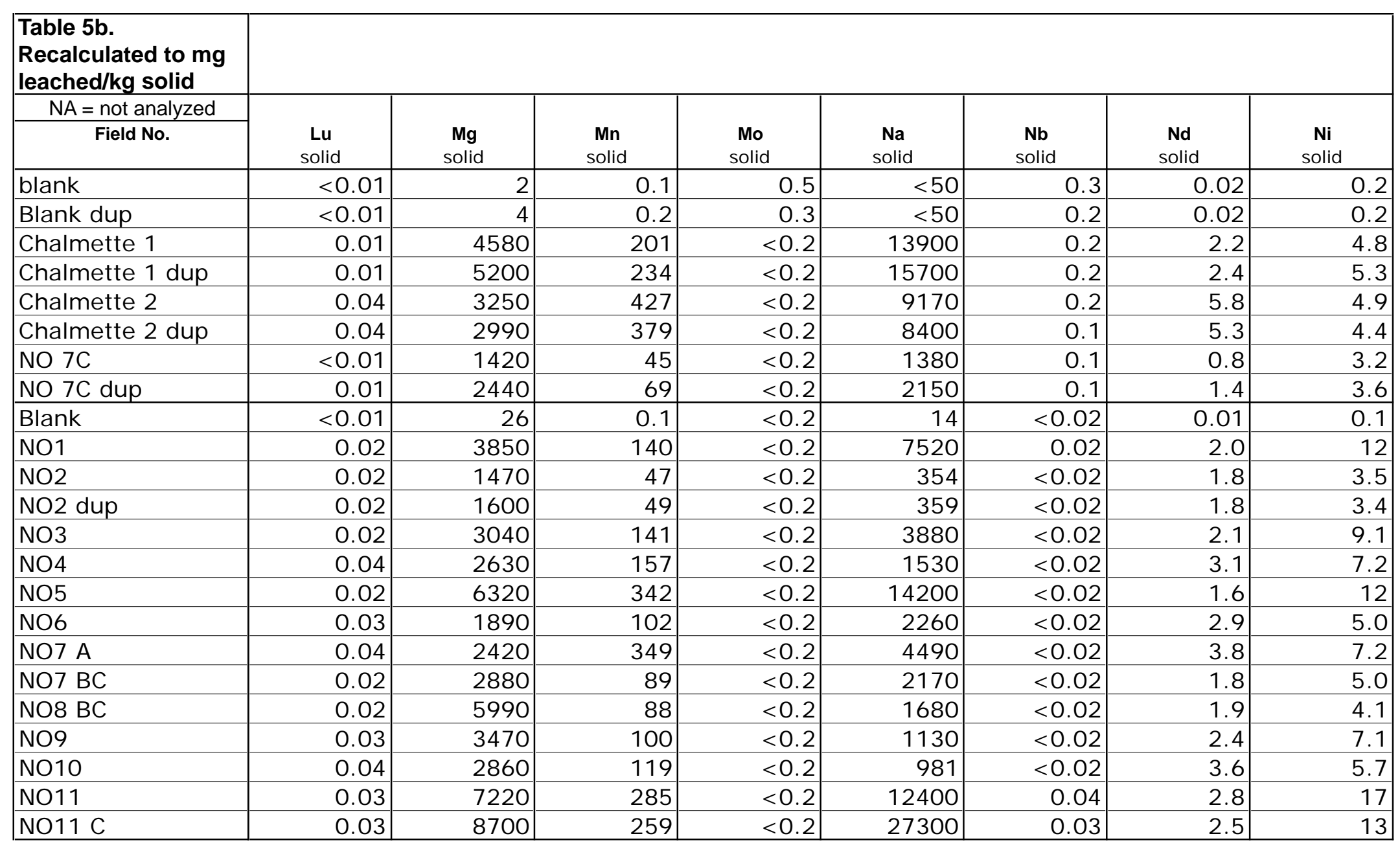




\begin{tabular}{|c|c|c|c|c|c|c|c|c|}
\hline $\begin{array}{l}\text { Table } 5 \mathrm{~b} . \\
\text { Recalculated to } \mathrm{mg} \\
\text { leached/kg solid }\end{array}$ & & \multirow[b]{3}{*}{$\begin{array}{c}\mathbf{P b} \\
\text { solid }\end{array}$} & \multirow[b]{3}{*}{$\begin{array}{c}\mathrm{Pr} \\
\text { solid } \\
\end{array}$} & \multirow[b]{3}{*}{$\begin{array}{c}\mathbf{R b} \\
\text { solid } \\
\end{array}$} & \multirow[b]{3}{*}{$\begin{array}{c}\text { Sb } \\
\text { solid }\end{array}$} & \multirow[b]{3}{*}{$\begin{array}{c}\text { Sc } \\
\text { solid }\end{array}$} & \multirow[b]{3}{*}{$\begin{array}{c}\text { Se } \\
\text { solid }\end{array}$} & \multirow[b]{3}{*}{$\begin{array}{l}\text { SiO2 } \\
\text { solid } \\
\end{array}$} \\
\hline \multirow{2}{*}{$\frac{\mathrm{NA}=\text { not analyzed }}{\text { Field No. }}$} & \multirow[b]{2}{*}{$\begin{array}{c}\mathbf{P} \\
\text { solid }\end{array}$} & & & & & & & \\
\hline & & & & & & & & \\
\hline blank & 5 & 0.5 & 0.001 & 0.006 & 0.7 & 0.08 & 0.2 & 1280 \\
\hline Blank dup & 4 & 0.7 & $<0.001$ & 0.004 & 0.4 & 0.1 & 0.1 & 1260 \\
\hline Chalmette 1 & 190 & 18 & 0.6 & 1.3 & 0.4 & 0.2 & 0.4 & 1910 \\
\hline Chalmette 1 dup & 220 & 20 & 0.6 & 1.4 & 0.3 & 0.2 & 0.6 & 1940 \\
\hline Chalmette 2 & 260 & 24 & 1.3 & 1.1 & 0.3 & 0.2 & 0.4 & 2130 \\
\hline Chalmette 2 dup & 240 & 22 & 1.3 & 1.0 & 0.3 & 0.2 & 0.3 & 2090 \\
\hline NO 7C & 240 & 112 & 0.2 & 0.3 & 0.3 & 0.2 & 0.2 & 1660 \\
\hline NO 7C dup & 340 & 182 & 0.4 & 0.4 & 0.4 & 0.2 & 0.2 & 1930 \\
\hline Blank & 2 & 0.1 & $<0.001$ & 0.003 & 0.1 & 0.09 & 0.1 & 1650 \\
\hline $\mathrm{NO1}$ & 860 & 437 & 0.5 & 2.6 & 0.3 & 0.4 & 0.6 & 3780 \\
\hline NO2 dup & 210 & 62 & 0.4 & 0.7 & 0.4 & 0.3 & 0.2 & 3360 \\
\hline NO3 & 1140 & 239 & 0.5 & 2.4 & 0.1 & 0.3 & 0.5 & 3840 \\
\hline $\mathrm{NO} 4$ & 740 & 815 & 0.8 & 1.5 & 0.5 & 0.4 & 0.4 & 5440 \\
\hline NO5 & 2660 & 209 & 0.4 & 2.2 & 0.1 & 0.4 & 1 & 4380 \\
\hline NO6 & 610 & 477 & 0.7 & 2.3 & 0.4 & 0.3 & 0.4 & 3150 \\
\hline N07 A & 2740 & 1900 & 0.9 & 2.9 & 0.9 & 0.4 & 0.4 & 4090 \\
\hline NO7 BC & 460 & 172 & 0.5 & 1.4 & 0.2 & 0.3 & 0.3 & 3500 \\
\hline NO8 BC & 1280 & 584 & 0.4 & 1.9 & 0.5 & 0.2 & 0.4 & 2760 \\
\hline NO9 & 650 & 232 & 0.6 & 1.9 & 0.4 & 0.5 & 0.3 & 5410 \\
\hline NO10 & 430 & 76.3 & 0.8 & 1.9 & 0.5 & 0.3 & 0.3 & 3220 \\
\hline NO11 & 2290 & 333 & 0.7 & 3.3 & 0.6 & 0.8 & 0.6 & 7110 \\
\hline $\mathrm{NO11C}$ & 1860 & 291 & 0.6 & 3.3 & 0.6 & 0.7 & 0.7 & 6320 \\
\hline
\end{tabular}




\begin{tabular}{|c|c|c|c|c|c|c|c|c|}
\hline $\begin{array}{l}\text { Table } 5 \mathrm{~b} . \\
\text { Recalculated to } \mathrm{mg} \\
\text { leached/kg solid }\end{array}$ & & \multirow{2}{*}{$\begin{array}{l}\mathrm{SO} 4 \\
\text { solid }\end{array}$} & \multirow{2}{*}{$\begin{array}{c}\text { Sr } \\
\text { solid }\end{array}$} & \multirow{2}{*}{$\begin{array}{c}\text { Ta } \\
\text { solid }\end{array}$} & \multirow{2}{*}{$\begin{array}{l}\text { Tb } \\
\text { solid }\end{array}$} & \multirow{2}{*}{$\begin{array}{l}\text { Th } \\
\text { solid }\end{array}$} & \multirow{2}{*}{$\begin{array}{c}\mathrm{Ti} \\
\text { solid }\end{array}$} & \multirow{2}{*}{$\begin{array}{c}\text { TI } \\
\text { solid }\end{array}$} \\
\hline $\begin{array}{c}\mathrm{NA}=\text { not analyzed } \\
\text { Field No. }\end{array}$ & $\begin{array}{l}\text { Sm } \\
\text { solid }\end{array}$ & & & & & & & \\
\hline blank & $<0.001$ & 1000 & 0.1 & 0.11 & $<0.0005$ & $<0.02$ & 0.9 & 0.01 \\
\hline Blank dup & $<0.001$ & 1000 & 0.2 & 0.1 & $<0.0005$ & $<0.02$ & 1.1 & $<0.01$ \\
\hline Chalmette 1 & 0.4 & 2200 & 46 & 0.1 & 0.05 & $<0.02$ & 2.4 & 0.01 \\
\hline Chalmette 1 dup & 0.4 & 2200 & 52 & 0.1 & 0.05 & $<0.02$ & 2.3 & 0.02 \\
\hline Chalmette 2 & 1.2 & 2200 & 38 & 0.1 & 0.2 & 0.04 & 4.5 & 0.01 \\
\hline Chalmette 2 dup & 1.0 & 2000 & 35 & 0.1 & 0.1 & 0.04 & 4.8 & 0.01 \\
\hline NO 7C & 0.2 & 1500 & 63 & 0.1 & 0.03 & $<0.02$ & 4.8 & $<0.01$ \\
\hline NO 7C dup & 0.2 & 1400 & 94 & 0.0 & 0.04 & $<0.02$ & 5.8 & 0.01 \\
\hline Blank & $<0.001$ & $<200$ & 0.2 & $<0.002$ & $<0.0005$ & $<0.02$ & 3.3 & $<0.01$ \\
\hline NO1 & 0.3 & 6300 & 108 & 0.007 & 0.07 & 0.03 & 6.8 & 0.08 \\
\hline NO2 dup & 0.4 & 800 & 60 & 0.003 & 0.06 & $<0.02$ & 19 & 0.01 \\
\hline NO3 & 0.4 & 14100 & 79 & 0.009 & 0.06 & $<0.02$ & 6.5 & 0.06 \\
\hline NO4 & 0.6 & 2900 & 74 & $<0.002$ & 0.1 & $<0.02$ & 9.4 & 0.03 \\
\hline NO5 & 0.3 & 54500 & 140 & 0.005 & 0.05 & 0.07 & 4.4 & 0.2 \\
\hline NO6 & 0.6 & 2400 & 49 & $<0.002$ & 0.1 & $<0.02$ & 7.4 & 0.03 \\
\hline NO7 A & 0.8 & 3000 & 91 & 0.01 & 0.1 & $<0.02$ & 7.0 & 0.07 \\
\hline NO7 BC & 0.3 & 2700 & 119 & $<0.002$ & 0.06 & $<0.02$ & 10 & 0.03 \\
\hline NO8 BC & 0.4 & 1400 & 50 & $<0.002$ & 0.06 & $<0.02$ & 9.1 & 0.02 \\
\hline NO9 & 0.5 & 2400 & 118 & $<0.002$ & 0.08 & $<0.02$ & 25 & 0.02 \\
\hline NO10 & 0.7 & 1100 & 65 & 0.01 & 0.1 & $<0.02$ & 8.8 & 0.02 \\
\hline NO11 & 0.5 & 3500 & 119 & 0.02 & 0.08 & 0.03 & 7.1 & 0.08 \\
\hline $\mathrm{NO} 11 \mathrm{C}$ & 0.4 & 8100 & 113 & 0.01 & 0.07 & 0.02 & 7.9 & 0.06 \\
\hline
\end{tabular}




\begin{tabular}{|c|c|c|c|c|c|c|c|c|}
\hline $\begin{array}{l}\text { Table } 5 \mathrm{~b} . \\
\text { Recalculated to } \mathrm{mg} \\
\text { leached/kg solid }\end{array}$ & & & & & & & & \\
\hline $\mathrm{NA}=$ not analyzed & & & & & & & & \\
\hline Field No. & $\begin{array}{l}\mathrm{Tm} \\
\text { solid }\end{array}$ & $\begin{array}{c}\mathbf{U} \\
\text { solid }\end{array}$ & $\begin{array}{c}\mathbf{V} \\
\text { solid }\end{array}$ & $\begin{array}{c}\text { W } \\
\text { solid }\end{array}$ & $\begin{array}{c}\mathbf{Y} \\
\text { solid }\end{array}$ & $\begin{array}{c}\text { Yb } \\
\text { solid }\end{array}$ & $\begin{array}{c}\text { Zn } \\
\text { solid }\end{array}$ & $\begin{array}{c}\mathrm{Zr} \\
\text { solid }\end{array}$ \\
\hline blank & $<0.0005$ & $<0.01$ & 1.7 & 0.2 & $<0.001$ & $<0.0005$ & 2.4 & $<0.02$ \\
\hline Blank dup & $<0.0005$ & $<0.01$ & 1.6 & 0.2 & 0.002 & 0.0005 & 3.2 & $<0.02$ \\
\hline Chalmette 1 & 0.02 & 0.1 & 7.9 & 0.2 & 1.9 & 0.08 & 73 & 0.1 \\
\hline Chalmette 1 dup & 0.02 & 0.1 & 8.8 & 0.2 & 2.0 & 0.09 & 80 & 0.1 \\
\hline Chalmette 2 & 0.06 & 0.5 & 8.7 & 0.1 & 5.1 & 0.3 & 256 & 0.2 \\
\hline Chalmette 2 dup & 0.05 & 0.5 & 8.2 & 0.1 & 4.7 & 0.29 & 230 & 0.2 \\
\hline NO 7C & 0.01 & 0.1 & 2.8 & 0.1 & 0.8 & 0.06 & 298 & 0.06 \\
\hline NO 7C dup & 0.02 & 0.1 & 3.5 & 0.1 & 1.4 & 0.08 & 469 & 0.08 \\
\hline Blank & $<0.0005$ & $<0.01$ & 1.6 & $<0.05$ & $<0.001$ & $<0.0005$ & 1.8 & $<0.02$ \\
\hline NO1 & 0.03 & 0.4 & 11 & 0.3 & 2.8 & 0.2 & 3980 & 0.8 \\
\hline NO2 & 0.02 & 0.3 & 4.0 & 2.9 & 1.8 & 0.1 & 522 & 0.3 \\
\hline NO2 dup & 0.02 & 0.2 & 4.3 & 2.8 & 1.8 & 0.1 & 520 & 0.3 \\
\hline NO3 & 0.03 & 0.3 & 8.6 & 1.2 & 2.6 & 0.2 & 3410 & 0.6 \\
\hline NO4 & 0.04 & 0.3 & 7.3 & 0.5 & 3.7 & 0.2 & 2500 & 0.6 \\
\hline NO5 & 0.02 & 0.2 & 11 & 0.5 & 2.0 & 0.1 & 7340 & 1.1 \\
\hline NO6 & 0.04 & 0.3 & 6.3 & 3.3 & 3.0 & 0.2 & 729 & 0.4 \\
\hline NO7 A & 0.05 & 0.3 & 14 & 0.9 & 4.1 & 0.3 & 1410 & 0.4 \\
\hline NO7 BC & 0.02 & 0.3 & 5.4 & 3.7 & 1.8 & 0.1 & 721 & 0.4 \\
\hline NO8 BC & 0.03 & 0.3 & 5.0 & 4.8 & 2.2 & 0.2 & 401 & 0.2 \\
\hline NO9 & 0.03 & 0.4 & 7.3 & 5.4 & 2.6 & 0.2 & 324 & 0.4 \\
\hline NO10 & 0.04 & 0.3 & 5.6 & 2.6 & 3.5 & 0.3 & 155 & 0.2 \\
\hline NO11 & 0.04 & 0.3 & 16 & 0.4 & 3.3 & 0.2 & 8450 & 0.7 \\
\hline NO11 C & 0.03 & 0.2 & 13 & 0.5 & 2.8 & 0.2 & 8470 & 0.5 \\
\hline
\end{tabular}




\begin{tabular}{|c|c|}
\hline $\begin{array}{l}\text { Table } 5 \mathrm{~b} . \\
\text { Recalculated to } \mathrm{mg} \\
\text { leached/kg solid }\end{array}$ & \multirow[b]{3}{*}{$\begin{array}{c}\mathrm{Hg} \\
\mathrm{ng} / \mathrm{L}\end{array}$} \\
\hline $\mathrm{NA}=$ not analyzed & \\
\hline Field No. & \\
\hline blank & NA \\
\hline Blank dup & NA \\
\hline Chalmette 1 & NA \\
\hline Chalmette 1 dup & NA \\
\hline Chalmette 2 & NA \\
\hline Chalmette 2 dup & NA \\
\hline NO 7C & NA \\
\hline NO 7C dup & NA \\
\hline Blank & NA \\
\hline $\mathrm{NO1}$ & NA \\
\hline NO2 & NA \\
\hline NO2 dup & NA \\
\hline NO3 & $<15$ \\
\hline $\mathrm{NO} 4$ & NA \\
\hline NO5 & $<15$ \\
\hline NO6 & NA \\
\hline NO7 A & 23 \\
\hline NO7 BC & $<15$ \\
\hline NO8 BC & 18 \\
\hline NO9 & NA \\
\hline NO10 & NA \\
\hline NO11 & $<15$ \\
\hline NO11 C & NA \\
\hline
\end{tabular}


Table 6.

Analytical results for chemical leach tests performed on wet Chalmette samples. Table 6a reports concentrations in the leachate, table $6 \mathrm{~b}$ reports values recalculated to $\mathrm{mg}$ leached per $\mathrm{kg}$ solid. The samples originally contained approximately $60-$ $70 \%$ water, so that measured concentrations would increase proportionally if recalculated to dry weight. All leach tests were run at 1 part solid to 20 parts leach solution. "Modified TCLP" is a USGS modification on EPA Method 1311, using simulated landfill leachate compositions, rotated end over end for 18 hours. Simulated brackish water is a dilute sea-water leach using 6 ppt sea salt in deionized water as the leachate, rotated end over end for 18 hours. "Modified SPLP" is a USGS modification of EPA Method 1312, synthetic rainfall leach test, rotated end over end for 18 hours. "USGS FLT" is a field leach test using deionized water as the leachate, rotated end over end for 5 minutes. "TCLP 1311" method was run by EPA National Enforcement Investigations Center (NEIC) laboratory, using EPA protocols. Concentrations below detection limit shown by $(<)$.

6a. Blank, leachate concentrations

$\mathrm{NA}=$ not analyzed Field ID

\section{Analysis Description}

pH Spec. conduct. $\mu \mathrm{S} / \mathrm{cm}$

\section{Alkalinity CaCO3}

IC-Aq Cl

Blank USGS TCLP Chalmette 1-USGS Chalmette1D-USGS Chalmette 2-USGS Chalmette2D-USGS BlkUSGS SimBrac Chalm 1-USGS Chalm 1D-USGS Chalm 2-USGS Chalm 2D-USGS Blank USGS SPLP Chalme 1-USGS Chalme 1D-USGS Chalmette 2USGS Chalm 2DUSGS Blank USGS FLT Chalm 1USGS

Chalm1D-USGS

Chalm2-USGS

Chalm2D-USGS Blank NEIC-TCLP

Chalmette1-NEIC Chalmette1D-NEIC Chalmette2-NEIC Chalmette2D-NEIC

Ref. Standard

Ref. Standard2

\begin{tabular}{|c|c|c|c|c|c|c|c|}
\hline & & & ppm & ppm & ppm & ppm & ppm \\
\hline Modified TCLP & 4.9 & 4070 & $\mathrm{NA}$ & $\mathrm{NA}$ & $\mathrm{NA}$ & $\mathrm{NA}$ & NA \\
\hline Modified TCLP & 5.0 & 4910 & $\mathrm{NA}$ & $\mathrm{NA}$ & NA & $\mathrm{NA}$ & NA \\
\hline Modified TCLP & 5.1 & 4930 & NA & $\mathrm{NA}$ & $\mathrm{NA}$ & $\mathrm{NA}$ & NA \\
\hline Modified TCLP & 5.2 & 5280 & $\mathrm{NA}$ & $\mathrm{NA}$ & $\mathrm{NA}$ & $\mathrm{NA}$ & NA \\
\hline Modified TCLP & 5.1 & 5220 & NA & $\mathrm{NA}$ & $\mathrm{NA}$ & $\mathrm{NA}$ & NA \\
\hline Simulated brackish water & 8.8 & 8510 & NA & $\mathrm{NA}$ & $\mathrm{NA}$ & $\mathrm{NA}$ & NA \\
\hline Simulated brackish water & 7.0 & 8770 & NA & $\mathrm{NA}$ & $\mathrm{NA}$ & $\mathrm{NA}$ & NA \\
\hline Simulated brackish water & 7.0 & 8770 & NA & $\mathrm{NA}$ & $\mathrm{NA}$ & $\mathrm{NA}$ & NA \\
\hline Simulated brackish water & 7.7 & 9050 & NA & NA & NA & $\mathrm{NA}$ & NA \\
\hline Simulated brackish water & 7.6 & 8900 & $\mathrm{NA}$ & $\mathrm{NA}$ & $\mathrm{NA}$ & $\mathrm{NA}$ & NA \\
\hline Modified SPLP & 4.2 & 29.8 & 1.387 & 1.2 & $<0.08$ & 1.3 & 9.1 \\
\hline Modified SPLP & 7.3 & 1040 & 19.18 & 281 & $<0.08$ & 3.1 & 63 \\
\hline Modified SPLP & 7.2 & 1000 & 19.9 & 288 & $<0.08$ & 3 & 63 \\
\hline Modified SPLP & 8.5 & 1060 & 40.34 & 329 & $<0.08$ & 1.7 & 62 \\
\hline Modified SPLP & 8.5 & 1020 & 39.98 & 281 & $<0.08$ & 1.6 & 56 \\
\hline USGS FLT & 5.8 & 0.65 & 3.988 & $<0.08$ & $<0.08$ & $<0.08$ & $<1.6$ \\
\hline USGS FLT & 7.4 & 643 & 8.516 & 89 & $<0.08$ & 0.8 & 9.1 \\
\hline USGS FLT & 7.4 & 509 & 11.42 & 47 & $<0.08$ & 0.2 & 5.5 \\
\hline USGS FLT & 7.4 & 779 & 8.156 & 25.4 & $<0.08$ & 0.2 & 4.5 \\
\hline USGS FLT & 8.8 & 1000 & 26.76 & 103 & $<0.08$ & 0.4 & 6.7 \\
\hline TCLP 1311 & $\mathrm{NA}$ & $\mathrm{NA}$ & $\mathrm{NA}$ & $\mathrm{NA}$ & $\mathrm{NA}$ & $\mathrm{NA}$ & NA \\
\hline TCLP 1311 & $\mathrm{NA}$ & $\mathrm{NA}$ & NA & $\mathrm{NA}$ & $\mathrm{NA}$ & $\mathrm{NA}$ & NA \\
\hline TCLP 1311 duplicate & $\mathrm{NA}$ & $\mathrm{NA}$ & $\mathrm{NA}$ & $\mathrm{NA}$ & $\mathrm{NA}$ & $\mathrm{NA}$ & NA \\
\hline TCLP 1311 & $\mathrm{NA}$ & $\mathrm{NA}$ & $\mathrm{NA}$ & $\mathrm{NA}$ & $\mathrm{NA}$ & $\mathrm{NA}$ & NA \\
\hline TCLP 1311 duplicate & $\mathrm{NA}$ & $\mathrm{NA}$ & $\mathrm{NA}$ & $\mathrm{NA}$ & $\mathrm{NA}$ & $\mathrm{NA}$ & NA \\
\hline Standard T-103 & $\mathrm{NA}$ & $\mathrm{NA}$ & $\mathrm{NA}$ & $\mathrm{NA}$ & $\mathrm{NA}$ & $\mathrm{NA}$ & NA \\
\hline Standard M-112 & $\mathrm{NA}$ & $\mathrm{NA}$ & NA & $\mathrm{NA}$ & NA & $\mathrm{NA}$ & NA \\
\hline
\end{tabular}


Table 6.

6a. Blank, leachate concentrations

$\mathrm{NA}=$ not analyzed Field ID

Analysis Description

\section{ICPMS LEACH}

\section{ICPMS LEACH}

\section{ICPMS LEACH}

ICPMS LEACH

ICPMS_LEACH

ICPMS_LEACH ICPMS_LEACH

$\mathrm{SO} 4$
$\mathrm{mg} / \mathrm{L}$

$\mathrm{ug} / \mathrm{L}$

Al

As

$\mathrm{Ba}$

Bi

Blank USGS TCLP

Chalmette 1-USGS

Chalmette1D-USGS

Chalmette 2-USGS

Chalmette2D-USGS

BlkUSGS SimBrac

Chalm 1-USGS

Chalm 1D-USGS

Chalm 2-USGS

Chalm 2D-USGS

Blank USGS SPLP

Chalme 1-USGS

Chalme 1D-USGS

Chalmette 2USGS

Chalm 2DUSGS

Blank USGS FLT

Chalm 1USGS

Chalm1D-USGS

Chalm2-USGS

Chalm2D-USGS

Blank NEIC-TCLP

Chalmette1-NEIC

Chalmette1D-NEIC

Chalmette2-NEIC

Chalmette2D-NEIC

Ref. Standard

Modified TCLP

Modified TCLP

Modified TCLP

Modified TCLP

Modified TCLP

Simulated brackish water

Simulated brackish water

Simulated brackish water

Simulated brackish water

Simulated brackish water

Modified SPLP

Modified SPLP

Modified SPLP

Modified SPLP

Modified SPLP

USGS FLT

USGS FLT

USGS FLT

USGS FLT

USGS FLT

TCLP 1311

TCLP 1311

TCLP 1311 duplicate

TCLP 1311

Ref. Standard2

TCLP 1311 duplicate

Standard T-103

Standard M-112

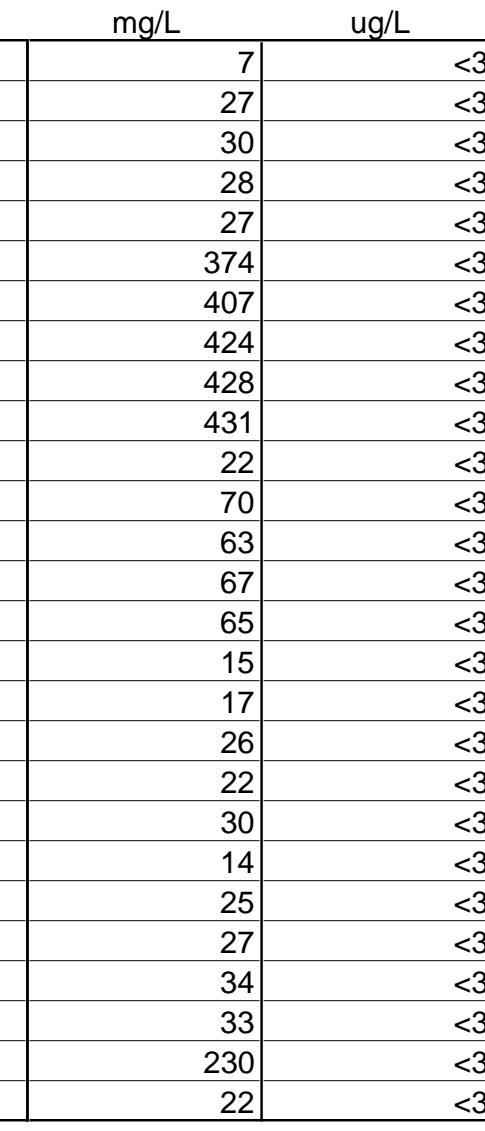

\begin{tabular}{|c|c|c|}
\hline \multirow{2}{*}{\multicolumn{3}{|c|}{$\begin{array}{c}7 . \\
8\end{array}$}} \\
\hline & & \\
\hline & 6 & \\
\hline & 5 & \\
\hline & 5 & \\
\hline & 3. & \\
\hline & 2 & \\
\hline & 5 & \\
\hline & 2 & \\
\hline & 1 & \\
\hline & 8. & \\
\hline & 18 & \\
\hline & 19 & \\
\hline & 20 & \\
\hline & 26 & \\
\hline & 2. & \\
\hline & 3 & 3 \\
\hline & 6 & \\
\hline & 5 & 5 \\
\hline & 21 & \\
\hline & 1 & 1 \\
\hline & 15 & \\
\hline & 11 & \\
\hline & 19 & \\
\hline & 9 & \\
\hline & 12 & \\
\hline & 7. & \\
\hline
\end{tabular}

\begin{tabular}{|c|c|c|c|c|}
\hline & $\mathrm{ug} / \mathrm{L}$ & $\mathrm{ug} / \mathrm{L}$ & $\mathrm{ug} / \mathrm{L}$ & $\mathrm{ug} / \mathrm{L}$ \\
\hline 7.3 & 3.7 & 238 & 0.1 & 0.2 \\
\hline 82 & 16 & 358 & 0.2 & $<0.2$ \\
\hline 66 & 14 & 281 & 0.07 & $<0.2$ \\
\hline 55 & 33 & 387 & 0.4 & $<0.2$ \\
\hline 59 & 29 & 378 & 0.5 & $<0.2$ \\
\hline 3.4 & 7.9 & 106 & $<0.05$ & $<0.2$ \\
\hline 21 & 11 & 192 & $<0.05$ & $<0.2$ \\
\hline 59 & 9.5 & 188 & $<0.05$ & $<0.2$ \\
\hline 20 & 11 & 159 & $<0.05$ & $<0.2$ \\
\hline 11 & 10 & 155 & $<0.05$ & $<0.2$ \\
\hline 8.2 & $<1$ & 2.7 & $<0.05$ & $<0.2$ \\
\hline 186 & $<1$ & 151 & $<0.05$ & $<0.2$ \\
\hline 192 & 2 & 16 & $<0.05$ & $<0.2$ \\
\hline 208 & 2 & 53 & $<0.05$ & $<0.2$ \\
\hline 67 & 1 & 27 & $<0.05$ & $<0.2$ \\
\hline 2.9 & $<1$ & 18 & $<0.05$ & $<0.2$ \\
\hline 38 & $<1$ & 160 & $<0.05$ & $<0.2$ \\
\hline 67 & $<1$ & 189 & $<0.05$ & $<0.2$ \\
\hline 50 & $<1$ & 185 & $<0.05$ & $<0.2$ \\
\hline 211 & 2 & 19 & $<0.05$ & $<0.2$ \\
\hline 16 & $<1$ & 97 & $<0.05$ & $<0.2$ \\
\hline 152 & 18 & 258 & 0.06 & $<0.2$ \\
\hline 114 & 20 & 267 & 0.2 & $<0.2$ \\
\hline 196 & 35 & 404 & 0.9 & $<0.2$ \\
\hline 91 & 35 & 438 & 0.8 & $<0.2$ \\
\hline 128 & 3.8 & 42 & 4.9 & $<0.2$ \\
\hline .6 & $<1$ & 3 & $<0.05$ & $<0.2$ \\
\hline
\end{tabular}


Table 6.

6a. Blank, leachate concentrations

$\mathrm{NA}=$ not analyzed Field ID

Analysis Description

\section{ICPMS LEACH}

\section{ICPMS LEACH}

\section{ICPMS_LEACH}

\section{ICPMS LEACH}

\section{ICPMS LEACH}

\section{ICPMS_LEACH ICPMS_LEACH}

$\mathrm{mg} / \mathrm{L}$

$\mathrm{Cd}$
$\mathrm{ug} / \mathrm{L}$

$\mathrm{ug} / \mathrm{L}$

Co

$\mathrm{ug} / \mathrm{L}$

Cs

$\mathrm{Cu}$

Blank USGS TCLP

Chalmette 1-USGS

Chalmette1D-USGS

Chalmette 2-USGS

Chalmette2D-USGS

BlkUSGS SimBrac

Chalm 1-USGS

Chalm 1D-USGS

Chalm 2-USGS

Chalm 2D-USGS

Blank USGS SPLP

Chalme 1-USGS

Chalme 1D-USGS

Chalmette 2USGS

Chalm 2DUSGS

Blank USGS FLT

Chalm 1USGS

Chalm1D-USGS

Chalm2-USGS

Chalm2D-USGS

Blank NEIC-TCLP

Chalmette1-NEIC

Chalmette1D-NEIC

Chalmette2-NEIC

Chalmette2D-NEIC

Ref. Standard

Modified TCLP

Modified TCLP

Modified TCLP

Modified TCLP

Modified TCLP

Simulated brackish water

Simulated brackish water

Simulated brackish water

Simulated brackish water

Simulated brackish water

Modified SPLP

Modified SPLP

Modified SPLP

Modified SPLP

Modified SPLP

USGS FLT

USGS FLT

USGS FLT

USGS FLT

USGS FLT

TCLP 1311

TCLP 1311

TCLP 1311 duplicate

TCLP 1311

Ref. Standard2

TCLP 1311 duplicate

Standard T-103

Standard M-112

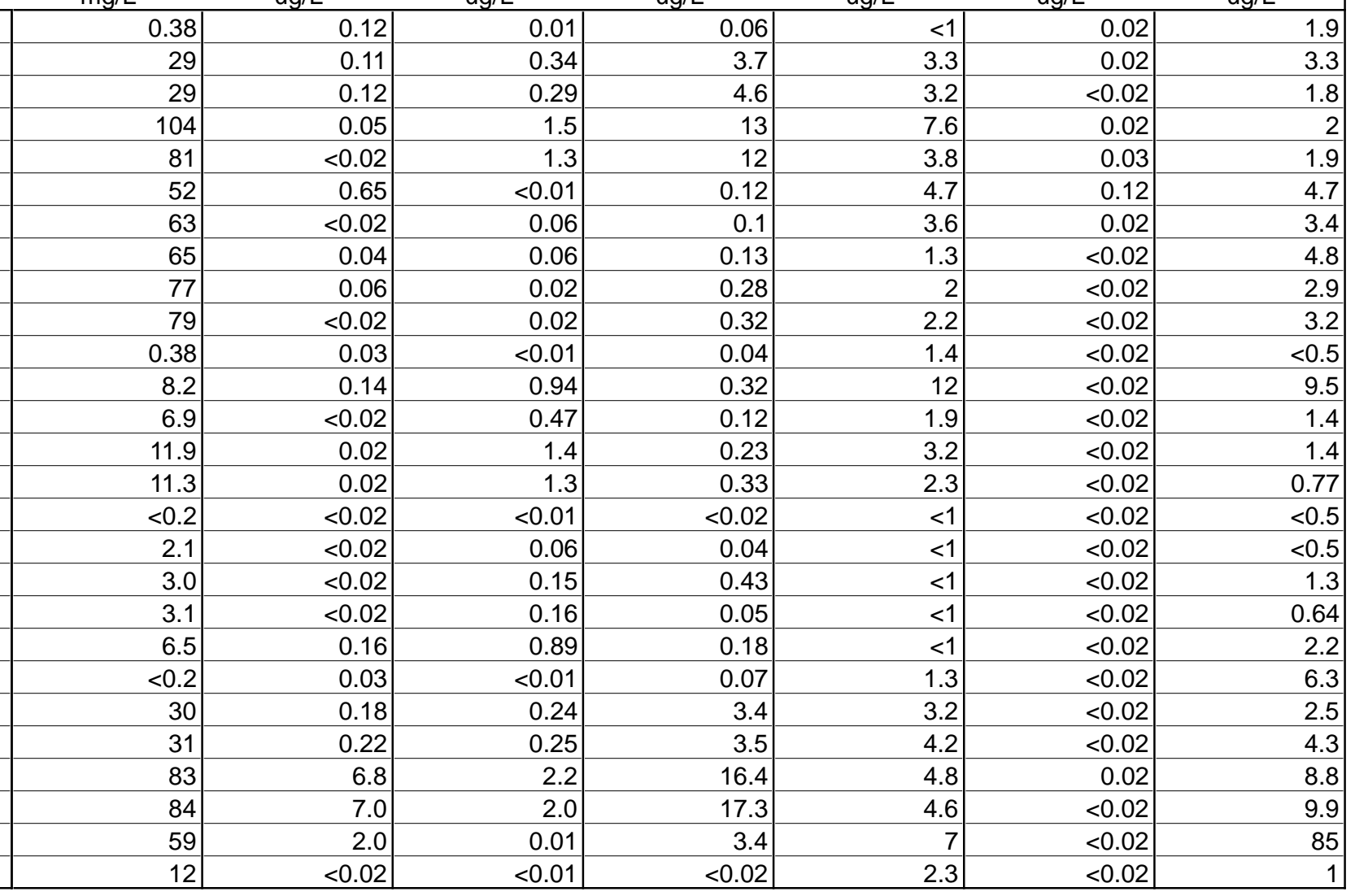

$\mathrm{ug} / \mathrm{L}$ 
Table 6.

6a. Blank, leachate concentrations

$\mathrm{NA}=$ not analyzed Field ID

\section{Analysis Description}

\section{ICPMS LEACH}

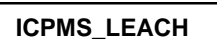

\section{ICPMS LEACH}

\section{ICPMS LEACH}

ICPMS_LEACH

ICPMS_LEACH ICPMS_LEACH

Blank USGS TCLP

Chalmette 1-USGS

Chalmette1D-USGS

Chalmette 2-USGS

Chalmette2D-USGS

BlkUSGS SimBrac

Chalm 1-USGS

Chalm 1D-USGS

Chalm 2-USGS

Chalm 2D-USGS

Blank USGS SPLP

Chalme 1-USGS

Chalme 1D-USGS

Chalmette 2USGS

Chalm 2DUSGS

Blank USGS FLT

Chalm 1USGS

Chalm1D-USGS

Chalm2-USGS

Chalm2D-USGS

Blank NEIC-TCLP

Chalmette1-NEIC

Chalmette1D-NEIC

Chalmette2-NEIC

Chalmette2D-NEIC

Ref. Standard

Modified TCLP

Modified TCLP

Modified TCLP

Modified TCLP

Modified TCLP

Simulated brackish water

Simulated brackish water

Simulated brackish water

Simulated brackish water

Simulated brackish water

Modified SPLP

Modified SPLP

Modified SPLP

Modified SPLP

Modified SPLP

USGS FLT

USGS FLT

USGS FLT

USGS FLT

USGS FLT

TCLP 1311

TCLP 1311

TCLP 1311 duplicate

TCLP 1311

Ref. Standard2

TCLP 1311 duplicate

Standard T-103

Standard M-112

\begin{tabular}{|c|c|c|c|c|c|c|}
\hline $\begin{array}{c}\text { Dy } \\
\text { ug/L }\end{array}$ & $\begin{array}{c}\mathrm{Er} \\
\mathrm{ug} / \mathrm{L}\end{array}$ & $\begin{array}{c}\mathrm{Eu} \\
\mathrm{ug} / \mathrm{L}\end{array}$ & $\begin{array}{c}\mathrm{Fe} \\
\mathrm{ug} / \mathrm{L}\end{array}$ & $\begin{array}{c}\mathrm{Ga} \\
\mathrm{ug} / \mathrm{L}\end{array}$ & $\begin{array}{c}\text { Gd } \\
\mathrm{ug} / \mathrm{L}\end{array}$ & $\begin{array}{r}\text { Ge } \\
\mathrm{ug} / \mathrm{L}\end{array}$ \\
\hline 0.26 & $<0.005$ & $<0.005$ & $<50$ & $<0.05$ & $<0.005$ & $<0.05$ \\
\hline$<0.005$ & 0.007 & 0.03 & $<50$ & $<0.05$ & 0.06 & $<0.05$ \\
\hline 0.03 & 0.02 & 0.01 & $<50$ & 0.06 & 0.04 & 0.06 \\
\hline 0.16 & 0.10 & 0.10 & 27200 & 0.1 & 0.3 & 0.08 \\
\hline 0.18 & 0.10 & 0.09 & 29500 & 0.1 & 0.2 & $<0.05$ \\
\hline$<0.005$ & $<0.005$ & $<0.005$ & $<50$ & $<0.05$ & 0.009 & $<0.05$ \\
\hline$<0.005$ & 0.01 & $<0.005$ & $<50$ & $<0.05$ & 0.006 & $<0.05$ \\
\hline$<0.005$ & 0.007 & $<0.005$ & $<50$ & 0.07 & 0.03 & $<0.05$ \\
\hline$<0.005$ & $<0.005$ & $<0.005$ & $<50$ & $<0.05$ & 0.03 & $<0.05$ \\
\hline$<0.005$ & 0.01 & $<0.005$ & $<50$ & $<0.05$ & 0.01 & $<0.05$ \\
\hline$<0.005$ & $<0.005$ & 0.008 & $<50$ & $<0.05$ & 0.02 & $<0.05$ \\
\hline 0.04 & 0.01 & 0.03 & $<50$ & $<0.05$ & 0.08 & $<0.05$ \\
\hline 0.03 & 0.006 & 0.01 & $<50$ & 0.07 & 0.04 & $<0.05$ \\
\hline 0.1 & 0.19 & 0.02 & $<50$ & 0.09 & 0.2 & $<0.05$ \\
\hline 0.055 & 0.05 & 0.03 & $<50$ & 0.1 & 0.1 & $<0.05$ \\
\hline$<0.005$ & $<0.005$ & 0.006 & $<50$ & $<0.05$ & 0.02 & $<0.05$ \\
\hline$<0.005$ & 0.01 & $<0.005$ & $<50$ & $<0.05$ & 0.01 & $<0.05$ \\
\hline$<0.005$ & $<0.005$ & 0.02 & $<50$ & $<0.05$ & 0.03 & $<0.05$ \\
\hline$<0.005$ & $<0.005$ & 0.009 & $<50$ & $<0.05$ & 0.05 & 0.05 \\
\hline 0.063 & 0.02 & 0.02 & $<50$ & 0.09 & 0.10 & $<0.05$ \\
\hline$<0.005$ & $<0.005$ & $<0.005$ & $<50$ & $<0.05$ & $<0.005$ & $<0.05$ \\
\hline 0.01 & 0.01 & 0.009 & $<50$ & $<0.05$ & 0.04 & $<0.05$ \\
\hline 0.006 & 0.01 & 0.02 & $<50$ & $<0.05$ & 0.07 & 0.07 \\
\hline 0.28 & 0.13 & 0.08 & $<50$ & 0.2 & 0.5 & 0.2 \\
\hline 0.2 & 0.19 & 0.08 & $<50$ & 0.1 & 0.6 & 0.2 \\
\hline$<0.005$ & $<0.005$ & $<0.005$ & $<50$ & 0.05 & 0.005 & $<0.05$ \\
\hline$<0.005$ & $<0.005$ & $<0.005$ & $<50$ & $<0.05$ & $<0.005$ & $<0.05$ \\
\hline
\end{tabular}


Table 6.

6a. Blank, leachate concentrations

$\mathrm{NA}=$ not analyzed Field ID

\section{Analysis Description}

Blank USGS TCLP Chalmette 1-USGS

Chalmette1D-USGS

Chalmette 2-USGS

Chalmette2D-USGS

BlkUSGS SimBrac

Chalm 1-USGS

Chalm 1D-USGS

Chalm 2-USGS

Chalm 2D-USGS

Blank USGS SPLP

Chalme 1-USGS

Chalme 1D-USGS

Chalmette 2USGS

Chalm 2DUSGS

Blank USGS FLT

Chalm 1USGS

Chalm1D-USGS

Chalm2-USGS

Chalm2D-USGS

Blank NEIC-TCLP

Chalmette1-NEIC

Chalmette1D-NEIC

Chalmette2-NEIC

Chalmette2D-NEIC

Ref. Standard

Ref. Standard2

CVAFS

ICPMS_LEACH

\section{ICPMS LEACH}

ICPMS LEACH

ICPMS_LEACH

ICPMS_LEACH ICPMS_LEACH

Modified TCL

Modified TCLP

Modified TCLP

Modified TCLP

Modified TCLP

Simulated brackish water

Simulated brackish water

Simulated brackish water

Simulated brackish water

Simulated brackish water

Modified SPLP

Modified SPLP

Modified SPLP

Modified SPLP

Modified SPLP

USGS FLT

USGS FLT

USGS FLT

USGS FLT

USGS FLT

TCLP 1311

TCLP 1311

TCLP 1311 duplicate

TCLP 1311

TCLP 1311 duplicate

Standard T-103

Standard M-112

ng/L $\quad$ ug/L

$\mathrm{mg} / \mathrm{L}$

$\mathrm{La}$
$\mathrm{ug} / \mathrm{L}$

$\mathrm{Li}$
$\mathrm{ug} / \mathrm{L}$

Lu

$\mathrm{Mg}$

mg/L $\quad \mathrm{ug} / \mathrm{L}$

\begin{tabular}{r|r|r|r} 
& $\mathrm{ug} / \mathrm{L}$ & $\mathrm{ug} / \mathrm{L}$ \\
\hline
\end{tabular}

$<0.1$

$\mathrm{mg} / \mathrm{L}$

\begin{tabular}{l|r|r|r|r|}
$<15$ & 0.01 & 2.3 & 0.01 & 24 \\
\hline$<15$ & 0.005 & 15.5 & 0.17 & 19 \\
\hline
\end{tabular}

\begin{tabular}{r|r}
$<15$ & 0.005 \\
\hline
\end{tabular}

$<15$

0.05

0.03

\begin{tabular}{l|l}
$<15$ & $<0.005$ \\
$<15$ & $<0.005$
\end{tabular}

$<15$

$<0.005$

$<0.005$

$<15$

$<15$

$<0.005$

$<15$

$<15$

$<15$

$<15$

$<15$

$<15$

$<15$

$<15$

$<15$

$<15$

$<15$

$<15$

$<15$

$<0.005$

$\begin{array}{r}0.01 \\ 0.008 \\ \hline\end{array}$

0.02

0.03
$<0.005$

$<0.005$

$<0.005$

$<0.005$

0.01
$<0.005$

$<0.005$

0.007

0.05

0.07

$<0.005$

$<0.005$

15
25
24

5

0.76

0.76

\begin{tabular}{r|r}
0.64 & 15 \\
\hline 0.01
\end{tabular}

\begin{tabular}{|r|r|}
$<<0.1$ & 0.06 \\
\hline$<0.1$ & 39 \\
\hline$<0.1$ & 38 \\
\hline$<0.1$ & 54 \\
\hline$<0.1$ & 52 \\
\hline 00.1 & 152 \\
\hline
\end{tabular}

\begin{tabular}{|c|c|c|c|c|}
\hline 55 & 0.01 & 1490 & $<0.1$ & 152 \\
\hline 60 & 0.04 & 1340 & $<0.1$ & 168 \\
\hline
\end{tabular}

\begin{tabular}{r|r|r|}
62 & 0.04 & 1340 \\
\hline 65 & 0.03 & 1300 \\
\hline
\end{tabular}

\begin{tabular}{l|l|r|}
65 & 0.02 & 1260 \\
\hline 66 & 0.01 & 1300 \\
\hline
\end{tabular}

\begin{tabular}{r|r|r|}
66 & 0.01 & 1300 \\
\hline 0.2 & 0.02 & 9.9 \\
\hline
\end{tabular}

\begin{tabular}{r|r|r|}
\hline 10 & 0.44 & $<0.9$ \\
\hline 9 & 0.23 & $<0.9$ \\
\hline
\end{tabular}

\begin{tabular}{r|r|r|}
9 & 0.23 & $<0.9$ \\
15 & 0.7 & $<0.9$ \\
\hline
\end{tabular}

\begin{tabular}{r|r|r|}
14 & 0.66 & $<0.9$ \\
\hline 0.07 & $<0.01$ & $<0.9$ \\
\hline
\end{tabular}

\begin{tabular}{rr|r|}
\hline 2.4 & $<.04$ & $<0.9$ \\
\hline & 0.09 & $<0.9$ \\
\hline
\end{tabular}

\begin{tabular}{r|r|r|}
2.4 & 0.04 & $<0.9$ \\
\hline 4.2 & 0.09 & $<0.9$ \\
\hline 2.7 & 0.1 & $<0.9$ \\
\hline
\end{tabular}

\begin{tabular}{r|r|r|r|r|}
\hline 11 & 0.54 & $<$ & $<0.1$ & 9.6 \\
\hline 0.4 & 0.01 & 8.9 & $<0.1$ & 0.03 \\
\hline 14 & 0.13 & 7 & $<0.1$ & 39 \\
\hline 24 & 0.14 & 12 & $<0.1$ & 41 \\
\hline 24 & 1.1 & 5.4 & $<0.1$ & 53 \\
\hline 3.6 & 1.0 & 12 & $<0.1$ & 54 \\
\hline 3.3 & 0.02 & 29 & $<0.1$ & 34 \\
\hline
\end{tabular}


Table 6.

6a. Blank, leachate concentrations

$\mathrm{NA}=$ not analyzed Field ID

Analysis Description

\title{
ICPMS LEACH
}

\section{ICPMS LEACH}

\section{ICPMS LEACH}

\section{ICPMS LEACH}

\section{ICPMS LEACH}

$\mathrm{Na}$
$\mathrm{mg} / \mathrm{L}$

$\mathrm{Nb}$
$\mathrm{ug} / \mathrm{L}$

Nd

ICPMS_LEACH ICPMS_LEACH

Blank USGS TCLP

Chalmette 1-USGS

Chalmette1D-USGS

Chalmette 2-USGS

Chalmette2D-USGS

BlkUSGS SimBrac

Chalm 1-USGS

Chalm 1D-USGS

Chalm 2-USGS

Chalm 2D-USGS

Blank USGS SPLP

Chalme 1-USGS

Chalme 1D-USGS

Chalmette 2USGS

Chalm 2DUSGS

Blank USGS FLT

Chalm 1USGS

Chalm1D-USGS

Chalm2-USGS

Chalm2D-USGS

Blank NEIC-TCLP

Chalmette1-NEIC

Chalmette1D-NEIC

Chalmette2-NEIC

Chalmette2D-NEIC

Ref. Standard

Modified TCLP

Modified TCLP

Modified TCLP

Modified TCLP

Modified TCLP

Simulated brackish water

Simulated brackish water

Simulated brackish water

Simulated brackish water

Simulated brackish water

Modified SPLP

Modified SPLP

Modified SPLP

Modified SPLP

Modified SPLP

USGS FLT

USGS FLT

USGS FLT

USGS FLT

USGS FLT

TCLP 1311

TCLP 1311

TCLP 1311 duplicate

TCLP 1311

Ref. Standard2

TCLP 1311 duplicate

Standard T-103

Standard M-112

\begin{tabular}{|c|c|}
\hline$<0.2$ & 6.3 \\
\hline 1370 & 3 \\
\hline 1300 & $<2$ \\
\hline 7500 & $<2$ \\
\hline 6950 & $<2$ \\
\hline 9.1 & $<2$ \\
\hline 3.9 & 6.2 \\
\hline 4 & 11 \\
\hline 1780 & 8.7 \\
\hline 1840 & 7.4 \\
\hline 5.3 & $<2$ \\
\hline 15 & $g$ \\
\hline 13 & 5.4 \\
\hline 207 & 7.2 \\
\hline 179 & 7.2 \\
\hline$<0.2$ & $<2$ \\
\hline 1.2 & $<2$ \\
\hline 0.2 & $<2$ \\
\hline 11 & $<2$ \\
\hline 41 & $<2$ \\
\hline$<0.2$ & $<2$ \\
\hline 1430 & $<2$ \\
\hline 1510 & $<2$ \\
\hline 7110 & $<2$ \\
\hline 7260 & $<2$ \\
\hline 7.2 & 32 \\
\hline 0.3 & 4.8 \\
\hline
\end{tabular}

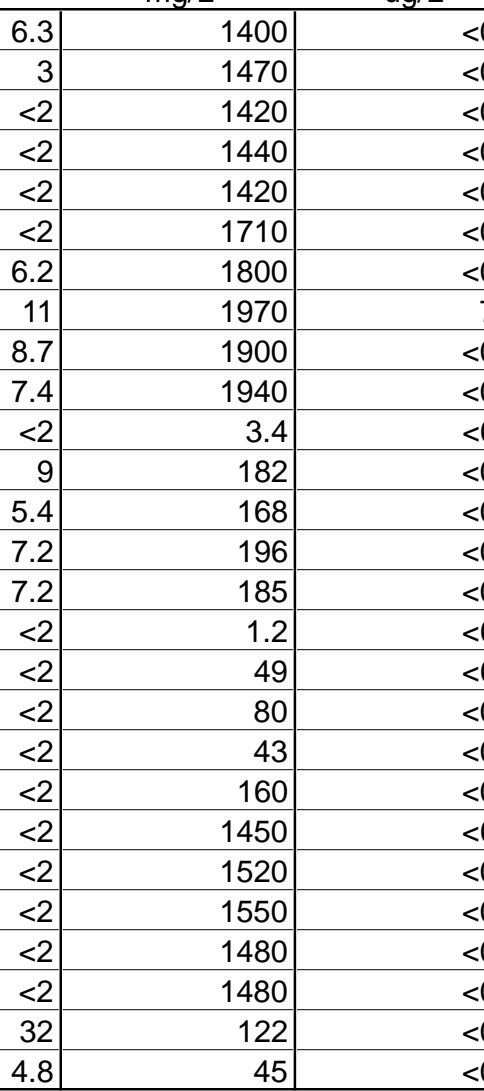

$<0.2$
$<0.2$

\begin{tabular}{l|l|}
$<0.2$ & 0.02 \\
\hline
\end{tabular}

$<0.2 \quad 0.2$

$<0.2$

$<0.2 \quad 0.99$

\begin{tabular}{l|l|}
$<0.2$ & 0.83 \\
$<0.2$ & 0.02 \\
\hline$<0.2$ & 0.02 \\
\hline
\end{tabular}

\begin{tabular}{r|}
7.4 \\
\hline
\end{tabular}$\quad 0.02$

\begin{tabular}{r|r|}
7.4 & 0.06 \\
$<0.2$ & 0.03 \\
\hline
\end{tabular}

$<0.2$

$<0.2$

$<0.2 \quad 0.46$

\begin{tabular}{l|l|}
$<0.2$ & 0.22 \\
\hline
\end{tabular}

$<0.2$

$<0.2$

$<0.2$

$<0.2$

$<0.2$

$<0.2$

$<0.2$

$<0.2$

$<0.2$

$<0.2$

$<0.2$

$<0.2$

0.76

0.76
0.58
$<0.01$

$\begin{array}{r}<0.01 \\ 0.03 \\ \hline\end{array}$

$\begin{array}{r}0.03 \\ 0.04 \\ \hline\end{array}$

\begin{tabular}{l}
0.09 \\
0.55 \\
\hline
\end{tabular}

0.55
0.02

0.17

0.22
1.4

$\begin{array}{r}1.4 \\ 1.5 \\ \hline 0.02\end{array}$

2

\begin{tabular}{ll} 
& \\
\hline
\end{tabular}

\begin{abstract}
4.8
\end{abstract}

\begin{tabular}{|r|r|}
\hline & 0.02 \\
\hline
\end{tabular}

\begin{tabular}{|c|c|}
\hline $\begin{array}{c}\text { ICPMS_LEACH } \\
\mathbf{N i} \\
\mathrm{ug} / \mathrm{L}\end{array}$ & $\begin{array}{c}\text { ICPMS_LEACH } \\
\mathbf{P} \\
\mathrm{mg} / \mathrm{L}\end{array}$ \\
\hline 1.4 & $<0.01$ \\
\hline 4.6 & 0.3 \\
\hline 4.8 & 0.3 \\
\hline 33 & 0.1 \\
\hline 32 & 0.2 \\
\hline 3.5 & 0.05 \\
\hline 1.2 & 0.04 \\
\hline 0.8 & 0.1 \\
\hline 2.1 & 0.08 \\
\hline 2.5 & 0.05 \\
\hline 4 & $<0.01$ \\
\hline 9.7 & $<0.01$ \\
\hline 4.6 & $<0.01$ \\
\hline 5.3 & 0.07 \\
\hline 5 & 0.06 \\
\hline$<0.4$ & $<0.01$ \\
\hline$<0.4$ & $<0.01$ \\
\hline$<0.4$ & $<0.01$ \\
\hline$<0.4$ & $<0.01$ \\
\hline 5.7 & $<0.01$ \\
\hline 1.0 & $<0.01$ \\
\hline 6.3 & 0.4 \\
\hline 6.8 & 0.4 \\
\hline 38 & 0.02 \\
\hline 39 & $<0.01$ \\
\hline 9.5 & 0.4 \\
\hline$<0.4$ & 1.2 \\
\hline
\end{tabular}


Table 6.

6a. Blank, leachate concentrations

$\mathrm{NA}=$ not analyzed Field ID

\section{Analysis Description}

\section{ICPMS LEACH}

ICPMS_LEACH

\section{ICPMS LEACH}

\section{ICPMS LEACH}

\section{ICPMS LEACH}

$\begin{array}{cc}\mathrm{Rb} & \mathbf{S b} \\ \mathrm{ug} / \mathrm{L} & \mathrm{ug} / \mathrm{L}\end{array}$

$\mathrm{Sc}$
$\mathrm{ug} / \mathrm{L}$

ICPMS_LEACH ICPMS_LEACH

$\mathrm{ug} / \mathrm{L}$

$\mathrm{ug} / \mathrm{L}$

$<0.3$

ug/L

$\mathrm{SiO} 2$

\begin{tabular}{l} 
Blank USGS TCLP \\
\hline Chalmette 1-USGS
\end{tabular}

Chalmette1D-USGS

Chalmette 2-USGS

Chalmette2D-USGS

BlkUSGS SimBrac

Chalm 1-USGS

Chalm 1D-USGS

Chalm 2-USGS

Chalm 2D-USGS

Blank USGS SPLP

Chalme 1-USGS

Chalme 1D-USGS

Chalmette 2USGS

Chalm 2DUSGS

Blank USGS FLT

Chalm 1USGS

Chalm1D-USGS

Chalm2-USGS

Chalm2D-USGS

Blank NEIC-TCLP

Chalmette1-NEIC

Chalmette1D-NEIC

Chalmette2-NEIC

Chalmette2D-NEIC

Ref. Standard

Modified TCLP

Modified TCLP

Modified TCLP

Modified TCLP

Simulated brackish water

Simulated brackish water

Simulated brackish water

Simulated brackish water

Simulated brackish water

Modified SPLP

Modified SPLP

Modified SPLP

Modified SPLP

Modified SPLP

USGS FLT

USGS FLT

USGS FLT

USGS FLT

USGS FLT

TCLP 1311

TCLP 1311

TCLP 1311 duplicate

TCLP 1311

Ref. Standard2

TCLP 1311 duplicate

Standard T-103

Standard M-112

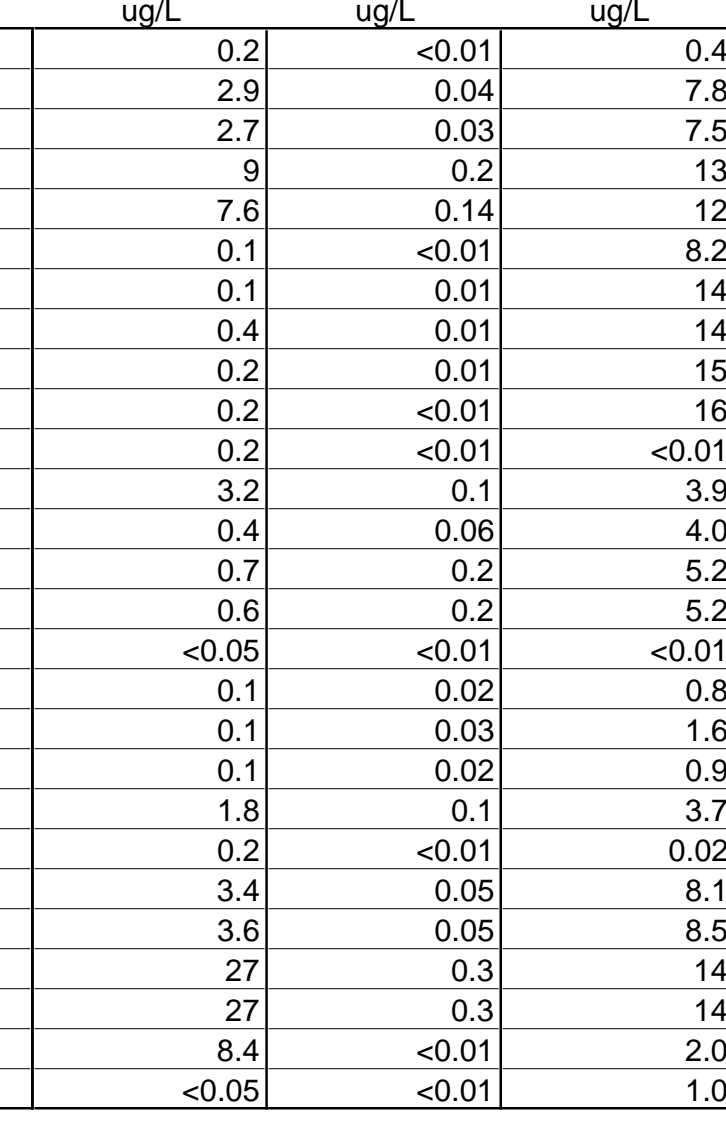

\begin{tabular}{r|r}
\hline 7.4 & $<$ \\
\hline 7.5 & $<0.3$ \\
13 & $<$ \\
12 & $<0.3$ \\
\hline 8.2 & $<0.3$ \\
14 & $<0.3$ \\
14 & $<0.3$ \\
15 & $<0.3$ \\
16 & $<0.3$ \\
\hline 01 & $<0.3$ \\
\hline 3.9 & $<0.3$ \\
4.0 & $<0.3$ \\
\hline 5.2 & $<0.3$ \\
\hline 5.2 & $<0.3$ \\
\hline 01 & $<0.3$ \\
\hline 0.8 & $<0.3$ \\
1.6 & $<0.3$ \\
\hline 0.9 & $<0.3$ \\
\hline 3.7 & $<0.3$ \\
\hline 02 & $<0.3$ \\
\hline 8.1 & $<0.3$ \\
\hline 8.5 & $<0.3$ \\
\hline 14 & $<0.3$ \\
\hline 14 & $<0.3$ \\
\hline 2.0 & $<.1$ \\
\hline 1.0 & $<0.3$ \\
\hline
\end{tabular}

\begin{tabular}{l|l|}
$<0.3$ & $<0.6$ \\
$<0.3$ & $<0.6$ \\
\hline
\end{tabular}

$<0.3$

$<0.3$

$<0.3$

$<0.3$

$<0.3$

$<0.3$

$<0.3$

$<0.3$

$<0.3$

$<0.3$

$<0.3$

$<0.3$

$<0.3$

$<0.3$

$<0.3$

$<0.3$

$<0.3$

$<0.3$

$<0.3$

9.1

$<0.3$

\begin{tabular}{r|r|r|}
\hline$<0.6$ & 5.1 & $<0.2$ \\
\hline$<0.6$ & 6.8 & 5.1 \\
\hline 0.6 & 14 & 4.4 \\
\hline$<0.6$ & 6.9 & 8.8 \\
\hline$<0.6$ & 5.9 & 8.5 \\
\hline$<0.6$ & 52 & $<0.2$ \\
\hline$<0.6$ & 53 & 1.8 \\
\hline$<0.6$ & 54 & 1.5 \\
\hline$<0.6$ & 53 & 3.2 \\
\hline$<0.6$ & 54 & 3.1 \\
\hline$<0.6$ & 4.4 & $<0.2$ \\
\hline$<0.6$ & 7.4 & 1.7 \\
\hline$<0.6$ & 6.5 & 2 \\
\hline$<0.6$ & 9.7 & 3.9 \\
\hline$<0.6$ & 6.4 & 3.6 \\
\hline$<0.6$ & 3.6 & $<0.2$ \\
\hline$<0.6$ & 4.6 & $<0.2$ \\
\hline$<0.6$ & 6.4 & $<0.2$ \\
\hline$<0.6$ & 3.7 & $<0.2$ \\
\hline$<0.6$ & 7.1 & $<0.2$ \\
\hline$<0.6$ & 2.7 & $<0.2$ \\
\hline$<0.6$ & 8.3 & 4.3 \\
\hline$<0.6$ & 5.4 & 4.9 \\
\hline$<0.6$ & 6.7 & 9.9 \\
\hline$<0.6$ & 8.2 & 9.4 \\
\hline 0.8 & 5 & 8 \\
\hline$<0.6$ & $<1$ & 4.1 \\
\hline & &
\end{tabular}


Table 6.

6a. Blank, leachate concentrations

$\mathrm{NA}=$ not analyzed Field ID

\section{Analysis Description}

\section{ICPMS LEACH}

\section{ICPMS LEACH}

\section{ICPMS LEACH}

\section{ICPMS LEACH}

\section{ICPMS LEACH}

Th

ICPMS_LEACH ICPMS_LEACH

Blank USGS TCLP

Chalmette 1-USGS

Chalmette1D-USGS

Chalmette 2-USGS

Chalmette2D-USGS

BlkUSGS SimBrac

Chalm 1-USGS

Chalm 1D-USGS

Chalm 2-USGS

Chalm 2D-USGS

Blank USGS SPLP

Chalme 1-USGS

Chalme 1D-USGS

Chalmette 2USGS

Chalm 2DUSGS

Blank USGS FLT

Chalm 1USGS

Chalm1D-USGS

Chalm2-USGS

Chalm2D-USGS

Blank NEIC-TCLP

Chalmette1-NEIC

Chalmette1D-NEIC

Chalmette2-NEIC

Chalmette2D-NEIC

Ref. Standard

Modified TCLP

$\mathrm{ug} / \mathrm{L}$

$\mathrm{ug} / \mathrm{L}$

$\mathrm{ug} / \mathrm{L}$

$\mathrm{ug} / \mathrm{L}$

$\mathrm{ug} / \mathrm{L}$

TI

\section{Modified TCLP}

Modified TCLP

Modified TCLP

Modified TCLP

Simulated brackish water

Simulated brackish water

Simulated brackish water

Simulated brackish water

Simulated brackish water

Modified SPLP

Modified SPLP

Modified SPLP

Modified SPLP

Modified SPLP

USGS FLT

USGS FLT

USGS FLT

USGS FLT

USGS FLT

TCLP 1311

TCLP 1311

TCLP 1311 duplicate

TCLP 1311

Ref. Standard2

TCLP 1311 duplicate

Standard T-103

Standard M-112

\begin{tabular}{|c|c|c|}
\hline$<0.01$ & 30.5 & $<0.02$ \\
\hline 0.05 & 338 & $<0.02$ \\
\hline$<0.01$ & 333 & $<0.02$ \\
\hline 0.2 & 620 & $<0.02$ \\
\hline 0.2 & 524 & $<0.02$ \\
\hline$<0.01$ & 1600 & $<0.02$ \\
\hline$<0.01$ & 1420 & $<0.02$ \\
\hline 0.09 & 1450 & 4.3 \\
\hline$<0.01$ & 1430 & 0.7 \\
\hline$<0.01$ & 1450 & $<0.02$ \\
\hline$<0.01$ & 3 & $<0.02$ \\
\hline 0.03 & 94 & $<0.02$ \\
\hline 0.02 & 86 & $<0.02$ \\
\hline 0.1 & 102 & $<0.02$ \\
\hline 0.1 & 99 & $<0.02$ \\
\hline$<0.01$ & $<0.5$ & $<0.02$ \\
\hline$<0.01$ & 25 & $<0.02$ \\
\hline 0.02 & 37 & $<0.02$ \\
\hline$<0.01$ & 28 & $<0.02$ \\
\hline 0.06 & 62 & $<0.02$ \\
\hline$<0.01$ & 1.6 & $<0.02$ \\
\hline$<0.01$ & 324 & $<0.02$ \\
\hline$<0.01$ & 343 & $<0.02$ \\
\hline 0.6 & 532 & $<0.02$ \\
\hline 0.3 & 544 & $<0.02$ \\
\hline$<0.01$ & 787 & 0.2 \\
\hline$<0.01$ & 78 & $<0.02$ \\
\hline
\end{tabular}

$<0.00$

\begin{tabular}{l|l}
0.01 & $<0.2$ \\
\hline & $<0.2$
\end{tabular}

$<0.005$

$<.005$
0.04

\begin{tabular}{|r|r|r|}
\hline 2 & 0.04 & $<0.2$ \\
\hline & 0.03 & $<0.2$ \\
\hline
\end{tabular}

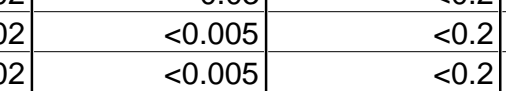

$<0.005$

\begin{tabular}{l}
$<0.005$ \\
\hline 0.005
\end{tabular}

\begin{tabular}{|l|l|}
\hline & $<0.2$ \\
\hline & $<0.005$
\end{tabular}

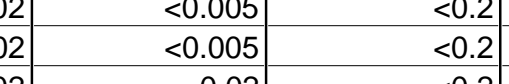

0.02

0.006
0.02

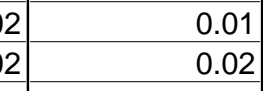

\begin{tabular}{l}
0.005 \\
\hline 0.02
\end{tabular}

$<0.005$

$<0.005$

\begin{tabular}{|r|r|}
\hline 0.02 & $<0.005$ \\
\hline 0.02 & 0.005 \\
\hline
\end{tabular}

\begin{tabular}{|r|r|}
\hline & 0.005 \\
\hline 0.005
\end{tabular}

\begin{tabular}{r|r|}
0.005 \\
\hline
\end{tabular}

\begin{tabular}{|r|r|}
\hline 0.02 & 0.01 \\
\hline 0.02 & 0.05 \\
\hline
\end{tabular}

0.05
0.2

\begin{tabular}{l|l}
0.2 & $<0.005$ \\
\hline 0.02 & $<0.005$
\end{tabular}

$<0.005<0.2$

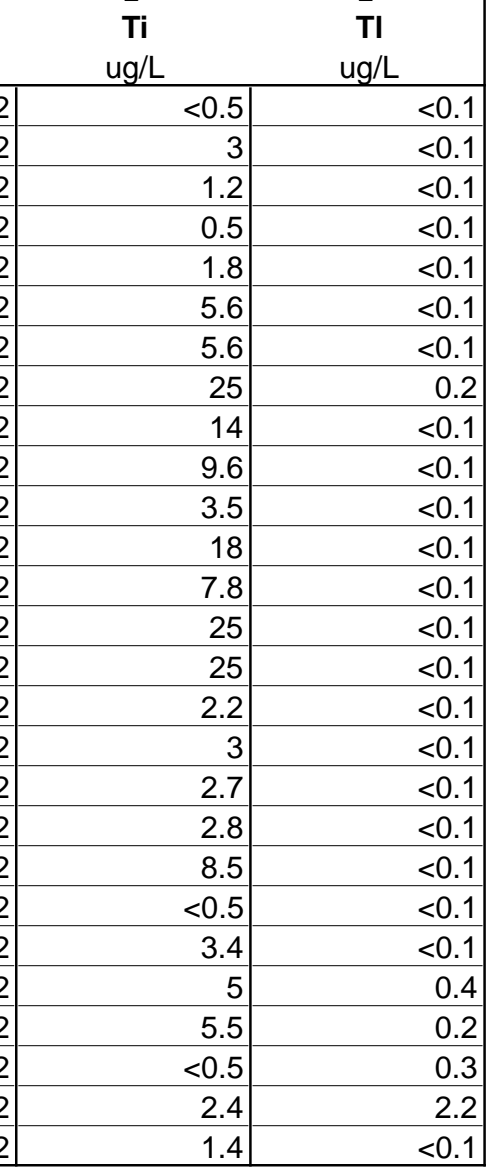




\begin{tabular}{|c|c|c|c|c|c|c|c|c|}
\hline \multicolumn{9}{|l|}{ Table 6.} \\
\hline \multicolumn{9}{|c|}{ 6a. Blank, leachate concentrations } \\
\hline $\begin{array}{c}\mathrm{NA}=\text { not analyzed } \\
\text { Field ID }\end{array}$ & Analysis Description & $\begin{array}{c}\text { ICPMS_LEACH } \\
\mathrm{Tm} \\
\mathrm{ug} / \mathrm{L} \\
\end{array}$ & $\begin{array}{c}\text { ICPMS_LEACH } \\
\mathbf{U} \\
\text { ug/L } \\
\end{array}$ & $\begin{array}{c}\text { ICPMS_LEACH } \\
\mathbf{V} \\
\mathrm{ug} / \mathrm{L} \\
\end{array}$ & $\begin{array}{c}\text { ICPMS_LEACH } \\
\text { W } \\
u g / L \\
\end{array}$ & $\begin{array}{c}\text { ICPMS_LEACH } \\
\mathbf{Y} \\
\mathrm{ug} / \mathrm{L} \\
\end{array}$ & $\begin{array}{c}\text { ICPMS_LEACH } \\
\text { Yb } \\
\text { ug/L } \\
\end{array}$ & $\begin{array}{c}\text { ICPMS_LEACH } \\
\text { Zn } \\
\text { ug/L } \\
\end{array}$ \\
\hline Blank USGS TCLP & Modified TCLP & $<0.005$ & $<0.1$ & $<0.5$ & $<0.5$ & 0.04 & 0.03 & 283 \\
\hline Chalmette 1-USGS & Modified TCLP & $<0.005$ & $<0.1$ & 3.5 & $<0.5$ & 0.2 & 0.02 & 574 \\
\hline Chalmette1D-USGS & Modified TCLP & $<0.005$ & $<0.1$ & 3.3 & $<0.5$ & 0.2 & 0.03 & 443 \\
\hline Chalmette 2-USGS & Modified TCLP & 0.01 & 0.5 & 5.7 & $<0.5$ & 1.2 & 0.08 & 1140 \\
\hline Chalmette2D-USGS & Modified TCLP & 0.02 & 0.4 & 4.9 & $<0.5$ & 1.1 & 0.1 & 1000 \\
\hline BlkUSGS SimBrac & Simulated brackish water & $<0.005$ & $<0.1$ & 0.8 & $<0.5$ & 0.03 & 0.01 & 72 \\
\hline Chalm 1-USGS & Simulated brackish water & $<0.005$ & $<0.1$ & 0.8 & $<0.5$ & 0.05 & 0.03 & 212 \\
\hline Chalm 1D-USGS & Simulated brackish water & $<0.005$ & 0.7 & 3.3 & $<0.5$ & 0.2 & $<0.005$ & 208 \\
\hline Chalm 2-USGS & Simulated brackish water & $<0.005$ & 0.7 & 1.3 & $<0.5$ & 0.05 & $<0.005$ & 135 \\
\hline Chalm 2D-USGS & Simulated brackish water & $<0.005$ & 0.7 & 1.2 & $<0.5$ & 0.09 & 0.005 & 128 \\
\hline Blank USGS SPLP & Modified SPLP & $<0.005$ & $<0.1$ & $<0.5$ & $<0.5$ & $<0.01$ & 0.01 & 4 \\
\hline Chalme 1-USGS & Modified SPLP & 0.005 & $<0.1$ & 1.9 & $<0.5$ & 0.3 & 0.04 & 101 \\
\hline Chalme 1D-USGS & Modified SPLP & $<0.005$ & $<0.1$ & 1.3 & $<0.5$ & 0.1 & 0.02 & 15 \\
\hline Chalmette 2USGS & Modified SPLP & 0.008 & 0.3 & 2.4 & $<0.5$ & 0.4 & 0.02 & 26 \\
\hline Chalm 2DUSGS & Modified SPLP & $<0.005$ & 0.4 & 1.8 & $<0.5$ & 0.3 & 0.06 & 3.6 \\
\hline Blank USGS FLT & USGS FLT & $<0.005$ & $<0.1$ & $<0.5$ & $<0.5$ & $<0.01$ & $<0.005$ & 4.2 \\
\hline Chalm 1USGS & USGS FLT & $<0.005$ & $<0.1$ & 0.6 & $<0.5$ & 0.02 & $<0.005$ & 70 \\
\hline Chalm1D-USGS & USGS FLT & $<0.005$ & $<0.1$ & 1.5 & $<0.5$ & 0.06 & 0.01 & 94 \\
\hline Chalm2-USGS & USGS FLT & $<0.005$ & $<0.1$ & 0.5 & $<0.5$ & 0.05 & 0.02 & 50 \\
\hline Chalm2D-USGS & USGS FLT & $<0.005$ & 0.5 & 2.8 & $<0.5$ & 0.2 & 0.01 & 37 \\
\hline Blank NEIC-TCLP & TCLP 1311 & $<0.005$ & $<0.1$ & $<0.5$ & $<0.5$ & $<0.01$ & 0.01 & 125 \\
\hline Chalmette1-NEIC & TCLP 1311 & $<0.005$ & $<0.1$ & 6.9 & $<0.5$ & 0.2 & 0.05 & 352 \\
\hline Chalmette1D-NEIC & TCLP 1311 duplicate & $<0.005$ & $<0.1$ & 6.9 & $<0.5$ & 0.2 & 0.03 & 336 \\
\hline Chalmette2-NEIC & TCLP 1311 & 0.02 & 1.2 & 2.7 & $<0.5$ & 1.8 & 0.1 & 3620 \\
\hline Chalmette2D-NEIC & TCLP 1311 duplicate & 0.02 & 1.1 & 2.6 & $<0.5$ & 1.8 & 0.1 & 3720 \\
\hline Ref. Standard & Standard T-103 & $<0.005$ & 6.5 & 39 & $<0.5$ & 0.04 & $<0.005$ & 22 \\
\hline Ref. Standard2 & Standard M-112 & $<0.005$ & 0.3 & 8 & 1.8 & $<0.01$ & $<0.005$ & $<0.5$ \\
\hline
\end{tabular}




\begin{tabular}{|c|c|c|}
\hline Table 6. & & \\
\hline 6a. Blank, leachate conc & rations & \\
\hline $\mathrm{NA}=$ not analyzed & & ICPMS_LEACH \\
\hline Field ID & Analysis Description & $\begin{array}{c}\bar{Z} \mathbf{Z} \\
\text { ug/L } \\
\end{array}$ \\
\hline Blank USGS TCLP & Modified TCLP & $<0.2$ \\
\hline Chalmette 1-USGS & Modified TCLP & 0.2 \\
\hline Chalmette1D-USGS & Modified TCLP & 0.8 \\
\hline Chalmette 2-USGS & Modified TCLP & $<0.2$ \\
\hline Chalmette2D-USGS & Modified TCLP & 0.6 \\
\hline BlkUSGS SimBrac & Simulated brackish water & $<0.2$ \\
\hline Chalm 1-USGS & Simulated brackish water & $<0.2$ \\
\hline Chalm 1D-USGS & Simulated brackish water & $<0.2$ \\
\hline Chalm 2-USGS & Simulated brackish water & $<0.2$ \\
\hline Chalm 2D-USGS & Simulated brackish water & $<0.2$ \\
\hline Blank USGS SPLP & Modified SPLP & $<0.2$ \\
\hline Chalme 1-USGS & Modified SPLP & 0.4 \\
\hline Chalme 1D-USGS & Modified SPLP & 0.3 \\
\hline Chalmette 2USGS & Modified SPLP & 1.0 \\
\hline Chalm 2DUSGS & Modified SPLP & 1.1 \\
\hline Blank USGS FLT & USGS FLT & $<0.2$ \\
\hline Chalm 1USGS & USGS FLT & $<0.2$ \\
\hline Chalm1D-USGS & USGS FLT & $<0.2$ \\
\hline Chalm2-USGS & USGS FLT & 0.4 \\
\hline Chalm2D-USGS & USGS FLT & 0.4 \\
\hline Blank NEIC-TCLP & TCLP 1311 & $<0.2$ \\
\hline Chalmette1-NEIC & TCLP 1311 & $<0.2$ \\
\hline Chalmette1D-NEIC & TCLP 1311 duplicate & 0.2 \\
\hline Chalmette2-NEIC & TCLP 1311 & 0.2 \\
\hline Chalmette2D-NEIC & TCLP 1311 duplicate & $<0.2$ \\
\hline Ref. Standard & Standard T-103 & 0.2 \\
\hline Ref. Standard2 & Standard M-112 & $<0.2$ \\
\hline
\end{tabular}




\section{6b. Data recalculated as}

$\mathrm{mg}$ leached per $\mathrm{kg}$ of solid

(wet weight)

\section{$\mathrm{NA}=$ not analyzed}

Field ID

\section{Analysis Description}

\section{pH \\ Spec. conduct.}

Alkalinity

$\mathrm{CaCO} 3$

IC-Aq

Blank USGS TCLP

Chalmette 1-USGS

Chalmette1D-USGS

Chalmette 2-USGS

Chalmette2D-USGS

BlkUSGS SimBrac

Chalm 1-USGS

Chalm 1D-USGS

Chalm 2-USGS

Chalm 2D-USGS

Blank USGS SPLP

Chalme 1-USGS

Chalme 1D-USGS

Chalmette 2USGS

Chalm 2DUSGS

Blank USGS FLT

Chalm 1USGS

Chalm1D-USGS

Chalm2-USGS

Chalm2D-USGS

Blank NEIC-TCLP

Chalmette1-NEIC

Chalmette1D-NEIC

Chalmette2-NEIC

Modified TCLP

Modified TCLP

Modified TCLP

Modified TCLP

Modified TCLP

Simulated brackish water

Simulated brackish water

Simulated brackish water

Simulated brackish water

Simulated brackish water

Modified SPLP

Modified SPLP

Modified SPLP

Modified SPLP

Modified SPLP

USGS FLT

USGS FLT

USGS FLT

USGS FLT

USGS FLT

TCLP EPA-NEIC

TCLP EPA-NEIC

TCLP EPA-NEIC-duplicate

Chalmette2D-NEIC

TCLP EPA-NEIC

TCLP EPA-NEIC-duplicate

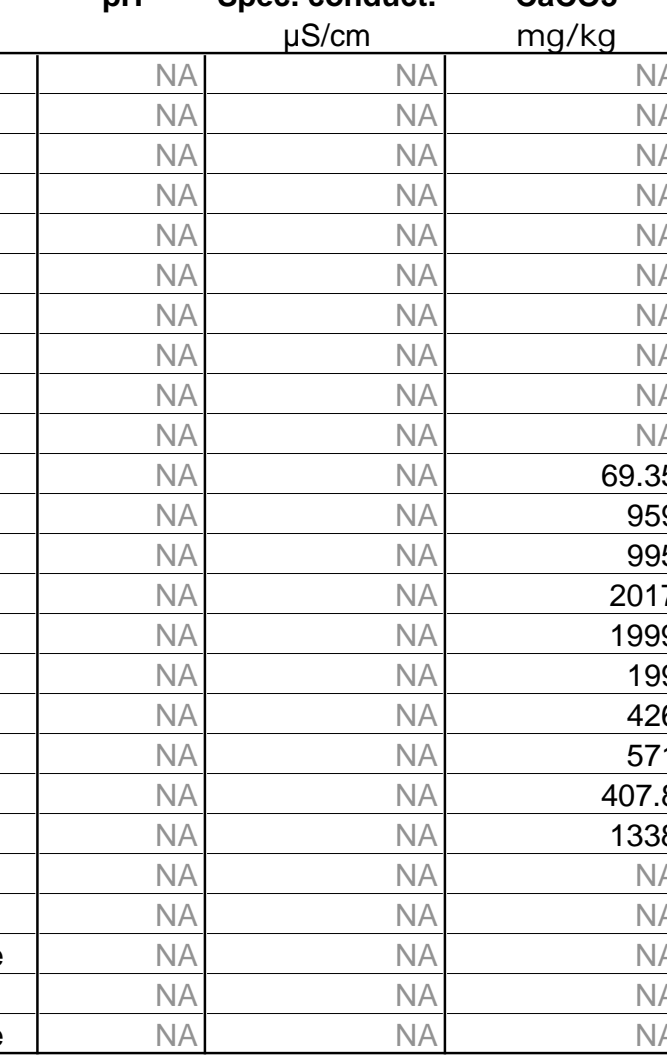

$\mathrm{mg} / \mathrm{kg}$

IC-A
$\mathbf{F g}$

\section{F}

IC-Aq

NO3

IC-Aq

SO4

$\mathrm{mg} / \mathrm{kg}$

$\mathrm{mg} / \mathrm{kg}$

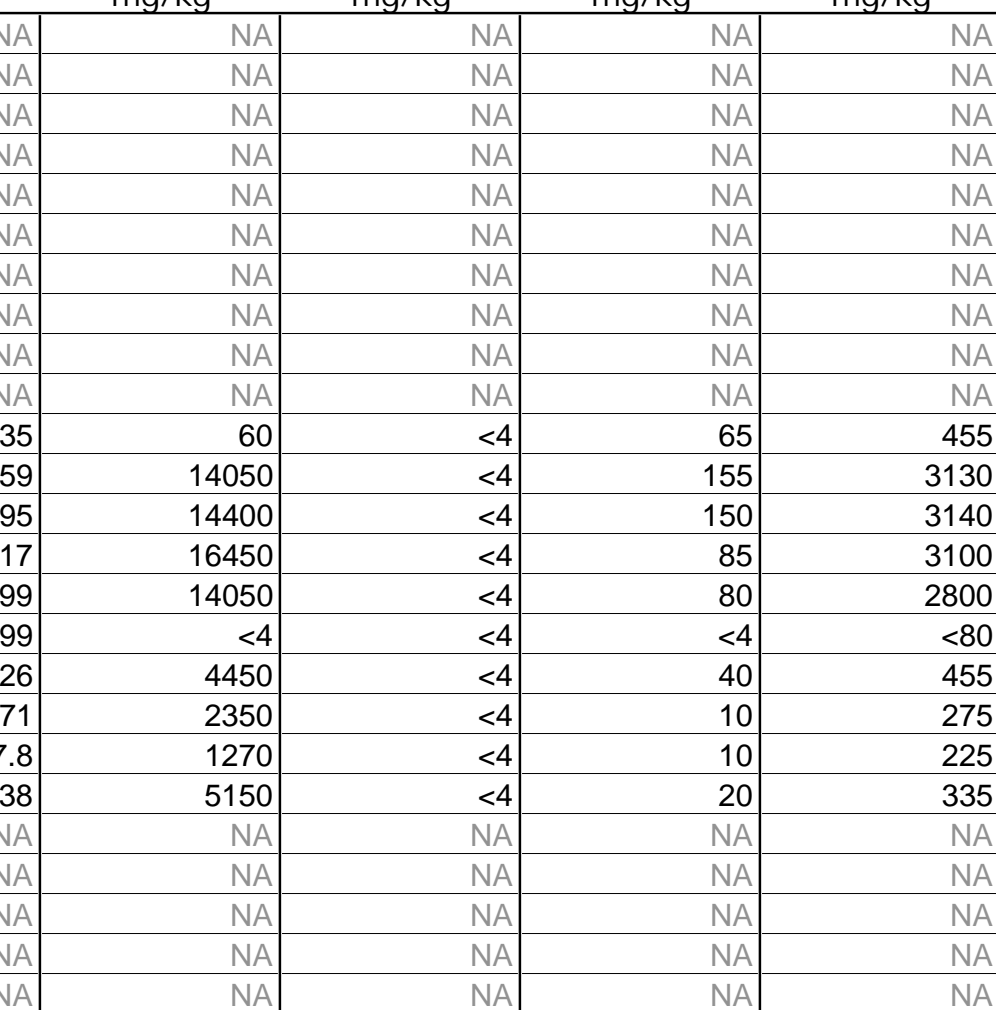


6b. Data recalculated as $\mathrm{mg}$ leached per $\mathrm{kg}$ of solid (wet weight) NA = not analyzed
Field ID

\section{Analysis Description}

Blank USGS TCLP

Chalmette 1-USGS

Chalmette1D-USGS

Chalmette 2-USGS

Chalmette2D-USGS

BlkUSGS SimBrac

Chalm 1-USGS

Chalm 1D-USGS

Chalm 2-USGS

Chalm 2D-USGS

Blank USGS SPLP

Chalme 1-USGS

Chalme 1D-USGS

Chalmette 2USGS

Chalm 2DUSGS

Blank USGS FLT

Chalm 1USGS

Chalm1D-USGS

Chalm2-USGS

Chalm2D-USGS

Blank NEIC-TCLP

Chalmette1-NEIC

Chalmette1D-NEIC

Chalmette2-NEIC

Chalmette2D-NEIC

\begin{tabular}{|c|c|c|c|}
\hline & $\mathrm{mg} / \mathrm{kg}$ & $\mathrm{mg} / \mathrm{kg}$ & $\mathrm{mg} / \mathrm{kg}$ \\
\hline Modified TCLP & 350 & $<0.06$ & 0 . \\
\hline Modified TCLP & 1350 & $<0.06$ & 1. \\
\hline Modified TCLP & 1500 & $<0.06$ & 1. \\
\hline Modified TCLP & 1400 & $<0.06$ & 1. \\
\hline Modified TCLP & 1350 & $<0.06$ & 1. \\
\hline Simulated brackish water & 18700 & $<0.06$ & 0.0 \\
\hline Simulated brackish water & 20350 & $<0.06$ & 0 . \\
\hline Simulated brackish water & 21200 & $<0.06$ & 1. \\
\hline Simulated brackish water & 21400 & $<0.06$ & 0. \\
\hline Simulated brackish water & 21550 & $<0.06$ & 0 . \\
\hline Modified SPLP & 1100 & $<0.06$ & 0 . \\
\hline Modified SPLP & 3500 & $<0.06$ & 3. \\
\hline Modified SPLP & 3150 & $<0.06$ & 3. \\
\hline Modified SPLP & 3350 & $<0.06$ & 4. \\
\hline Modified SPLP & 3250 & $<0.06$ & 5. \\
\hline USGS FLT & 750 & $<0.06$ & 0.0 \\
\hline USGS FLT & 850 & $<0.06$ & 0 . \\
\hline USGS FLT & 1300 & $<0.06$ & 1. \\
\hline USGS FLT & 1100 & $<0.06$ & 1. \\
\hline USGS FLT & 1500 & $<0.06$ & 4. \\
\hline TCLP EPA-NEIC & 700 & $<0.06$ & 0. \\
\hline TCLP EPA-NEIC & 1250 & $<0.06$ & 3. \\
\hline TCLP EPA-NEIC-duplicate & 1350 & $<0.06$ & 2. \\
\hline TCLP EPA-NEIC & 1700 & $<0.06$ & 3. \\
\hline LP EPA-NEIC-C & 1650 & $<006$ & 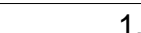 \\
\hline
\end{tabular}

(CPMS

\begin{tabular}{|c|c|c|c|c|c|c|}
\hline $\begin{array}{l}\text { MS_LEACH } \\
\text { SO4 } \\
\mathrm{mg} / \mathrm{kg}\end{array}$ & $\begin{array}{c}\text { ICPMS_LEACH } \\
\mathbf{A g} \\
\mathrm{mg} / \mathrm{kg} \\
\end{array}$ & $\begin{array}{c}\text { ICPMS_LEACH } \\
\text { Al } \\
\mathrm{mg} / \mathrm{kg} \\
\end{array}$ & $\begin{array}{c}\text { ICPMS_LEACH } \\
\text { As } \\
\mathrm{mg} / \mathrm{kg} \\
\end{array}$ & $\begin{array}{c}\text { ICPMS_LEACH } \\
\text { Ba } \\
\mathrm{mg} / \mathrm{kg} \\
\end{array}$ & $\begin{array}{c}\text { ICPMS_LEACH } \\
\text { Be } \\
\mathrm{mg} / \mathrm{kg} \\
\end{array}$ & $\begin{array}{c}\text { ICPMS_LEACH } \\
\mathbf{B i} \\
\mathrm{mg} / \mathrm{kg} \\
\end{array}$ \\
\hline 350 & $<0.06$ & 0.1 & 0.07 & 4.8 & 0.002 & 0.004 \\
\hline 1350 & $<0.06$ & 1.6 & 0.3 & 7.2 & 0.004 & $<0.004$ \\
\hline 1500 & $<0.06$ & 1.3 & 0.3 & 5.6 & 0.001 & $<0.004$ \\
\hline 1400 & $<0.06$ & 1.1 & 0.7 & 7.7 & 0.008 & $<0.004$ \\
\hline 1350 & $<0.06$ & 1.2 & 0.6 & 7.6 & 0.01 & $<0.004$ \\
\hline 18700 & $<0.06$ & 0.07 & 0.2 & 2.1 & $<0.001$ & $<0.004$ \\
\hline 20350 & $<0.06$ & 0.4 & 0.2 & 3.8 & $<0.001$ & $<0.004$ \\
\hline 21200 & $<0.06$ & 1.2 & 0.2 & 3.8 & $<0.001$ & $<0.004$ \\
\hline 21400 & $<0.06$ & 0.4 & 0.2 & 3.2 & $<0.001$ & $<0.004$ \\
\hline 21550 & $<0.06$ & 0.2 & 0.2 & 3.1 & $<0.001$ & $<0.004$ \\
\hline 1100 & $<0.06$ & 0.2 & $<0.02$ & 0.05 & $<0.001$ & $<0.004$ \\
\hline 3500 & $<0.06$ & 3.7 & $<0.02$ & 3.0 & $<0.001$ & $<0.004$ \\
\hline 3150 & $<0.06$ & 3.8 & 0.04 & 0.3 & $<0.001$ & $<0.004$ \\
\hline 3350 & $<0.06$ & 4.2 & 0.04 & 1.1 & $<0.001$ & $<0.004$ \\
\hline 3250 & $<0.06$ & 5.3 & 0.02 & 0.5 & $<0.001$ & $<0.004$ \\
\hline 750 & $<0.06$ & 0.06 & $<0.02$ & 0.4 & $<0.001$ & $<0.004$ \\
\hline 850 & $<0.06$ & 0.8 & $<0.02$ & 3.2 & $<0.001$ & $<0.004$ \\
\hline 1300 & $<0.06$ & 1.3 & $<0.02$ & 3.8 & $<0.001$ & $<0.004$ \\
\hline 1100 & $<0.06$ & 1.0 & $<0.02$ & 3.7 & $<0.001$ & $<0.004$ \\
\hline 1500 & $<0.06$ & 4.2 & 0.04 & 0.4 & $<0.001$ & $<0.004$ \\
\hline 700 & $<0.06$ & 0.3 & $<0.02$ & 1.9 & $<0.001$ & $<0.004$ \\
\hline 1250 & $<0.06$ & 3.0 & 0.4 & 5.2 & 0.001 & $<0.004$ \\
\hline 1350 & $<0.06$ & 2.3 & 0.4 & 5.3 & 0.004 & $<0.004$ \\
\hline 1700 & $<0.06$ & 3.9 & 0.7 & 8.1 & 0.02 & $<0.004$ \\
\hline 1650 & $<0.06$ & 1.8 & 0.7 & 8.8 & 0.02 & $<0.004$ \\
\hline
\end{tabular}


6b. Data recalculated as $\mathrm{mg}$ leached per $\mathrm{kg}$ of solid (wet weight)

\section{NA = not analyzed} Field ID

\section{Analysis Description}

Blank USGS TCLP

Chalmette 1-USGS

Chalmette1D-USGS

Chalmette 2-USGS

Chalmette2D-USGS

BlkUSGS SimBrac

Chalm 1-USGS

Chalm 1D-USGS

Chalm 2-USGS

Chalm 2D-USGS

Blank USGS SPLP

Chalme 1-USGS

Chalme 1D-USGS

Chalmette 2USGS

Chalm 2DUSGS

Blank USGS FLT

Chalm 1USGS

Chalm1D-USGS

Chalm2-USGS

Chalm2D-USGS

Blank NEIC-TCLP

Chalmette1-NEIC

Chalmette1D-NEIC

Chalmette2-NEIC

Chalmette2D-NEIC

\begin{tabular}{|c|c|c|c|c|c|c|c|}
\hline Analysis Description & $\begin{array}{c}\mathrm{Ca} \\
\mathrm{mg} / \mathrm{kg}\end{array}$ & $\begin{array}{c}\mathbf{C d} \\
\mathrm{mg} / \mathrm{kg}\end{array}$ & $\begin{array}{c}\mathrm{Ce} \\
\mathrm{mg} / \mathrm{kg}\end{array}$ & $\begin{array}{c}\text { Co } \\
\mathrm{mg} / \mathrm{kg}\end{array}$ & $\begin{array}{c}\mathrm{Cr} \\
\mathrm{mg} / \mathrm{kg}\end{array}$ & $\begin{array}{c}\text { Cs } \\
\mathrm{mg} / \mathrm{kg}\end{array}$ & $\begin{array}{c}\mathrm{Cu} \\
\mathrm{mg} / \mathrm{kg}\end{array}$ \\
\hline Modified TCLP & 19 & 0.002 & 0.0002 & 0.001 & $<0.02$ & 0.0004 & 0.04 \\
\hline Modified TCLP & 1460 & 0.002 & 0.007 & 0.07 & 0.07 & 0.0004 & 0.07 \\
\hline Modified TCLP & 1460 & 0.002 & 0.006 & 0.09 & 0.06 & $<0.0004$ & 0.04 \\
\hline Modified TCLP & 5200 & 0.001 & 0.03 & 0.3 & 0.2 & 0.0004 & 0.04 \\
\hline Modified TCLP & 4070 & $<0.0004$ & 0.03 & 0.2 & 0.08 & 0.0006 & 0.04 \\
\hline Simulated brackish water & 2590 & 0.01 & $<0.0002$ & 0.002 & 0.09 & 0.0024 & 0.09 \\
\hline Simulated brackish water & 3145 & $<0.0004$ & 0.001 & 0.002 & 0.07 & 0.0004 & 0.07 \\
\hline Simulated brackish water & 3255 & 0.0008 & 0.001 & 0.003 & 0.03 & $<0.0004$ & 0.10 \\
\hline Simulated brackish water & 3830 & 0.001 & 0.0004 & 0.006 & 0.04 & $<0.0004$ & 0.06 \\
\hline Simulated brackish water & 3945 & $<0.0004$ & 0.0004 & 0.006 & 0.04 & $<0.0004$ & 0.06 \\
\hline Modified SPLP & 19 & 0.0006 & $<0.0002$ & 0.0008 & 0.03 & $<0.0004$ & $<0.01$ \\
\hline Modified SPLP & 412 & 0.003 & 0.02 & 0.006 & 0.2 & $<0.0004$ & 0.2 \\
\hline Modified SPLP & 346 & $<0.0004$ & 0.009 & 0.002 & 0.04 & $<0.0004$ & 0.03 \\
\hline Modified SPLP & 595 & 0.0004 & 0.03 & 0.005 & 0.06 & $<0.0004$ & 0.03 \\
\hline Modified SPLP & 565 & 0.0004 & 0.03 & 0.007 & 0.05 & $<0.0004$ & 0.02 \\
\hline USGS FLT & $<10$ & $<0.0004$ & $<0.0002$ & $<0.0004$ & $<0.02$ & $<0.0004$ & $<0.01$ \\
\hline USGS FLT & 104 & $<0.0004$ & 0.001 & 0.0008 & $<0.02$ & $<0.0004$ & $<0.01$ \\
\hline USGS FLT & 151 & $<0.0004$ & 0.003 & 0.0086 & $<0.02$ & $<0.0004$ & 0.03 \\
\hline USGS FLT & 157 & $<0.0004$ & 0.003 & 0.001 & $<0.02$ & $<0.0004$ & 0.01 \\
\hline USGS FLT & 327 & 0.003 & 0.02 & 0.004 & $<0.02$ & $<0.0004$ & 0.04 \\
\hline TCLP EPA-NEIC & $<10$ & 0.0006 & $<0.0002$ & 0.001 & 0.03 & $<0.0004$ & 0.1 \\
\hline TCLP EPA-NEIC & 1485 & 0.004 & 0.005 & 0.07 & 0.06 & $<0.0004$ & 0.05 \\
\hline TCLP EPA-NEIC-duplicate & 1540 & 0.004 & 0.005 & 0.07 & 0.08 & $<0.0004$ & 0.09 \\
\hline TCLP EPA-NEIC & 4130 & 0.1 & 0.04 & 0.3 & 0.10 & 0.0004 & 0.2 \\
\hline TCLP EPA-NEIC-duplicate & 4215 & 0.1 & 0.04 & 0.3 & 0.09 & $<0.0004$ & 0.2 \\
\hline
\end{tabular}

ICPMS LEACH 
6b. Data recalculated as $\mathrm{mg}$ leached per $\mathrm{kg}$ of solid (wet weight)

\section{NA = not analyzed} Field ID

\section{Analysis Description}

Blank USGS TCLP

Chalmette 1-USGS

Chalmette1D-USGS

Chalmette 2-USGS

Chalmette2D-USGS

BlkUSGS SimBrac

Chalm 1-USGS

Chalm 1D-USGS

Chalm 2-USGS

Chalm 2D-USGS

Blank USGS SPLP

Chalme 1-USGS

Chalme 1D-USGS

Chalmette 2USGS

Chalm 2DUSGS

Blank USGS FLT

Chalm 1USGS

Chalm1D-USGS

Chalm2-USGS

Chalm2D-USGS

Blank NEIC-TCLP

Chalmette1-NEIC

Chalmette1D-NEIC

Chalmette2-NEIC

Chalmette2D-NEIC

\begin{tabular}{|c|c|c|c|c|c|c|c|}
\hline & $\mathrm{mg} / \mathrm{kg}$ & $\mathrm{mg} / \mathrm{kg}$ & $\mathrm{mg} / \mathrm{kg}$ & $\mathrm{mg} / \mathrm{kg}$ & $\mathrm{mg} / \mathrm{kg}$ & $\mathrm{mg} / \mathrm{kg}$ & $\mathrm{mg} / \mathrm{kg}$ \\
\hline Modified TCLP & 0.005 & $<0.0001$ & $<0.0001$ & $<1$ & $<0.001$ & $<0.0001$ & $<0.001$ \\
\hline Modified TCLP & $<0.0001$ & 0.0001 & 0.0006 & $<1$ & $<0.001$ & 0.001 & $<0.001$ \\
\hline Modified TCLP & 0.0006 & 0.0004 & 0.0002 & $<1$ & 0.001 & 0.0008 & 0.001 \\
\hline Modified TCLP & 0.003 & 0.002 & 0.002 & 544 & 0.002 & 0.006 & 0.002 \\
\hline Modified TCLP & 0.004 & 0.002 & 0.002 & 590 & 0.002 & 0.005 & $<0.001$ \\
\hline Simulated brackish water & $<0.0001$ & $<0.0001$ & $<0.0001$ & $<1$ & $<0.001$ & 0.0002 & $<0.001$ \\
\hline Simulated brackish water & $<0.0001$ & 0.0002 & $<0.0001$ & $<1$ & $<0.001$ & 0.0001 & $<0.001$ \\
\hline Simulated brackish water & $<0.0001$ & 0.0001 & $<0.0001$ & $<1$ & 0.0014 & 0.0006 & $<0.001$ \\
\hline Simulated brackish water & $<0.0001$ & $<0.0001$ & $<0.0001$ & $<1$ & $<0.001$ & 0.0006 & $<0.001$ \\
\hline Simulated brackish water & $<0.0001$ & 0.0002 & $<0.0001$ & $<1$ & $<0.001$ & 0.0002 & $<0.001$ \\
\hline Modified SPLP & $<0.0001$ & $<0.0001$ & 0.0002 & $<1$ & $<0.001$ & 0.0004 & $<0.001$ \\
\hline Modified SPLP & 0.0008 & 0.0002 & 0.0006 & $<1$ & $<0.001$ & 0.002 & $<0.001$ \\
\hline Modified SPLP & 0.0006 & 0.0001 & 0.0002 & $<1$ & 0.001 & 0.0008 & $<0.001$ \\
\hline Modified SPLP & 0.002 & 0.004 & 0.0004 & $<1$ & 0.002 & 0.003 & $<0.001$ \\
\hline Modified SPLP & 0.001 & 0.001 & 0.0006 & $<1$ & 0.002 & 0.002 & $<0.001$ \\
\hline USGS FLT & $<0.0001$ & $<0.0001$ & 0.0001 & $<1$ & $<0.001$ & 0.0004 & $<0.001$ \\
\hline USGS FLT & $<0.0001$ & 0.0002 & $<0.0001$ & $<1$ & $<0.001$ & 0.0002 & $<0.001$ \\
\hline USGS FLT & $<0.0001$ & $<0.0001$ & 0.0004 & $<1$ & $<0.001$ & 0.0006 & $<0.001$ \\
\hline USGS FLT & $<0.0001$ & $<0.0001$ & 0.0002 & $<1$ & $<0.001$ & 0.001 & 0.001 \\
\hline USGS FLT & 0.001 & 0.0004 & 0.0004 & $<1$ & 0.002 & 0.002 & $<0.001$ \\
\hline TCLP EPA-NEIC & $<0.0001$ & $<0.0001$ & $<0.0001$ & $<1$ & $<0.001$ & $<0.0001$ & $<0.001$ \\
\hline TCLP EPA-NEIC & 0.0002 & 0.0002 & 0.0002 & $<1$ & $<0.001$ & 0.0008 & $<0.001$ \\
\hline TCLP EPA-NEIC-duplicate & 0.0001 & 0.0002 & 0.0004 & $<1$ & $<0.001$ & 0.0014 & 0.001 \\
\hline TCLP EPA-NEIC & 0.006 & 0.003 & 0.002 & $<1$ & 0.004 & 0.01 & 0.004 \\
\hline TCLP EPA-NEIC-duplicate & 0.004 & 0.004 & 0.002 & $<1$ & 0.002 & 0.01 & 0.004 \\
\hline
\end{tabular}


6b. Data recalculated as $\mathrm{mg}$ leached per $\mathrm{kg}$ of solid (wet weight)

\section{$\mathrm{NA}=$ not analyzed} Field ID

Blank USGS TCLP

Chalmette 1-USGS

Chalmette1D-USGS

Chalmette 2-USGS

Chalmette2D-USGS

BlkUSGS SimBrac

Chalm 1-USGS

Chalm 1D-USGS

Chalm 2-USGS

Chalm 2D-USGS

Blank USGS SPLP

Chalme 1-USGS

Chalme 1D-USGS

Chalmette 2USGS

Chalm 2DUSGS

Blank USGS FLT

Chalm 1USGS

Chalm1D-USGS

Chalm2-USGS

Chalm2D-USGS

Blank NEIC-TCLP

Chalmette1-NEIC

Chalmette1D-NEIC

Chalmette2-NEIC

Chalmette2D-NEIC

\begin{tabular}{|c|c|c|c|c|c|c|c|}
\hline Analysis Description & $\begin{array}{c}\text { CVAFS } \\
\mathrm{Hg} \\
\mathrm{mg} / \mathrm{Kg}\end{array}$ & $\begin{array}{c}\text { ICPMS_LEACH } \\
\text { Ho } \\
\mathrm{mg} / \mathrm{kg}\end{array}$ & $\begin{array}{c}\text { ICPMS_LEACH } \\
\mathbf{K} \\
\mathrm{mg} / \mathrm{kg}\end{array}$ & $\begin{array}{c}\text { ICPMS_LEACH } \\
\text { La } \\
\mathrm{mg} / \mathrm{kg}\end{array}$ & $\begin{array}{c}\text { ICPMS_LEACH } \\
\text { Li } \\
\mathrm{mg} / \mathrm{kg}\end{array}$ & $\begin{array}{c}\text { ICPMS_LEACH } \\
\text { Lu } \\
\mathrm{mg} / \mathrm{kg}\end{array}$ & $\begin{array}{c}\text { ICPMS_LEACH } \\
\mathbf{M g} \\
\mathrm{mg} / \mathrm{kg}\end{array}$ \\
\hline Modified TCLP & $<0.0003$ & $<0.0001$ & 117 & 0.0002 & 0.1 & $<0.002$ & 0.001 \\
\hline Modified TCLP & $<0.0003$ & 0.0002 & 775 & 0.003 & 0.5 & $<0.002$ & 0.8 \\
\hline Modified TCLP & $<0.0003$ & 0.0001 & 750 & 0.003 & 0.4 & $<0.002$ & 0.8 \\
\hline Modified TCLP & $<0.0003$ & 0.001 & 1230 & 0.02 & 0.3 & $<0.002$ & 1.1 \\
\hline Modified TCLP & $<0.0003$ & 0.0006 & 1210 & 0.01 & 0.3 & $<0.002$ & 1.0 \\
\hline Simulated brackish water & $<0.0003$ & $<0.0001$ & 2760 & 0.0002 & 30 & $<0.002$ & 3.0 \\
\hline Simulated brackish water & $<0.0003$ & $<0.0001$ & 2990 & 0.0008 & 27 & $<0.002$ & 3.4 \\
\hline Simulated brackish water & $<0.0003$ & $<0.0001$ & 3075 & 0.0006 & 26 & $<0.002$ & 3.7 \\
\hline Simulated brackish water & 0.001 & $<0.0001$ & 3255 & 0.0004 & 25 & $<0.002$ & 3.5 \\
\hline Simulated brackish water & $<0.0003$ & $<0.0001$ & 3295 & 0.0002 & 26 & $<0.002$ & 3.6 \\
\hline Modified SPLP & $<0.0003$ & $<0.0001$ & 10 & 0.0004 & 0.2 & $<0.002$ & 0.004 \\
\hline Modified SPLP & $<0.0003$ & 0.0002 & 499 & 0.009 & $<0.018$ & $<0.002$ & 0.3 \\
\hline Modified SPLP & $<0.0003$ & 0.0002 & 467 & 0.005 & $<0.018$ & $<0.002$ & 0.3 \\
\hline Modified SPLP & $<0.0003$ & 0.0004 & 735 & 0.01 & $<0.018$ & $<0.002$ & 0.3 \\
\hline Modified SPLP & $<0.0003$ & 0.0006 & 710 & 0.01 & $<0.018$ & $<0.002$ & 0.3 \\
\hline USGS FLT & $<0.0003$ & $<0.0001$ & 3.5 & $<0.0002$ & $<0.018$ & $<0.002$ & 0.0004 \\
\hline USGS FLT & $<0.0003$ & $<0.0001$ & 119 & 0.0008 & $<0.018$ & $<0.002$ & 0.08 \\
\hline USGS FLT & $<0.0003$ & $<0.0001$ & 209 & 0.002 & $<0.018$ & $<0.002$ & 0.1 \\
\hline USGS FLT & $<0.0003$ & $<0.0001$ & 133 & 0.002 & $<0.018$ & $<0.002$ & 0.08 \\
\hline USGS FLT & $<0.0003$ & 0.0002 & 570 & 0.01 & 0.02 & $<0.002$ & 0.2 \\
\hline TCLP EPA-NEIC & $<0.0003$ & $<0.0001$ & 18 & 0.0002 & 0.2 & $<0.002$ & 0.0006 \\
\hline TCLP EPA-NEIC & $<0.0003$ & 0.0001 & 685 & 0.003 & 0.1 & $<0.002$ & 0.8 \\
\hline TCLP EPA-NEIC-duplicate & $<0.0003$ & 0.0001 & 715 & 0.003 & 0.2 & $<0.002$ & 0.8 \\
\hline TCLP EPA-NEIC & $<0.0003$ & 0.001 & 1190 & 0.02 & 0.1 & $<0.002$ & 1.1 \\
\hline TCLP EPA-NEIC-duplicate & $<0.0003$ & 0.001 & 1190 & 0.02 & 0.2 & $<0.002$ & 1.1 \\
\hline
\end{tabular}


6b. Data recalculated as $\mathrm{mg}$ leached per $\mathrm{kg}$ of solid

(wet weight)

NA = not analyzed

Field ID

\section{Analysis Description}

Blank USGS TCLP

Chalmette 1-USGS

Chalmette1D-USGS

Chalmette 2-USGS

Chalmette2D-USGS

BlkUSGS SimBrac

Chalm 1-USGS

Chalm 1D-USGS

Chalm 2-USGS

Chalm 2D-USGS

Blank USGS SPLP

Chalme 1-USGS

Chalme 1D-USGS

Chalmette 2USGS

Chalm 2DUSGS

Blank USGS FLT

Chalm 1USGS

Chalm1D-USGS

Chalm2-USGS

Chalm2D-USGS

Blank NEIC-TCLP

Chalmette1-NEIC

Chalmette1D-NEIC

Chalmette2-NEIC

Chalmette2D-NEIC

\begin{tabular}{|c|c|c|c|c|c|c|c|}
\hline & $\mathrm{mg} / \mathrm{kg}$ & $\mathrm{mg} / \mathrm{kg}$ & $\mathrm{mg} / \mathrm{kg}$ & $\mathrm{mg} / \mathrm{kg}$ & $\mathrm{mg} / \mathrm{kg}$ & $\mathrm{mg} / \mathrm{kg}$ & $\mathrm{mg} / \mathrm{kg}$ \\
\hline Modified TCLP & $<0.004$ & 0.1 & 70000 & $<0.004$ & 0.0004 & 0.03 & $<0.5$ \\
\hline Modified TCLP & 27 & 0.06 & 73500 & $<0.004$ & 0.002 & 0.09 & 15 \\
\hline Modified TCLP & 26 & $<0.04$ & 71000 & $<0.004$ & 0.004 & 0.10 & 15 \\
\hline Modified TCLP & 150 & $<0.04$ & 72000 & $<0.004$ & 0.02 & 0.7 & 5 \\
\hline Modified TCLP & 139 & $<0.04$ & 71000 & $<0.004$ & 0.02 & 0.6 & 10 \\
\hline Simulated brackish water & 0.2 & $<0.04$ & 85500 & $<0.004$ & 0.0004 & 0.07 & 2.5 \\
\hline Simulated brackish water & 0.08 & 0.1 & 90000 & $<0.004$ & 0.0004 & 0.02 & 2.0 \\
\hline Simulated brackish water & 0.08 & 0.2 & 98500 & 0.1 & 0.001 & 0.02 & 5.0 \\
\hline Simulated brackish water & 36 & 0.2 & 95000 & $<0.004$ & 0.0006 & 0.04 & 4.0 \\
\hline Simulated brackish water & 37 & 0.1 & 97000 & $<0.004$ & $<0.0002$ & 0.05 & 2.5 \\
\hline Modified SPLP & 0.1 & $<0.04$ & 168 & $<0.004$ & $<0.0002$ & 0.08 & $<0.5$ \\
\hline Modified SPLP & 0.3 & 0.2 & 9100 & $<0.004$ & 0.009 & 0.2 & $<0.5$ \\
\hline Modified SPLP & 0.3 & 0.1 & 8400 & $<0.004$ & 0.004 & 0.09 & $<0.5$ \\
\hline Modified SPLP & 4.1 & 0.1 & 9800 & $<0.004$ & 0.02 & 0.1 & 3.5 \\
\hline Modified SPLP & 3.6 & 0.1 & 9250 & $<0.004$ & 0.01 & 0.1 & 3.0 \\
\hline USGS FLT & $<0.004$ & $<0.04$ & 59 & $<0.004$ & $<0.0002$ & $<0.008$ & $<0.5$ \\
\hline USGS FLT & 0.02 & $<0.04$ & 2435 & $<0.004$ & 0.0006 & $<0.008$ & $<0.5$ \\
\hline USGS FLT & 0.004 & $<0.04$ & 4005 & $<0.004$ & 0.0008 & $<0.008$ & $<0.5$ \\
\hline USGS FLT & 0.2 & $<0.04$ & 2130 & $<0.004$ & 0.002 & $<0.008$ & $<0.5$ \\
\hline USGS FLT & 0.8 & $<0.04$ & 8000 & $<0.004$ & 0.01 & 0.1 & $<0.5$ \\
\hline TCLP EPA-NEIC & $<0.004$ & $<0.04$ & 72500 & $<0.004$ & 0.0004 & 0.02 & $<0.5$ \\
\hline TCLP EPA-NEIC & 29 & $<0.04$ & 76000 & $<0.004$ & 0.003 & 0.1 & 20 \\
\hline TCLP EPA-NEIC-duplicate & 30 & $<0.04$ & 77500 & $<0.004$ & 0.004 & 0.1 & 20 \\
\hline TCLP EPA-NEIC & 142 & $<0.04$ & 74000 & $<0.004$ & 0.03 & 0.8 & 1.0 \\
\hline TCLP EPA-NEIC-duplicate & 145 & $<0.04$ & 74000 & $<0.004$ & 0.03 & 0.8 & $<0.5$ \\
\hline
\end{tabular}


6b. Data recalculated as $\mathrm{mg}$ leached per $\mathrm{kg}$ of solid (wet weight) NA = not analyzed
Field ID

\begin{tabular}{|c|c|c|c|}
\hline Analysis Description & $\begin{array}{c}\text { ICPMS_LEACH } \\
\mathbf{P b} \\
\mathrm{mg} / \mathrm{kg} \\
\end{array}$ & $\begin{array}{c}\text { ICPMS_LEACH } \\
\text { Pr } \\
\mathrm{mg} / \mathrm{kg} \\
\end{array}$ & $\begin{array}{c}\text { ICPMS_LEACH } \\
\mathbf{R b} \\
\mathrm{mg} / \mathrm{kg} \\
\end{array}$ \\
\hline Modified TCLP & 0.004 & $<0.0002$ & 0.0 \\
\hline Modified TCLP & 0.06 & 0.0008 & \\
\hline Modified TCLP & 0.05 & 0.0006 & \\
\hline Modified TCLP & 0.2 & 0.004 & \\
\hline Modified TCLP & 0.2 & 0.003 & 0 \\
\hline Simulated brackish water & 0.002 & $<0.0002$ & 0 \\
\hline Simulated brackish water & 0.002 & 0.0002 & 0 \\
\hline Simulated brackish water & 0.008 & 0.0002 & 0 \\
\hline Simulated brackish water & 0.004 & 0.0002 & 0 \\
\hline Simulated brackish water & 0.004 & $<0.0002$ & 0 \\
\hline Modified SPLP & 0.004 & $<0.0002$ & $<0.000$ \\
\hline Modified SPLP & 0.06 & 0.003 & 0. \\
\hline Modified SPLP & 0.008 & 0.001 & 0.0 \\
\hline Modified SPLP & 0.01 & 0.004 & 0. \\
\hline Modified SPLP & 0.01 & 0.003 & 0 . \\
\hline USGS FLT & $<0.001$ & $<0.0002$ & $<0.000$ \\
\hline USGS FLT & 0.002 & 0.0004 & 0. \\
\hline USGS FLT & 0.002 & 0.0006 & 0 \\
\hline USGS FLT & 0.002 & 0.0004 & 0. \\
\hline USGS FLT & 0.04 & 0.002 & 0.0 \\
\hline TCLP EPA-NEIC & 0.004 & $<0.0002$ & 0.000 \\
\hline TCLP EPA-NEIC & 0.07 & 0.001 & 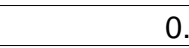 \\
\hline TCLP EPA-NEIC-duplicate & 0.07 & 0.001 & 0 \\
\hline TCLP EPA-NEIC & 0.5 & 0.006 & 0 \\
\hline TCLP EPA-NEIC-duplicate & 0.5 & 0.006 & \\
\hline
\end{tabular}

Blank USGS TCLP

Chalmette 1-USGS

Chalmette1D-USGS

Chalmette 2-USGS

Chalmette2D-USGS

BlkUSGS SimBrac

Chalm 1-USGS

Chalm 1D-USGS

Chalm 2-USGS

Chalm 2D-USGS

Blank USGS SPLP

Chalme 1-USGS

Chalme 1D-USGS

Chalmette 2USGS

Chalm 2DUSGS

Blank USGS FLT

Chalm 1USGS

Chalm1D-USGS

Chalm2-USGS

Chalm2D-USGS

Blank NEIC-TCLP

Chalmette1-NEIC

Chalmette1D-NEIC

Chalmette2-NEIC

TCLP EPA-NEIC-duplicate

0.5

0.006

\section{ICPMS_LEACH}

$\mathrm{Sb}$

ICPMS_LEACH

ICPMS_LEACH ICPMS_LEACH

$\mathrm{mg} / \mathrm{kg} \mathrm{mg} / \mathrm{kg} \quad \mathrm{Se}$

\begin{tabular}{|r|r|r|r|}
\hline & $\mathrm{mg} / \mathrm{kg}$ \\
\hline 0.006 & $<0.012$ & 0.1 & $<10$ \\
\hline
\end{tabular}

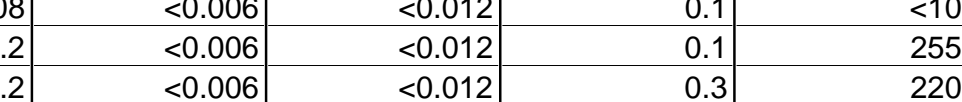

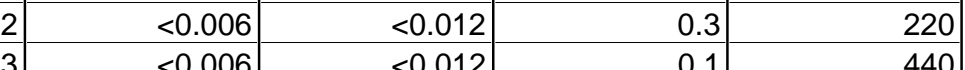

$<0.012$

$<0.006$

$<0.006$

$3<0.006$

\begin{tabular}{|l|l|l|}
\hline & $<0.006$ & $<0.012$ \\
\hline 0.3 & $<0.006$ & $<0.012$ \\
\hline
\end{tabular}

$<0.006 \quad<0.012$

$<0.006$

\begin{tabular}{l}
$<08$ \\
\hline 0.006
\end{tabular}

$<0.006$

$<0.006$

\begin{tabular}{ll}
0.1 & $<0.006$ \\
\hline
\end{tabular}

\begin{tabular}{|l|l|}
\hline & $<0.006$ \\
\hline 0.03 & $<0.006$
\end{tabular}

$<0.006$

$<0.006$
$<0.006$

\begin{tabular}{l}
$<2<0.006$ \\
\hline
\end{tabular}

$0.3<0.006$

$<0.006$

$<0.012$

$<0.012$

$<0.012$

\begin{tabular}{l}
$<0.012$ \\
\hline 0.012
\end{tabular}

$<0.012$

$<0.012$

$<0.012$

Chalmette2D-NEIC

0.3

$<0.012$

$<0.012$

$<0.012$

$<0.012$

$<0.012$

$<0.012$

\begin{tabular}{r|r|}
\hline 0.1 & 425 \\
\hline 1.0 & $<10$ \\
\hline 1.1 & 90 \\
\hline 1.1 & 75 \\
\hline 1.1 & 160 \\
\hline 1.1 & 155 \\
\hline 0.09 & $<10$ \\
\hline 0.1 & 85 \\
\hline 0.1 & 100 \\
\hline 0.2 & 195 \\
\hline 0.1 & 180 \\
\hline 0.07 & $<10$ \\
\hline 0.09 & $<10$ \\
\hline 0.1 & $<10$ \\
\hline 0.07 & $<10$ \\
\hline 0.1 & $<10$ \\
\hline 0.05 & $<10$ \\
\hline 0.2 & 215 \\
\hline 0.1 & 245 \\
\hline 0.1 & 495 \\
\hline 0.2 & 470 \\
\hline
\end{tabular}


6b. Data recalculated as $\mathrm{mg}$ leached per $\mathrm{kg}$ of solid (wet weight)

\section{NA = not analyzed} Field ID

Blank USGS TCLP

Chalmette 1-USGS

Chalmette1D-USGS

Chalmette 2-USGS

Chalmette2D-USGS

BlkUSGS SimBrac

Chalm 1-USGS

Chalm 1D-USGS

Chalm 2-USGS

Chalm 2D-USGS

Blank USGS SPLP

Chalme 1-USGS

Chalme 1D-USGS

Chalmette 2USGS

Chalm 2DUSGS

Blank USGS FLT

Chalm 1USGS

Chalm1D-USGS

Chalm2-USGS

Chalm2D-USGS

Blank NEIC-TCLP

Chalmette1-NEIC

Chalmette1D-NEIC

Chalmette2-NEIC

Chalmette2D-NEIC

\begin{tabular}{|c|c|c|c|c|c|c|c|}
\hline Analysis Description & $\begin{array}{c}\text { ICPMS_LEACH } \\
\mathrm{Sm} \\
\mathrm{mg} / \mathrm{kg}\end{array}$ & $\begin{array}{c}\text { ICPMS_LEACH } \\
\text { Sr } \\
\mathrm{mg} / \mathrm{kg}\end{array}$ & $\begin{array}{c}\text { ICPMS_LEACH } \\
\text { Ta } \\
\mathrm{mg} / \mathrm{kg}\end{array}$ & $\begin{array}{c}\text { ICPMS_LEACH } \\
\text { Tb } \\
\mathrm{mg} / \mathrm{kg}\end{array}$ & $\begin{array}{c}\text { ICPMS_LEACH } \\
\text { Th } \\
\mathrm{mg} / \mathrm{kg}\end{array}$ & $\begin{array}{c}\text { ICPMS_LEACH } \\
\mathrm{Ti} \\
\mathrm{mg} / \mathrm{kg}\end{array}$ & $\begin{array}{c}\text { ICPMS_LEACH } \\
\text { TI } \\
\mathrm{mg} / \mathrm{kg}\end{array}$ \\
\hline Modified TCLP & $<0.0002$ & 0.6 & $<0.0004$ & $<0.0001$ & $<0.004$ & $<0.01$ & $<0.002$ \\
\hline Modified TCLP & 0.001 & 6.8 & $<0.0004$ & 0.0002 & $<0.004$ & 0.06 & $<0.002$ \\
\hline Modified TCLP & $<0.0002$ & 6.7 & $<0.0004$ & $<0.0001$ & $<0.004$ & 0.02 & $<0.002$ \\
\hline Modified TCLP & 0.004 & 12 & $<0.0004$ & 0.0008 & $<0.004$ & 0.01 & $<0.002$ \\
\hline Modified TCLP & 0.004 & 10 & $<0.0004$ & 0.0006 & $<0.004$ & 0.04 & $<0.002$ \\
\hline Simulated brackish water & $<0.0002$ & 32 & $<0.0004$ & $<0.0001$ & $<0.004$ & 0.1 & $<0.002$ \\
\hline Simulated brackish water & $<0.0002$ & 28 & $<0.0004$ & $<0.0001$ & $<0.004$ & 0.1 & $<0.002$ \\
\hline Simulated brackish water & 0.002 & 29 & 0.09 & $<0.0001$ & $<0.004$ & 0.5 & 0.004 \\
\hline Simulated brackish water & $<0.0002$ & 29 & 0.01 & $<0.0001$ & $<0.004$ & 0.3 & $<0.002$ \\
\hline Simulated brackish water & $<0.0002$ & 29 & $<0.0004$ & $<0.0001$ & $<0.004$ & 0.2 & $<0.002$ \\
\hline Modified SPLP & $<0.0002$ & 0.06 & $<0.0004$ & $<0.0001$ & $<0.004$ & 0.07 & $<0.002$ \\
\hline Modified SPLP & 0.0006 & 1.9 & $<0.0004$ & 0.0004 & $<0.004$ & 0.4 & $<0.002$ \\
\hline Modified SPLP & 0.0004 & 1.7 & $<0.0004$ & 0.0001 & $<0.004$ & 0.2 & $<0.002$ \\
\hline Modified SPLP & 0.003 & 2.0 & $<0.0004$ & 0.0002 & $<0.004$ & 0.5 & $<0.002$ \\
\hline Modified SPLP & 0.003 & 2.0 & $<0.0004$ & 0.0004 & $<0.004$ & 0.5 & $<0.002$ \\
\hline USGS FLT & $<0.0002$ & $<0.01$ & $<0.0004$ & $<0.0001$ & $<0.004$ & 0.04 & $<0.002$ \\
\hline USGS FLT & $<0.0002$ & 0.5 & $<0.0004$ & $<0.0001$ & $<0.004$ & 0.06 & $<0.002$ \\
\hline USGS FLT & 0.0004 & 0.7 & $<0.0004$ & $<0.0001$ & $<0.004$ & 0.05 & $<0.002$ \\
\hline USGS FLT & $<0.0002$ & 0.6 & $<0.0004$ & $<0.0001$ & $<0.004$ & 0.06 & $<0.002$ \\
\hline USGS FLT & 0.001 & 1.2 & $<0.0004$ & 0.0001 & $<0.004$ & 0.2 & $<0.002$ \\
\hline TCLP EPA-NEIC & $<0.0002$ & 0.03 & $<0.0004$ & $<0.0001$ & $<0.004$ & $<0.01$ & $<0.002$ \\
\hline TCLP EPA-NEIC & $<0.0002$ & 6.5 & $<0.0004$ & 0.0001 & $<0.004$ & 0.07 & $<0.002$ \\
\hline TCLP EPA-NEIC-duplicate & $<0.0002$ & 6.9 & $<0.0004$ & 0.0002 & $<0.004$ & 0.1 & 0.008 \\
\hline TCLP EPA-NEIC & 0.01 & 10.6 & $<0.0004$ & 0.001 & $<0.004$ & 0.1 & 0.004 \\
\hline TCLP EPA-NEIC-duplicate & 0.006 & 10.9 & $<0.0004$ & 0.001 & $<0.004$ & $<0.01$ & 0.006 \\
\hline
\end{tabular}


6b. Data recalculated as $\mathrm{mg}$ leached per $\mathrm{kg}$ of solid (wet weight)

\section{$\mathrm{NA}=$ not analyzed} Field ID

Blank USGS TCLP

Chalmette 1-USGS

Chalmette1D-USGS

Chalmette 2-USGS

Chalmette2D-USGS

BlkUSGS SimBrac

Chalm 1-USGS

Chalm 1D-USGS

Chalm 2-USGS

Chalm 2D-USGS

Blank USGS SPLP

Chalme 1-USGS

Chalme 1D-USGS

Chalmette 2USGS

Chalm 2DUSGS

Blank USGS FLT

Chalm 1USGS

Chalm1D-USGS

Chalm2-USGS

Chalm2D-USGS

Blank NEIC-TCLP

Chalmette1-NEIC

Chalmette1D-NEIC

Chalmette2-NEIC

Chalmette2D-NEIC

\section{Analysis Description}

ICPMS_LEACH

ICPMS_LEACH

U

ICPMS_LEACH

ICPMS_LEACH

ICPMS_LEACH

ICPMS_LEACH

Yb

ICPMS_LEACH

$\mathrm{mg} / \mathrm{kg} \mathrm{mg} / \mathrm{kg} \quad \mathrm{mg} / \mathrm{kg}$

$\mathrm{mg} / \mathrm{kg} \quad \mathrm{mg} / \mathrm{kg}$

$\mathrm{mg} / \mathrm{kg}$

$\mathrm{mg} / \mathrm{kg}$

$\mathrm{mg} / \mathrm{kg}$

\begin{tabular}{|c|c|c|c|c|c|c|c|}
\hline & & & & & & & \\
\hline Modified TCLP & $<0.0001$ & $<0.002$ & $<0.01$ & $<0.01$ & 0.0008 & 0.0006 & 5.7 \\
\hline Modified TCLP & $<0.0001$ & $<0.002$ & 0.07 & $<0.01$ & 0.004 & 0.0004 & 11 \\
\hline Modified TCLP & $<0.0001$ & $<0.002$ & 0.07 & $<0.01$ & 0.003 & 0.0006 & 8.9 \\
\hline Modified TCLP & 0.0002 & 0.010 & 0.1 & $<0.01$ & 0.02 & 0.002 & 23 \\
\hline Modified TCLP & 0.0004 & 0.008 & 0.10 & $<0.01$ & 0.02 & 0.0022 & 20 \\
\hline Simulated brackish water & $<0.0001$ & $<0.002$ & 0.02 & $<0.01$ & 0.0006 & 0.0002 & 1.4 \\
\hline Simulated brackish water & $<0.0001$ & $<0.002$ & 0.02 & $<0.01$ & 0.001 & 0.0006 & 4.2 \\
\hline Simulated brackish water & $<0.0001$ & 0.01 & 0.07 & $<0.01$ & 0.004 & $<0.0001$ & 4.2 \\
\hline Simulated brackish water & $<0.0001$ & 0.01 & 0.03 & $<0.01$ & 0.001 & $<0.0001$ & 2.7 \\
\hline Simulated brackish water & $<0.0001$ & 0.01 & 0.02 & $<0.01$ & 0.002 & 0.0001 & 2.6 \\
\hline Modified SPLP & $<0.0001$ & $<0.002$ & $<0.01$ & $<0.01$ & $<0.0002$ & 0.0002 & 0.08 \\
\hline Modified SPLP & 0.0001 & $<0.002$ & 0.04 & $<0.01$ & 0.005 & 0.0008 & 2.0 \\
\hline Modified SPLP & $<0.0001$ & $<0.002$ & 0.03 & $<0.01$ & 0.002 & 0.0004 & 0.3 \\
\hline Modified SPLP & 0.0002 & 0.006 & 0.05 & $<0.01$ & 0.007 & 0.0004 & 0.5 \\
\hline Modified SPLP & $<0.0001$ & 0.007 & 0.04 & $<0.01$ & 0.006 & 0.001 & 0.07 \\
\hline USGS FLT & $<0.0001$ & $<0.002$ & $<0.01$ & $<0.01$ & $<0.0002$ & $<0.0001$ & 0.08 \\
\hline USGS FLT & $<0.0001$ & $<0.002$ & 0.01 & $<0.01$ & 0.0004 & $<0.0001$ & 1.4 \\
\hline USGS FLT & $<0.0001$ & $<0.002$ & 0.03 & $<0.01$ & 0.001 & 0.0002 & 1.9 \\
\hline USGS FLT & $<0.0001$ & $<0.002$ & 0.01 & $<0.01$ & 0.001 & 0.0004 & 1.0 \\
\hline USGS FLT & $<0.0001$ & 0.009 & 0.06 & $<0.01$ & 0.005 & 0.0002 & 0.7 \\
\hline TCLP EPA-NEIC & $<0.0001$ & $<0.002$ & $<0.01$ & $<0.01$ & $<0.0002$ & 0.0002 & 2.5 \\
\hline TCLP EPA-NEIC & $<0.0001$ & $<0.002$ & 0.1 & $<0.01$ & 0.004 & 0.001 & 7.0 \\
\hline TCLP EPA-NEIC-duplicate & $<0.0001$ & $<0.002$ & 0.1 & $<0.01$ & 0.004 & 0.0006 & 6.7 \\
\hline TCLP EPA-NEIC & 0.0004 & 0.02 & 0.05 & $<0.01$ & 0.04 & 0.003 & 72 \\
\hline TCLP EPA-NEIC-duplicate & 0.0004 & 0.02 & 0.05 & $<0.01$ & 0.04 & 0.002 & 74 \\
\hline
\end{tabular}




\begin{tabular}{|c|c|c|}
\hline \multicolumn{2}{|c|}{\begin{tabular}{|l} 
6b. Data recalculated as \\
mg leached per kg of solid \\
(wet weight) \\
$\begin{array}{c}\text { NA }=\text { not analyzed } \\
\text { Field ID }\end{array} \quad$ Analysis Description
\end{tabular}} & \multirow{2}{*}{$\begin{array}{c}\begin{array}{c}\text { ICPMS_LEACH } \\
\mathbf{Z} r \\
\mathrm{mg} / \mathrm{kg}\end{array} \\
<0.004\end{array}$} \\
\hline Blank USGS TCLP & Modified TCLP & \\
\hline Chalmette 1-USGS & Modified TCLP & 0.004 \\
\hline Chalmette1D-USGS & Modified TCLP & 0.02 \\
\hline Chalmette 2-USGS & Modified TCLP & $<0.004$ \\
\hline Chalmette2D-USGS & Modified TCLP & 0.01 \\
\hline BlkUSGS SimBrac & Simulated brackish water & $<0.004$ \\
\hline Chalm 1-USGS & Simulated brackish water & $<0.004$ \\
\hline Chalm 1D-USGS & Simulated brackish water & $<0.004$ \\
\hline Chalm 2-USGS & Simulated brackish water & $<0.004$ \\
\hline Chalm 2D-USGS & Simulated brackish water & $<0.004$ \\
\hline Blank USGS SPLP & Modified SPLP & $<0.004$ \\
\hline Chalme 1-USGS & Modified SPLP & 0.008 \\
\hline Chalme 1D-USGS & Modified SPLP & 0.006 \\
\hline Chalmette 2USGS & Modified SPLP & 0.02 \\
\hline Chalm 2DUSGS & Modified SPLP & 0.02 \\
\hline Blank USGS FLT & USGS FLT & $<0.004$ \\
\hline Chalm 1USGS & USGS FLT & $<0.004$ \\
\hline Chalm1D-USGS & USGS FLT & $<0.004$ \\
\hline Chalm2-USGS & USGS FLT & 0.008 \\
\hline Chalm2D-USGS & USGS FLT & 0.008 \\
\hline Blank NEIC-TCLP & TCLP EPA-NEIC & $<0.004$ \\
\hline Chalmette1-NEIC & TCLP EPA-NEIC & $<0.004$ \\
\hline Chalmette1D-NEIC & TCLP EPA-NEIC-duplicate & 0.004 \\
\hline Chalmette2-NEIC & TCLP EPA-NEIC & 0.004 \\
\hline Chalmette2D-NEIC & TCLP EPA-NEIC-duplicate & $<0.004$ \\
\hline
\end{tabular}


Table 7. Sterol analytical results for samples Chalmette 1 and Chalmette 2, analyzed by the USGS GD Coastal and Marine Geology laboratory, Menlo Park, CA. Contact: Bob Rosenbauer, brosenbauer@usgs.gov.

Target Sterols

Concentration in sample $(\mathrm{ug} / \mathrm{g})^{*}$

Compound

Coprostanol

Epicoprostanol

22-

Dehydrocholesterol

5-beta-cholestan-3-beta-ol

5-beta-cholestan-3-alpha-ol

cholesta-5,22E-dien-3b-ol

Cholesterol

cholest-5-en-3-beta-ol

Desmosterol

5,24-cholestadien-3-beta-ol

Brassicasterol

24-methylcholesta-5,22E-dien-3 beta-ol

24-methylcholesta-5,7,22trien-3

Ergosterol

beta-ol

Stigmasterol

24-ethylcholesta-5,22Edien-3-

beta-ol

beta-Sitosterol

24-ethylcholest-5-en-3-beta-ol

Stigmastastanol

24-ethylcholestan-3-beta-ol

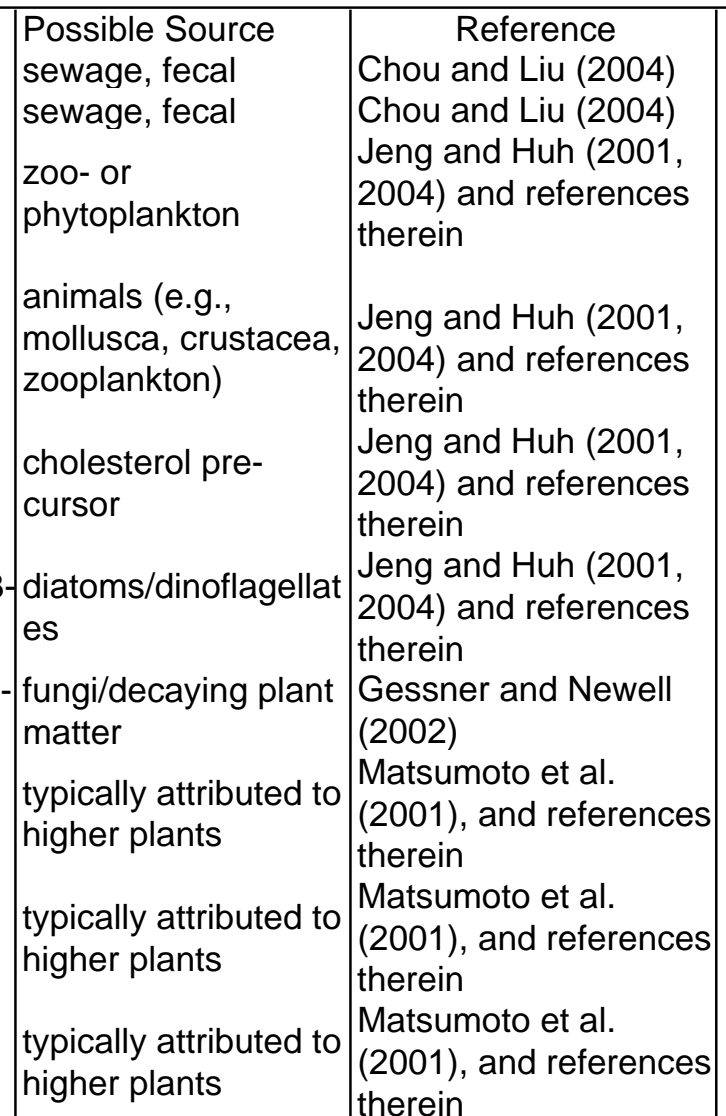

Chalmette

wet $\quad$ dry

wet

0.04

0.04

$\mathrm{dry}^{* *}$

Chalmette 2

0.04

${ }^{*}$ detection limit: $1 \mathrm{ug} / \mathrm{ml}$ injected or $0.01 \mathrm{ug} / \mathrm{g}$ sediment (wet)

**Based on water content: $75 \%$ (Chalmette 1) \& 59.8\% (Chalmette 2) 
Table 8. PAH analytical results from the USGS Coastal and Marine Geology laboratory, Menlo Park, CA. Contact: Bob Rosenbauer, brosenbauer@usgs.gov Target PAH'S

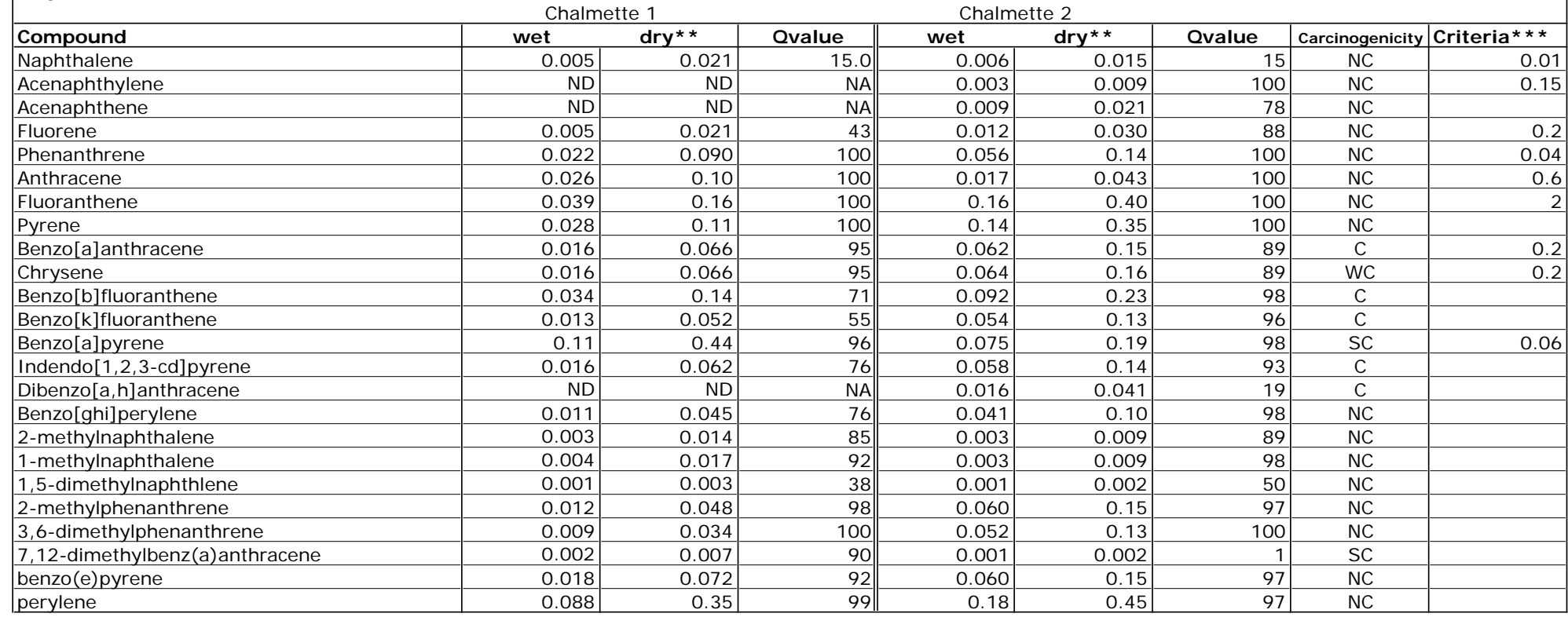

NA: Not Applicable

Qvalue: Qualifier ratio satisfaction

* detection limit: . $01 \mathrm{ug} / \mathrm{ml}$ injected or $0.001 \mathrm{ug} / \mathrm{g}$ sediment (wet)

**Based on water content. $75 \%$ (Chalmette 1$) \& 59.8 \%$ (Chalmette 2)

carcinogenic; SC = strongly carcinogenic. (from Neff, 1979; Rugen and others, 1989; USPHS, 1990)

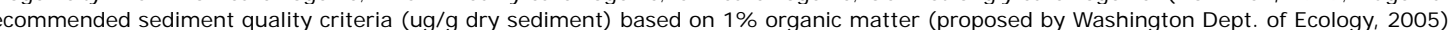




\begin{tabular}{|c|c|c|c|c|}
\hline \multicolumn{5}{|c|}{ ר in sample $(\mathrm{ng} / \mathrm{g})^{*}$} \\
\hline & \multicolumn{2}{|c|}{ Chalmette 1} & \multicolumn{2}{|c|}{ Chalmette 2} \\
\hline compound & wet & dry** & wet & dry** \\
\hline C11 & 62 & 247 & ND & ND \\
\hline $\mathrm{C} 12$ & 61 & 245 & ND & ND \\
\hline C13 & 69 & 277 & ND & ND \\
\hline C14 & 14 & 56 & 12 & 29 \\
\hline C15 & 40 & 162 & 43 & 107 \\
\hline C16 & 68 & 272 & 59 & 146 \\
\hline C17 & 374 & 1496 & 224 & 558 \\
\hline pristane & 124 & 497 & 75 & 186 \\
\hline C18 & 142 & 567 & 81 & 202 \\
\hline phytane & 118 & 472 & 91 & 226 \\
\hline C19 & 171 & 683 & 87 & 217 \\
\hline $\mathrm{C} 20$ & 146 & 584 & 79 & 196 \\
\hline C21 & 180 & 718 & 96 & 238 \\
\hline C22 & 147 & 588 & 67 & 166 \\
\hline C23 & 182 & 729 & 102 & 254 \\
\hline C24 & 157 & 629 & 75 & 186 \\
\hline $\mathrm{C} 25$ & 288 & 1152 & 171 & 424 \\
\hline C26 & 241 & 964 & 65 & 161 \\
\hline C27 & 709 & 2837 & 457 & 1137 \\
\hline C28 & 267 & 1067 & 141 & 351 \\
\hline C29 & 896 & 3586 & 1101 & 2738 \\
\hline C30 & 338 & 1352 & 334 & 832 \\
\hline C31 & 818 & 3273 & 1380 & 3432 \\
\hline C32 & 120 & 478 & 128 & 319 \\
\hline C33 & 363 & 1451 & 501 & 1247 \\
\hline
\end{tabular}

ND: Not Detected

* detection limit: $100 \mathrm{ng} / \mathrm{ml}$ injected or $10 \mathrm{ng} / \mathrm{g}$ sediment (wet) **Based on water content: $75 \%$ (Chalmette 1) \& 59.8\% (Chalmette 2) 
Table 10. Forms of sulfur. Analyst- Cyrus Berry, cberry@usgs.gov

\begin{tabular}{|l|c|c|c|c|}
\hline & $\begin{array}{l}\text { Chalmette 1, } \\
\text { freeze-dried from } \\
\text { wet }\end{array}$ & $\begin{array}{l}\text { Chalmette 1 wet } \\
\text { (recalc. to dry } \\
\text { weight) }\end{array}$ & $\begin{array}{l}\text { Chalmette 2, freeze } \\
\text { dried from wet }\end{array}$ & $\begin{array}{l}\text { Chalmette 2 wet } \\
\text { (recalc. to dry } \\
\text { weight) }\end{array}$ \\
\hline $\begin{array}{l}\text { Acid-volatile } \\
\text { sulfides }\end{array}$ & 0.01 & 0.13 & wt \% & 0.16 \\
\hline $\begin{array}{l}\text { Acid/H2O soluble } \\
\text { sulfates }\end{array}$ & 0.12 & 0.10 & 0.07 & 0.02 \\
\hline Disulfides & 0.96 & 0.97 & 0.40 & 0.36 \\
\hline $\begin{array}{l}\text { Total extractable } \\
\text { sulfur }\end{array}$ & 1.09 & 1.19 & 0.48 & 0.54 \\
\hline
\end{tabular}


Table 11. Net acid production. Contact: Phil Hageman, phageman@usgs.gov. This test was developed to estimate the amount of calcium carbonate that would need to be added to a ton of sulfide-containing mine wastes to neutralize all of the acid generated by oxidation of pyrite and other sulfides (Lapakko and Lawrence, 1993)

Chalmette 1

Chalmette 2

Air-dried in hood from wet $\mathrm{Kg} \mathrm{CaCO} /$ ton $\mathrm{Kg} \mathrm{CaCO} 3 /$ ton

sample 31

0.1

Note: For comparison, results for Chalmette 1 are in the upper quartile of NAP for all mine wastes analyzed by this USGS laboratory. 